\title{
In What Ways Do Synthetic Nucleotides and Natural Base Lesions Alter the Structural Stability of G-Quadruplex Nucleic Acids?
}

\author{
Janos Sagi \\ Rimstone Laboratory, RLI, Carlsbad, CA 92010, USA \\ Correspondence should be addressed to Janos Sagi; jans@rimstonelab.com
}

Received 22 June 2017; Accepted 15 August 2017; Published 18 October 2017

Academic Editor: Tom Marsh

Copyright (C) 2017 Janos Sagi. This is an open access article distributed under the Creative Commons Attribution License, which permits unrestricted use, distribution, and reproduction in any medium, provided the original work is properly cited.

\begin{abstract}
Synthetic analogs of natural nucleotides have long been utilized for structural studies of canonical and noncanonical nucleic acids, including the extensively investigated polymorphic G-quadruplexes (GQs). Dependence on the sequence and nucleotide modifications of the folding landscape of GQs has been reviewed by several recent studies. Here, an overview is compiled on the thermodynamic stability of the modified GQ folds and on how the stereochemical preferences of more than 70 synthetic and natural derivatives of nucleotides substituting for natural ones determine the stability as well as the conformation. Groups of nucleotide analogs only stabilize or only destabilize the GQ, while the majority of analogs alter the GQ stability in both ways. This depends on the preferred syn or anti N-glycosidic linkage of the modified building blocks, the position of substitution, and the folding architecture of the native GQ. Natural base lesions and epigenetic modifications of GQs explored so far also stabilize or destabilize the GQ assemblies. Learning the effect of synthetic nucleotide analogs on the stability of GQs can assist in engineering a required stable GQ topology, and exploring the in vitro action of the single and clustered natural base damage on GQ architectures may provide indications for the cellular events.
\end{abstract}

\section{Introduction}

In the course of the last two decades, substantial worldwide research activity has been devoted to the elucidation of the structural and biochemical properties of the non-B, polymorphic GQ structures, both in vitro and in vivo. Many laboratories used synthetic nucleotide building blocks, which were reviewed by several publications [1-5]. Studies have also focused on such site-specific incorporations of nucleotide analogs that could prompt the modified oligonucleotides to fold into specific GQ topologies, as summarized in [6-8]. In addition to folding topology, the fundamental property of GQ structures is their thermodynamic stability. Multiple aspects of the effect of mutation by natural nucleotides on GQ stability are well known [2-5, 9-11]. Properties of GQs built from peptide nucleic acid (PNA) units $[12,13]$ and of a few other special modifications, such as the (R)1-O-(pyren-1-ylmethyl)glycerol (intercalating nucleic acid, INA) or (R)-1-O-[4-(1-pyrenylethynyl)-phenylmethyl]glycerol (twisted intercalating nucleic acid, TINA) have also been analyzed [4, 14]; therefore, these modifications are not discussed here. Much less has been summed up on the effect of synthetic constituents on the thermal and thermodynamic stability of the modified GQs, which is thus the subject of the present review. Other important current topics are the effect of single and multiple natural base lesions on GQ stability as well as the epigenetic modifications occurring in GQs, the present status of which is also reviewed here up to midsummer 2017.

Single or double-helical guanine-rich (G-rich) natural or synthetic DNA and RNA sequences can, under appropriate physical (cations, cosolvents) or cellular (e.g., negative superhelical stress) conditions, convert into hairpin, triple-helical, and four-stranded GQ structures. A GQ comprises two or more G-tetrads, which are connected by loops of one or more nucleotides. The planar G-tetrads are formed through Hoogsteen-type circular double hydrogen bonds (Figure 1), and the cations are located either in the central cavity of the G-tetrad or in the spaces between the tetrads. The GQ fold 


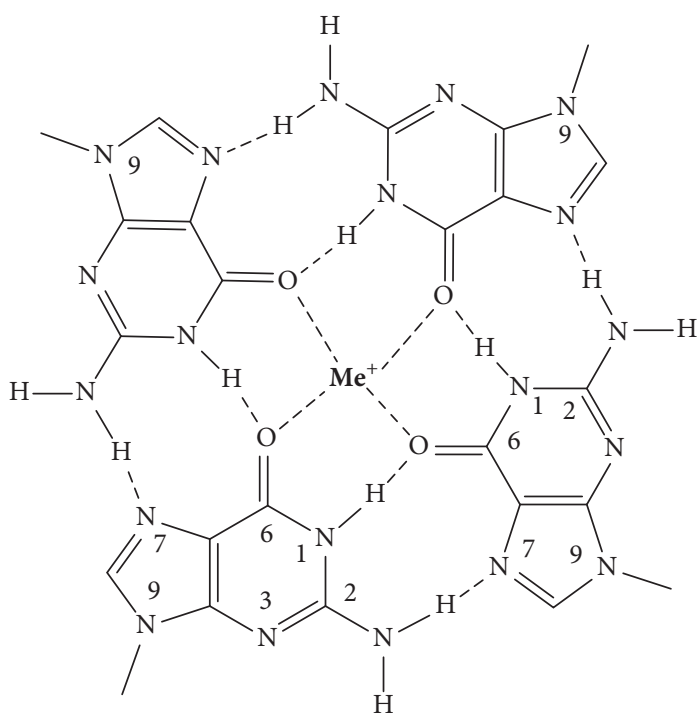

FIGURE 1: Schematic representation of a G-tetrad held together by Hoogsteen-type circular double hydrogen bonds and the metal ion.

is held together by the stacking of G-tetrads and the cation coordination via the $\mathrm{O} 6$ carbonyl atoms of the guanines. In general, the GQs are polymorphic, can be built from single or multiple molecules, and can contain antiparallel, parallel strand, or mixed (hybrid) orientations, and these features result in distinct topological arrangements. Schematic folding architectures of GQs assembling from the 21-nucleotide-long (21-mer) basic human telomere (htel) repeat DNA sequence, htel-21, and of the 15-mer thrombin-binding aptamer (TBA) oligodeoxynucleotide are shown in Figure 2. Characteristic CD spectra of the three main forms of the htel GQs are illustrated in Figure 3. The TBA GQ is the most frequently cited structure in this review due to the dozens of studies performed worldwide using this 15-mer DNA model (e.g., $[3,7,10,15-17])$. The advantage of it is the well-defined stable and single folding topology, which contrasts the polymorphic nature of most other intramolecular GQs known, especially those built from the TTAGGG htel repeat sequences [8]. In physiological $\mathrm{K}^{+}$concentrations, the TBA sequence forms a two-tetrad, chair-type intramolecular antiparallel structure with three edge-type loops, two TT, and one TGT loop (Figure 2). Positions of T4, T9, T13, and G8 are supposed to have rigid positions critical for the stability, and the T3, T7, and T12 are more flexible, not stacked to residues of the G-tetrads [18-20]. TBA GQ inhibits the thrombin-catalyzed fibrin-clot formation with a $K_{i}$ of approximately $120 \mathrm{nM}$ by binding to the thrombin protein with high selectivity. Thrombin is a serine protease that converts soluble fibrinogen to fibrin, thus stimulating platelet aggregation, that is, the clotting process (see references in [21]).

The effect of the synthetic nucleotide analogs is discussed here according to their stabilizing and/or destabilizing action. Those, around a dozen analogs that, under the specific conditions studied, were found to only stabilize or not affect the stability beyond the experimental error of determination of a specific GQ fold, make up one group of the analogs. Majority of the synthetic nucleotides studied so far that either stabilize or destabilize the GQ constitute another, the largest group, and those analogs that only destabilized a GQ belong to the third group of synthetic nucleotide analogs. Similarly, the natural base lesions were also grouped according to their stabilizing or destabilizing effects.

Stability of a GQ structure can be most properly characterized by its thermodynamic parameters communicated by the free energy change value, $\Delta G^{\circ}$, at various temperatures, such as $\Delta G^{\circ}{ }_{37}$, in $\mathrm{kcal} / \mathrm{mol}$. Thermodynamic parameters can be determined by the model-dependent method, such as the "two-state" method using temperature-dependent melting profiles by UV or CD absorption spectroscopy [22, 23], or, more exactly, by the model-independent differential scanning calorimetry (DSC) method [24, 25]. DSC is certainly the more accurate method as it explores the details of the thermal transition contrary to the integrating absorption methods (although not all folding-unfolding transitions fit the twostate requirement, several laboratories neglect it and use the two-state absorption method). Both methods provide the half-way thermal transition temperature, $T_{m}$ or $T_{1 / 2} . T_{1 / 2}$ has been used when the annealing (refolding) is slower than the unfolding, which results in hysteresis of the thermal profile. Since most studies do not publish free energy change values but all publish $T_{m}$ or $T_{1 / 2}$ values, the best way to compare the stabilities of various GQ structures is to use the thermal stability values. $\Delta G^{\circ}$ values will be mentioned herein in selected cases. Figure 3 shows typical thermal melting profiles as determined by UV absorption spectroscopy at $296 \mathrm{~nm}$ [26].

\section{Synthetic Nucleotide Analogs That Stabilized or Barely Impacted the Stability of a GQ}

Few synthetic analogs of natural nucleotides are currently known, which only stabilize a GQ independently of their syn or anti isomeric conformation and/or the position of the nucleotide they replaced in the potential GQ-forming oligonucleotide. Similarly, a limited number of analogs have so far been described that do not affect the thermodynamic stability of GQs beyond the experimental error of determination. It is important to note that these effects were observed in a given GQ system; the nucleotide analogs may induce the opposite effect in other, so far not-studied GQs.

N2-Guanine Derivatives. The C2-amino group of dG (Figure $4, \mathbf{1}$ ) is one of two positions of $\mathrm{dG}$ whose substitutions do not interfere with the circular double H-bonding of a Gtetrad $[27,28]$. Substitution of N2 does not change the glycosidic torsion angle of the anti dG. The 2-amino group of guanine substituted with hydrophobic groups can enhance the thermodynamic stability of a GQ via disrupting the water sphere around the core. Water depletion is known to stabilize a GQ (and destabilize duplex DNAs) [29, 30]. The TGGGAG oligonucleotide DNA modified at the $3^{\prime}-$ and $5^{\prime}$ ends with 3,4-dibenzyloxybenzyl groups (R-95288, Hotoda oligonucleotide), which forms a parallel tetramolecular GQ (Figure 2, 6), has anti-HIV-1 activity through binding to 

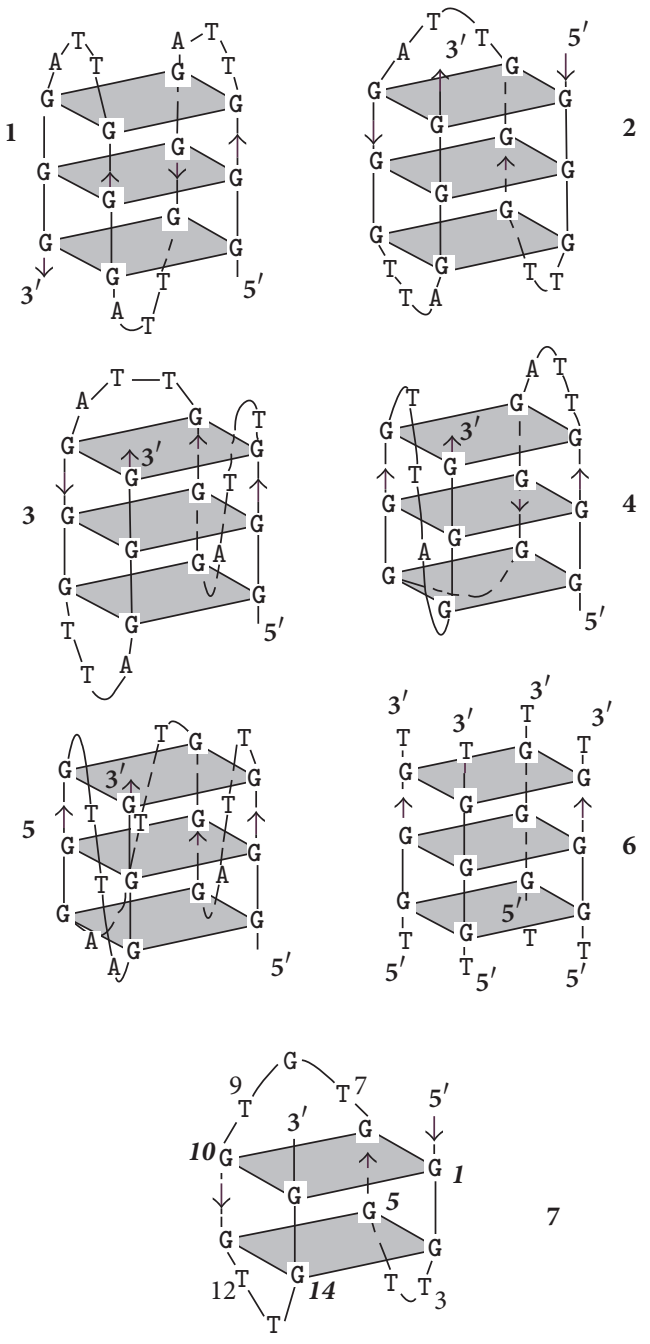

$5^{\prime}$ - GGTTGGTGT GGTTGG

FIGURE 2: Folding arrangements of the most often cited GQ structures. The intramolecular GQ architectures adopted by the 21-mer htel-21 $\mathrm{G}_{3}\left(\mathrm{TTAG}_{3}\right)_{3}$ oligodeoxynucleotide under various solution conditions, topologies 1-5. The basket-type antiparallel with $5^{\prime}$-to-3' edgewisediagonal-edgewise loops, forming in $\mathrm{Na}^{+}$(topology 1); the chair-type antiparallel with three edgewise loops (topology 2); the (3+1 strands) hybrid-1 with propeller-like, edgewise, and edgewise loops (topology 3 ); the hybrid-2 with two edgewise loops followed by a propeller-type loop (topology 4), both hybrids forming in $\mathrm{K}^{+}$solution; the parallel topology with all three loops in propeller-like configuration, as found in crystals containing $\mathrm{K}^{+}$ions (topology 5); tetramolecular GQ assembling from four TGGGT DNA chains, $\left[\mathrm{TG}_{3} \mathrm{~T}_{4}\right.$ (topology 6 ). Bottom panel: the two-tetrad, chair-type intramolecular antiparallel topology, folding from the 15-mer TBA oligodeoxynucleotide sequence shown below the topology. The syn $\mathrm{dG}$ nucleotides are indicated with underlined letters in italics. For references, see, for example, [8]. Shaded squares represent the G-tetrads displayed in Figure 1.

gp120 [31]. dGs of this oligodeoxynucleotide had been substituted with $\mathrm{N} 2$-methyl-2'-deoxyguanosine $\left(\mathrm{m}^{2} \mathrm{dG}\right)$. The $\mathrm{m}^{2} \mathrm{dG}$ modifications enhanced the stability of the parent GQ and the modified oligonucleotides showed a 2-fold higher anti-HIV activity than the R-95288. The GQs formed from T. $\underline{m}^{2}$ GGGAG and TGG $\underline{m}^{2}$ GAG had higher $T_{m}$ values, $70^{\circ} \mathrm{C}$, than that assembled from TGGGAG, $T_{m}$ of $60^{\circ} \mathrm{C}$. On the other hand, the $3^{\prime}$-terminal substitution with $\mathrm{m}^{2} \mathrm{dG}$ had no effect on the GQ stability due to the poor stacking of the terminal base. The increased stabilities were explained by improved base stacking originating from the methylation of $\mathrm{NH}_{2}$ of $\mathrm{G} 2$. The GQ of $\mathrm{T}\left[\mathbf{m}^{2} \mathbf{G}\right]_{3} \mathrm{AG}$ with three contiguous $\mathrm{m}^{2} \mathrm{dG}$ residues had the highest $T_{m}$, which was over $80^{\circ} \mathrm{C}$. The alkylation of $\mathrm{N} 2 \mathrm{G}$ seems to be a promising way to improve the stability of parallel GQs, as long as the modification does not sterically hinder the formation of the GQs [32]. Substitution of $\mathrm{N} 2$ of $\mathrm{G}$ was also mentioned in connection with the TBA GQ (Figure 2, 7), however, only in connection with the thrombin-binding activity: attaching of a benzyl group to N2 of the anti N-glycosidic dGs (Figure 4, 1) of positions G6 and G11 and a 1-naphthylmethyl group into the N2 of G6 of the TBA increased the thrombin inhibitory activity. The 1naphthylmethyl group substitution at the N2 of G6 showed about a $60 \%$ increase in activity both in vitro and in vivo. 


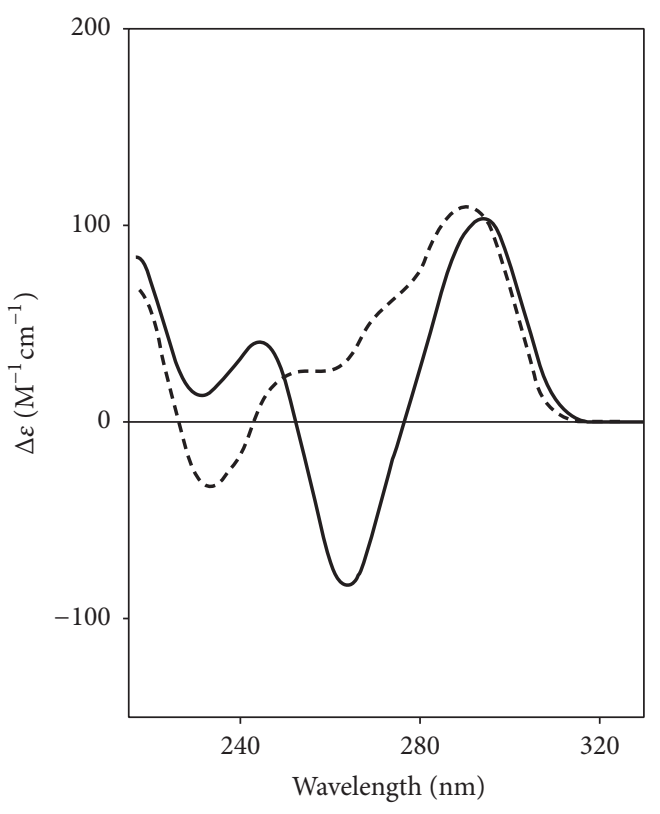

(a)

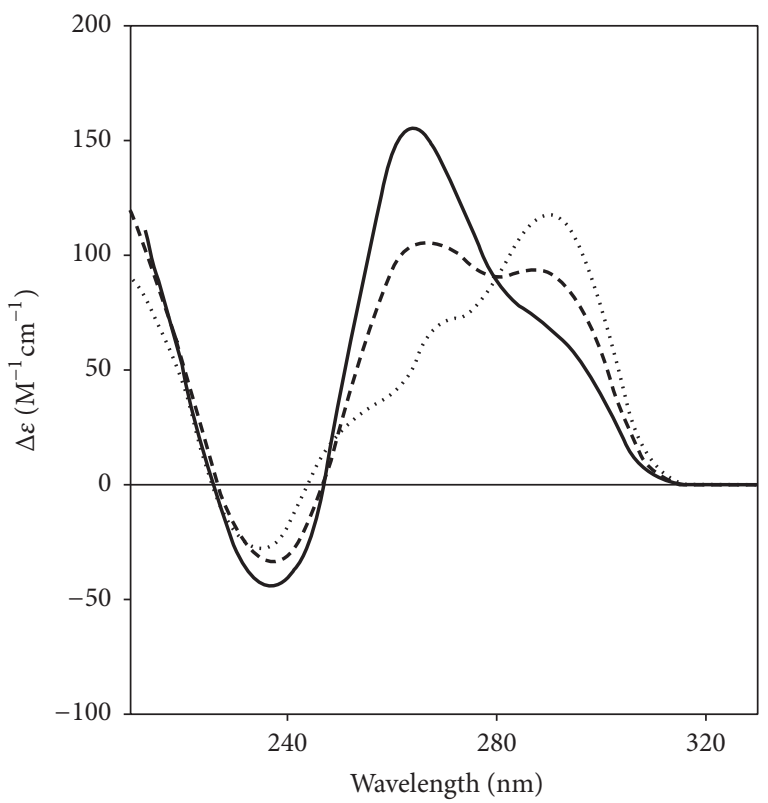

(b)

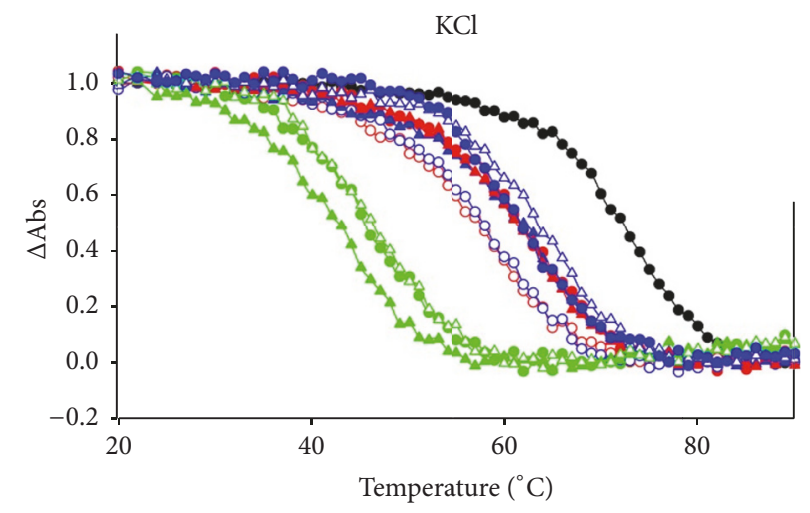

(c)

FIGURE 3: CD spectra of different GQ topologies and a series of thermal unfolding profiles. (a) shows the CD spectra of the antiparallel basket architecture of htel-21 GQ recorded in $100 \mathrm{mM} \mathrm{Na}^{+}$solution and characterized by positive Cotton effects near $295 \mathrm{~nm}$ and $240 \mathrm{~nm}$ and a negative one around $260 \mathrm{~nm}$ (solid line), as well as the polymorphic form adopted by the same oligodeoxynucleotide in $100 \mathrm{mM} \mathrm{K}^{+}$, which is a mixture of $\mathrm{K}^{+}$-stabilized antiparallel and the hybrid forms, with the positive ellipticity near $290 \mathrm{~nm}$, a strong shoulder around $270 \mathrm{~nm}$, and a negative one close to $240 \mathrm{~nm}$ (broken line). (b) displays, for comparison, the CD spectra of the same $\mathrm{K}^{+}$-stabilized mixture of the htel-21 GQ (dotted line) and the mixture of just the hybrid forms with two positive peaks of close to equal heights at $290 \mathrm{and} 260 \mathrm{~nm}$ (dashed line), all determined at low (50-100 $\mu \mathrm{M})$ DNA strand concentrations, as well as the majority parallel forms adopted at/above $2 \mathrm{mM}$ of the strands (solid line) (see references in [8]). (c) illustrates normalized thermal unfolding profiles of 8-oxoguanine containing htel-21 GQs [26].

Substitutions on N2 of other G residues of the sequence had a little effect or decreased the activity [33]. The latest example for stabilization of GQ structures by N2-dG substitutions comes from using htel GQs. Lech and Phan [34] reported on the incorporation of N2-methyl, N2-hexylamino, and the N2-benzyl derivatives of $\mathrm{dG}$ into an htel-24 DNA GQ, the TT(htel-21)A. The substitutions stabilized the G-quadruplex by 2 to $13^{\circ} \mathrm{C}$ per modification.

Isoguanine ( $i G$ ). 2-Oxo-6-aminopurine, isoguanine (Figure 4, 2), has been thoroughly studied in GQ models, first by Seela and coworkers [35] with $\mathrm{T}_{4} \mathbf{i G}_{4} \mathrm{~T}_{4}$ that formed a tetramolecular parallel GQ, which contained iG-tetrads held together by circular double hydrogen bonds. The $\left[\mathrm{T}_{4} \underline{\mathbf{i G}}_{4} \mathrm{~T}_{4}\right]_{4}$ proved to be a more stable structure than the parent $\left[\mathrm{T}_{4} \mathrm{G}_{4} \mathrm{~T}_{4}\right]_{4}$ in

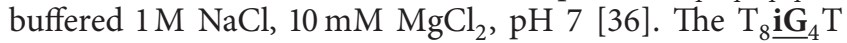
also formed parallel GQ with iG-tetrads [37]. The iG formed not only tetrads but also pentads, giving rise to pentaplexes, such as $\left[\mathrm{T}_{4} \underline{\mathbf{i}}_{4} \mathrm{~T}\right]_{5}$, and the pentad planes were held together, as the tetrads, by circular double H-bonds [38-40]. The iGpentaplexes were found to bind and activate the Fe(III)heme complex towards peroxidase activity, comparably to GQs and contrary to iGQs [41]. In the intramolecular GQ of TBA, a single iG residue was found to enhance the binding of GQ to the human R-thrombin, as compared to the parent GQ aptamer. The most active aptamer was built 


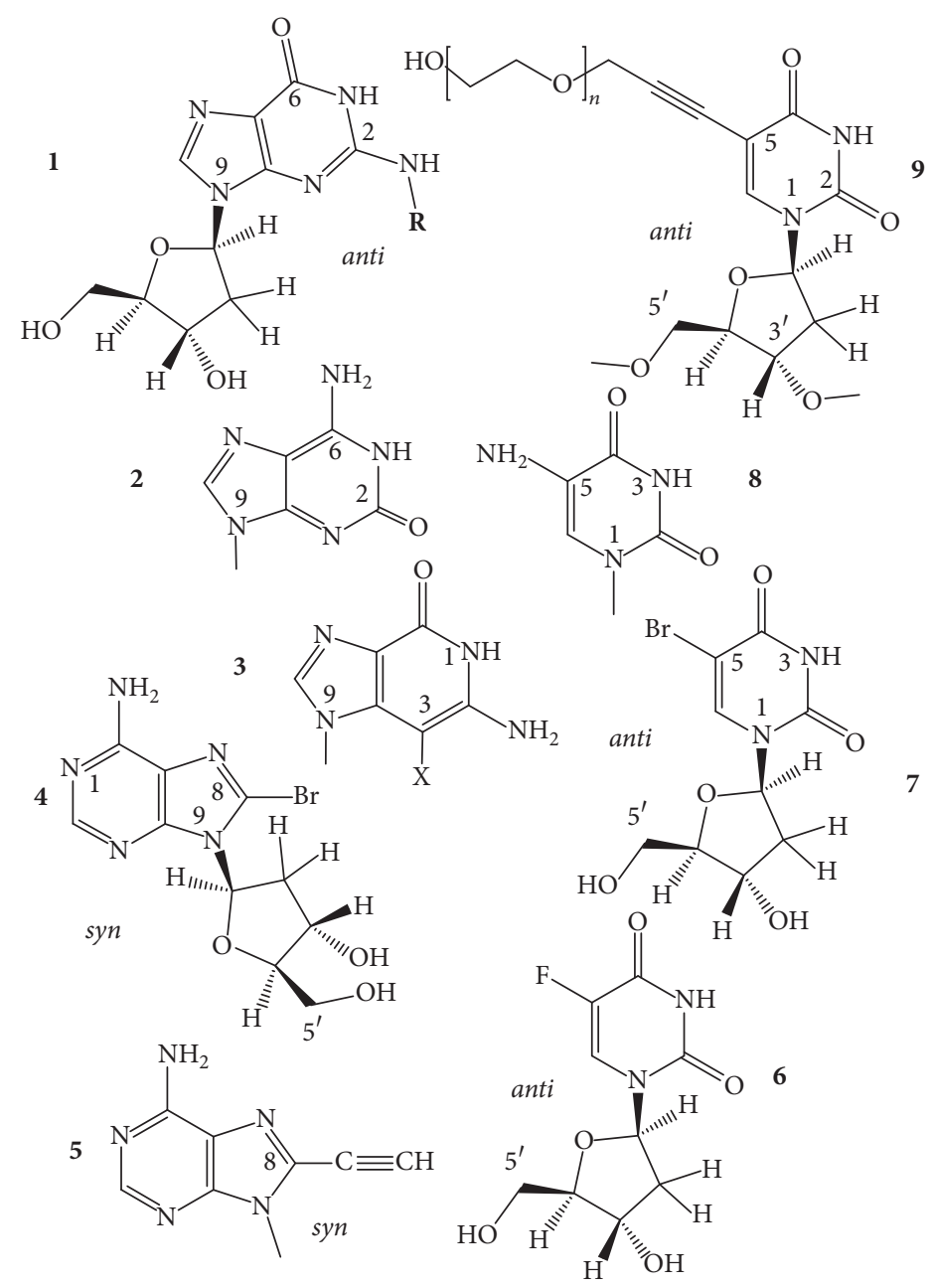

FIGURE 4: Structures of the anti N2-substituted-dG (1); isoguanine (2); 3-halo-3-deazaguanine (3); syn 8-bromo-dA (4); 8-propynyladenine (5); anti 5-fluoro-dU (6); anti 5-bromo-dU (7); 5-aminouracil (8); and oligo(ethylene glycol) substituted anti 5-propynyl-dU (9).

from GGTTGGTiGTGGTTGG, and its approximate binding enhancement was 2 -fold over the unmodified aptamer. The authors supposed that iG-GQs tended to form and denature more easily than the corresponding structures bearing G. Whether this would mean increased or decreased thermodynamic stability is not known [21].

3-Deazaguanine $\left(c^{3} G\right)$. Quantum chemical methods-based models showed that 3-halo-3-deazaguanines (Figure 4, 3) replacing guanines in two- or three-tetrad GQs result in substantial energy gain leading to elevated stability for the GQs, which mainly originates from increased $\pi-\pi$ stacking interactions [42].

8-Bromo-, 8-Methyl-, and 8-Propynyladenines. In the tetramolecular GQ-forming TAGGGT and AGGGT oligodeoxynucleotides, the adenines have been replaced by 8 -bromadenine ( $\mathrm{br}^{8} \mathrm{dA}$ ), 8-propynyladenine (py ${ }^{8} \mathrm{dA}$ ) (Figure 4, 4, 5) [43-45], and 8-methyladenine $\left(\mathrm{m}^{8} \mathrm{~A}\right)[46,47]$. All these A-modified oligonucleotides formed the same parallel-type tetramolecular GQs as the two unmodified oligonucleotides did. While $\mathrm{py}^{8} \mathrm{~A}$ decreased the stability of duplex DNAs, it markedly elevated the thermal stability of the tetramolecular structures, assumingly, due to a prevalent glycosidic syn conformation $[43,45]$. br $^{8} \mathrm{~A}$ also increased the stability of the parent structures [44]. The GQ analogs of TAGGGT did not contain T, A, or 8-substituted-A tetrads, whereas the GQ of AGGGT formed an A-tetrad at the $5^{\prime}$-end. An $\mathrm{m}^{8} \mathrm{dA}$ tetrad was also formed; it was an all-syn $\mathrm{m}^{8} \mathrm{dA}$ tetrad that had only a minor effect on $T_{m}$ of the parent structure.

5-Fluorouracil $\left(f l^{5} \mathrm{U}\right)$. Thymines of the TBA oligodeoxynucleotide were replaced, one by one, with 5 -fluoro- $2^{\prime}$ deoxyuridines (Figure 4, 6) by Virgilio et al. [48]. The 5-fluoro substitution for the 5-methyl group elevated the thermal stability of the GQ in each of the six cases studied, mostly by $1-3^{\circ} \mathrm{C}$; however, in positions 4 and $13, \mathrm{fl}^{5} \mathrm{U}$ enhanced the stability by 11 and $10^{\circ} \mathrm{C}$, respectively. These two GQs also showed improved anticoagulant activity. Thymine bases at the $\mathrm{T} 4$ and T13 loop positions stack to the tetrad residues [18-20]. The high $T_{m}$ values may indicate that the fluoro atom enhanced the strength of stacking. Thymines in other loop positions do 

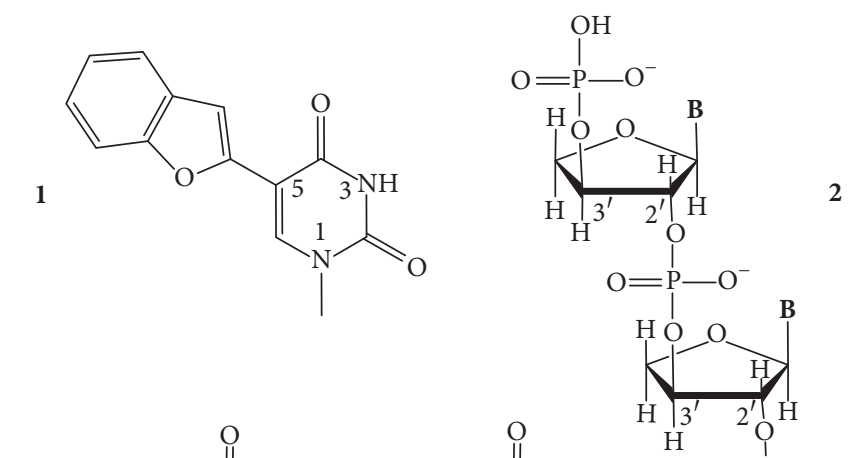<smiles>[R4]C(Cn1c2c(c(=O)[nH]c1=O)CCC2)[C@H](O)CO</smiles><smiles>O=c1[nH]c(=O)n(C[C@H](O)CO)c2c1CCC2</smiles>

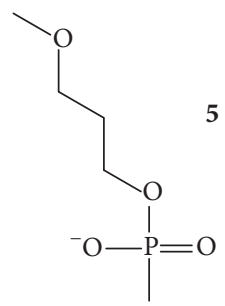

Figure 5: Structures of 5-(benzofuran-2-yl)uracil (1); threose dinucleotide (2); R- (3) and S-isomer (4) of acyclic-uracil derivatives and the n-propyl spacer (5).

not interact with the GQ core. The modified GQs adopted the same type of antiparallel topology as the wild-type did, based on the CD spectra.

5-Bromo-, 5-Amino-, and 5-Hydroxyuracils. Tetrad thymines had been replaced by the synthetic 5-bromouracil $\left(b r^{5} \mathrm{U}\right)$ and 5-aminouracil $\left(\mathrm{n}^{5} \mathrm{U}\right)$ (Figure $\left.4,7,8\right)$ that substituted for the central T of TGGTGGT. The oligonucleotides modified by these dU-analogs formed tetramolecular parallel GQs, as the unmodified did, with all residues having anti glycosidic conformation. Order of thermal stability was $n^{5} U>$ br $^{5} U>T$ containing GQs. The [TGG $\left.{ }^{5}{ }^{5} G G T\right]_{4}$ GQ showed a biphasic melting profile, which was explained by noncooperative melting. The $\mathrm{n}^{5} \mathrm{dU}$ tetrad was observed having circular double $\mathrm{H}-$ bonds and this provided higher stability than T or br ${ }^{5} U$ [49]. Using Density Functional Theory calculations and ESI-MS measurements, Paragi et al. [50] hypothesized that 5-aminoand especially 5-hydroxyuracils can form full pyrimidine and also mixed tetrads with guanine or xanthine, where tetrads have similar energy and cover almost exactly the same area as the full guanine or xanthine tetrads.

5-Propynyluracil and Its OEG and PEG Derivatives. Based on their previous findings [51] Tateishi-Karimata and coworkers [52] described in a recent paper the stabilizing effect of 5propynyl- $2^{\prime}$-deoxyuridine on the TBA GQ when it was substituted for T4 and T7 of the loops. T base at position 4 stacks to the G-tetrad, while the T7 does not [18-20]. The propynyluracil substitution of T4 enhanced the stability of the native TBA GQ $\left(T_{m}: 50.7^{\circ} \mathrm{C}, \Delta G^{\circ}{ }_{25}:-3.5 \mathrm{kcal} / \mathrm{mol}\right)$ up to $61.7^{\circ} \mathrm{C}$ and $-5.4 \mathrm{kcal} / \mathrm{mol}$, whereas the nonstacking did not change it: $50.9^{\circ} \mathrm{C}$ and $-3.6 \mathrm{kcal} / \mathrm{mol}$, in buffered $100 \mathrm{mM} \mathrm{KCl}$. The propynyluracil was further derivatized with ethylene glycols (Figure 4, 9). Oligo- and polyethylene glycols (OEG and PEG) are used as crowding agents in vitro to mimic the intracellular environments. Both destabilize the dsDNA but stabilize the GQs [30]. Various lengths and numbers of ethylene glycols were attached to the 5-(1-propynyl)-tethered dUs and substituted for the T4, T7, and T13 of the TBA GQ to learn whether these glycols interact with the GQ. Based on thermodynamic and NMR spectral analyses, they have found that the glycols do interact with the bases of the GQ, and the octaethylene (OEG8) and longer-chain glycols elevated the thermodynamic stability of the parent GQ beyond the stability increase induced by the propynyl tether alone. For instance, the $T_{m}$ and $\Delta G^{\circ}{ }_{25}$ values of $50.7^{\circ} \mathrm{C}$ and $-3.5 \mathrm{kcal} / \mathrm{mol}$ for the TBA GQ were increased to $64.3^{\circ} \mathrm{C}$ and $-7.1 \mathrm{kcal} / \mathrm{mol}$ and $55.2^{\circ} \mathrm{C}$ and $-4.4 \mathrm{kcal} / \mathrm{mol}$ for the propynyl-OEG(8)-dU at positions 4 and 7, respectively. The results suggested that PEG molecules interact with tetrad and loop bases via $\mathrm{CH}-\pi$ and lone pair- $\pi$ interactions [52].

5-(Benzofuran-2-yl)uracil. Tanpure and Srivatsan [53] reported that the 5-(benzofuran-2-yl)uracil (Figure 5, 1) can be used to monitor the formation of GQs from htel DNA and 
RNA sequences. The uracil probe exhibited 4- to 9-fold fluorescence intensity enhancements in the GQ compared with its duplex structure. The effect depended on the GQ topology and on the position of the modification. The probe minimally perturbed the GQ topology and stability and could distinguish the antiparallel, hybrid, and parallel topologies of DNA and RNA GQs from the corresponding duplexes. UV thermal melting profiles showed that the unmodified and the modified GQs had similar $T_{m}$ values, within $\leq 2^{\circ} \mathrm{C}$, even in different ionic conditions.

Threose Nucleotide (TNA). The sugar analog $\alpha$-L-threofuranosyl nucleotide (TNA for threose nucleic acid) (Figure 5, 2) has been examined if the oligonucleotides containing this shorter internucleotide building unit can also form GQs [54]. Despite the modified backbone repeat unit, which is one atom shorter than that found in DNA and RNA, TNA could selfassemble into stable tetramolecular GQ structures that are similar in thermal stability to the $2^{\prime}$-deoxyribose-containing $\left[\mathrm{TG}_{4} \mathrm{~T}\right]_{4}$. Unlike DNA, the TNA GQs formed equally well in either $\mathrm{Na}^{+}$or $\mathrm{K}^{+}$ions [54].

Acyclic-Uracil Derivatives. The R-stereoisomer of an acyclic uracil containing a cyclopentane ring fused at positions 5 and 6 of the uracil ring (R-c, Figure 5,3 ) had been introduced by Borbone et al. [55] into position T7 or T12 (TGT and the TT loops) of the TBA oligodeoxynucleotide. The resulting two aptamers folded into the typical antiparallel chair-like GQ structures formed also by the native TBA in $\mathrm{K}^{+}$buffer (Figure 2). The acyclic residue increased the thermal stability of the resulting GQs with respect to the TBA GQ, by $1^{\circ} \mathrm{C}$ and $4^{\circ} \mathrm{C}$, respectively. Anticoagulant activity of the TBA-T7 was more potent than the TBA's in prolonging clotting time [55]. Later, the research group incorporated both the $\mathrm{S}$ and the $\mathrm{R}$ stereoisomers (S-c and R-c; Figure 5, 3, 4) into the same TBA sequence into positions 3,7 , and 12 . Positions 3 and 12 are located in the two TT loops, and position 7 is located in the TGT loop. At these locations, both isomers of the acyclic-T derivative enhanced the thermal stability of the resulting GQs and all adopted the same antiparallel topology as the parent TBA GQ did. $T_{m}$ of the unmodified GQ, $50^{\circ} \mathrm{C}$, was raised to $54-55^{\circ} \mathrm{C}$ by the R-derivatives and to $51-56^{\circ} \mathrm{C}$ by the $\mathrm{S}$ derivatives [56] (it is not known how these derivatives would influence the thermal stability in the other three $\mathrm{T}$ positions of the loops).

n-Propyl Spacer. A three-carbon spacer (Figure 5, 5) replaced the base-sugar residues of T3, T7, and T12 loop positions of the TBA GQ. The flexible abasic sites increased $T_{m}$ of the unmodified TBA GQ $\left(49^{\circ} \mathrm{C}\right)$ by $6^{\circ} \mathrm{C}$ at each of the three positions and did not alter the antiparallel chair folding topology of the unmodified GQ. The modifications improved the thrombin clotting time [57].

Other Stabilizing Analogs. A novel fluorescent TBA analog has been created by conjugation with a dansyl probe at the $3^{\prime}$-end and a $\beta$-cyclodextrin residue at the $5^{\prime}$-end. The bisconjugated TBA analog could fold into the chair-like, antiparallel GQ structure in $\mathrm{K}^{+}$, typical of the native TBA GQ, and retained its thrombin-binding properties as well. The terminal appendages only marginally affected the conformational features of the TBA GQ. Folding into GQ was associated with a net fluorescence enhancement due to encapsulation of the $3^{\prime}$-dansyl group into the apolar cavity of the $\beta$-cyclodextrin at the $5^{\prime}$-end. This novel analog of the TBA GQ demonstrated its potential as a diagnostic tool for thrombin recognition and also provided a useful basis for the design of suitable aptamerbased devices for theranostic applications, that is, allowing simultaneous detection and inhibition or modulation of the thrombin activity. The terminal double conjugation increased $T_{m}$ of the native TBA by about $10^{\circ} \mathrm{C}[58]$.

The short $\mathrm{TG}_{4} \mathrm{~T}$ oligodeoxynucleotide forms a stable tetramolecular parallel-stranded GQ in $\mathrm{K}^{+}$solution. The $3^{\prime}$ and/or the $5^{\prime}$-ends of four $\mathrm{TG}_{4} \mathrm{~T}$ molecules have been linked together by a non-nucleotide-type, tetra-end-linker (TEL) by Oliviero et al. [59]. The TEL- $\left[\mathrm{TG}_{4} \mathrm{~T}\right]_{4}$ architectures were able to form parallel GQs regardless of the TEL size and the structural orientation of the oligonucleotide branches. The TEL- $\left[\mathrm{TG}_{4} \mathrm{~T}\right]_{4}$ structures that had longer TEL were more thermostable than those with the shorter TEL.

A perylene group, a five-membered fused aromatic structure, has been linked to the nonbonded oxygen of the phosphodiester linkage between dG-dG, dG-T, and T$\mathrm{T}$ dinucleotides, and a single perylene-modified dimer was introduced in the TBA and the 20 -mer (TGGGT) ${ }_{4}$ oligonucleotides to develop fluorescence anisotropy GQ probes. Single modifications showed little effect on GQ conformation and the thermostability increased in both $\mathrm{K}^{+}$and $\mathrm{Pb}^{++}$if the perylene moiety was not located between the dG stacks. With some modified sequences, $T_{m}$ of the resulting GQ increased by $32^{\circ} \mathrm{C}$. In most cases, the GQs were more stable in $\mathrm{Pb}^{++}$than in $\mathrm{K}^{+}$with $T_{m}$ increasing up to $26^{\circ} \mathrm{C}$. The large stabilization was probably due to the interaction of perylene with the G-quartet plane and a more compact structure was induced by $\mathrm{Pb}^{++}$. CD spectra of the (TGGGT) $)_{4} \mathrm{GQ}$ and the modified analogs showed parallel topology in the presence of $\mathrm{K}^{+}$with a positive peak near $265 \mathrm{~nm}$ and a negative one near $240 \mathrm{~nm}$. The TBA and its analogs displayed positive peaks near 245 and $295 \mathrm{~nm}$ and a negative one near $265 \mathrm{~nm}$, typical of the antiparallel GQ structures. In the presence of $\mathrm{Pb}^{++}$, the positive peaks of $265 \mathrm{~nm}$ and $295 \mathrm{~nm}$ of the structures were red-shifted, indicating the $\mathrm{Pb}^{++}$-stabilized antiparallel GQs in both scaffolds [60].

Non-nucleoside-type linkers, such as propanediol, octanediol, or hexaethylene-glycol, that replaced the whole TTA loops connecting the four GGG tracts of an htel GQ induced the formation of parallel GQs, like the singlenucleotide loops do, as Risitano and Fox have reported [61]. A special feature of these loop-replaced structures was that $T_{m}$ of all of these was above $37^{\circ} \mathrm{C}$ in the presence of $10 \mathrm{mM} \mathrm{Li}^{+}$ alone, without added $\mathrm{K}^{+}$, in contrast to any wild-type htel GQ. In $40 \mathrm{mM} \mathrm{K}^{+}$, all loop-replaced htel analogs melted at higher temperatures than the wild-type TTA loop-containing $5^{\prime} \mathrm{F}_{-} \mathrm{G}_{3} \mathrm{TTAG}_{3} \mathrm{TTAG}_{3} \mathrm{TTAG}_{3}$-Q GQ $\operatorname{did}\left(T_{m} 69^{\circ} \mathrm{C}\right)$, where $\mathrm{F}$ and $\mathrm{Q}$ stand for the fluorophore and the quencher molecules, respectively. 
<smiles>[13CH3][C@H]1O[C@@H](n2c(Br)nc3c(=O)[nH]c(N)nc32)[C@H](O)[C@@H](O)[C@@H]1CO</smiles><smiles>Cc1nc2c(=O)[nH]c(N)nc2n1C</smiles><smiles>COc1nc2c(=O)[nH]c(N)nc2n1C</smiles><smiles>Cn1c(N)nc2c(=O)[nH]c(N)nc21</smiles>
$\operatorname{syn}$

Figure 6: Structures of anti 2' -deoxyguanosine (1); syn 8-bromo-dG (2); 8-methylguanine (3); 8-O-methylguanine (4); 8-aminoguanine (5); 8-propynylguanine (6).

\section{Stabilization or Destabilization of a GQ by the Same Synthetic Nucleotide Analog}

Depending on the folding topology, GQs contain nucleosides of anti and syn $\mathrm{N}$-glycosidic linkages, which connect the base and sugar moieties. In most cases, the parallel GQs of any molecularity contain all anti nucleosides, and the antiparallel and hybrid architectures (Figure 2) comprise both types of natural nucleosides. Although the thermodynamic variance between the two conformation states is small, in the range of $1-2 \mathrm{kcal} / \mathrm{mol}$, forcing a nucleoside from syn to anti or vice versa does affect the thermodynamic stability of a GQ and generally leads to destabilization. In some cases, it also induces topological rearrangement of the GQ. In this way, all 8 -substituted $2^{\prime}$-deoxyguanosines whose thermodynamically preferred glycosidic conformation is syn, such as the 8bromo-dG and 8-methyl-dG, if incorporated into syn dG positions generally enhance the thermodynamic stability of the native GQ, whereas if incorporated into anti $\mathrm{dG}$ positions they generally reduce the stability. Similarly, if the anti stabilized sugar analogs substitute for syn $\mathrm{dG}$ positions, these not only destabilize the structure but can also alter the folding topology. For instance, an antiparallel fold, such as that of the monomolecular TBA GQ, transforms into a nonmonomolecular parallel fold on the effect of such substitutions. Another factor in the stabilization-destabilization is the sequence position within the potential GQ-forming oligonucleotide. The same nucleotide analog, independently of the syn/anti isomerism, can increase the stability at a given position and decrease it in another location. This alternate effect depends on the role of the substituted position in the stabilization of the folded native structure.

\subsection{Incorporation of Base Derivatives}

3.1.1. Purine Analogs. In GQs, the position C8 and also one hydrogen atom of $\mathrm{NH}_{2}$ of $\mathrm{C} 2$ of guanine are the only suitable sites for modification that may not cause major deformation of the folding topology. Substituents of these positions point towards the grooves, towards the solvent, away from the Hoogsteen-type circular double H-bonding structure, and away from the central cavity of the stacked G-tetrads. C8 of guanine has been the most frequently modified position. The energetically preferred $\mathrm{N}$-glycosidic torsion angle of $2^{\prime}$-deoxyguanosine $(\mathrm{dG})$ is in the anti range (Figure $6, \mathbf{1}$ ), whereas bulky atoms, like the halogens, the oxygen, and groups that are large enough to sterically interfere with the $5^{\prime}$ $\mathrm{OH}$ of the sugar moiety, turn the glycosidic bond into the synregion (Figure 6, 2-6) [62-64]. The difference between these conformations has been estimated to be $\sim 1-2 \mathrm{kcal} / \mathrm{mol}$ [33]. When the 8-substituted-dG replaces an originally syn $\mathrm{dG}$, it can generally stabilize the scaffold for which the best example is 8 -bromo-dG.

8-Bromo-2' -deoxyguanosine ( $\left.b r^{8} d G\right)$. Dias et al. [64] described in 1994 that incorporation of $\mathrm{br}^{8} \mathrm{dG}$ into the 20 -mer $\left(\mathrm{T}_{3} \mathrm{G}_{2}\right)_{4}$, which folded into a monomolecular antiparallel chair-type GQ (Figure 2, 2), stabilized the $\left(\mathrm{T}_{3} \mathrm{G}_{2}\right)_{4}$ by $5-6^{\circ} \mathrm{C}$ in the $T_{m}$ value when syn dGs were switched to $\mathrm{br}^{8} \mathrm{dGs}$. Free energy 
difference $\left(\Delta \Delta G^{\circ}{ }_{25}\right)$ between the parent and the syn $\mathrm{br}^{8} \mathrm{dG}$ modified GQs was $-1 \mathrm{kcal} / \mathrm{mol}$. The $\mathrm{br}^{8} \mathrm{dG}$-induced destabilization also came first from this study. The $\left(\mathrm{T}_{3} \mathrm{G}_{2}\right)_{4}$ GQ was destabilized by $3-6^{\circ} \mathrm{C}$ in its $T_{m}$ when the anti $\mathrm{dG}$ nucleotides were replaced by $b^{8} \mathrm{dG}$. Free energy difference between the parent and the eight syn- and anti substituted $\mathrm{br}^{8} \mathrm{dG}$ modified GQs was +1 or $-1 \mathrm{kcal} / \mathrm{mol}$. Xu et al. [65] found in 2006 that $\mathrm{br}^{8} \mathrm{dG}$ stabilized the GQ fold of the 22-mer human telomere sequence $\mathrm{A}\left(\right.$ htel-21) or $\mathrm{AG}_{3}\left(\mathrm{TTAG}_{3}\right)_{3}$ in $\mathrm{K}^{+}$ solution when $\mathrm{br}^{8} \mathrm{dG}$ was incorporated in place of a syn dG position of the 3-tetrad monomolecular GQ. The enhanced thermodynamic stability was supposed to be the driving force for the originally polymorphic folds [8] (Figure 2, 1-4) to convert into a more stable single form. As a result, $\mathrm{br}^{8} \mathrm{dG}$ has since been frequently used to stabilize particular DNA and RNA GQ conformations for structural studies carried out with CD, UV, NMR spectroscopy, and other techniques [66-93]. In certain cases, $\mathrm{br}^{8} \mathrm{dG}$ could stabilize a GQ also in anti $\mathrm{G}$ positions. Aviñó et al. [94] substituted the TBA GQ (Figure 2,7) with $\mathrm{br}^{8} \mathrm{G}$, which stabilized the GQ when substituted for the syn position $\mathrm{G} 5$, by $7.3^{\circ} \mathrm{C}$, and interestingly also by the double substitutions for G2, G11, by $3.6^{\circ} \mathrm{C}$, which were both anti positions. The TBA GQ remained antiparallel. The analog destabilized the GQ in the anti position G2 by $2.5^{\circ} \mathrm{C}$ and also by double substitutions of the anti positions $\mathrm{G} 2$ and $\mathrm{G} 6$ by $8.8^{\circ} \mathrm{C}$ [94].

A remarkable example of using br $^{8} \mathrm{dG}$ for structure studies was the stabilization of individual topologies in telomeric GQs. Attaching flanking nucleotides to the basic htel-21 DNA sequence of $\mathrm{G}_{3}\left(\mathrm{TTAG}_{3}\right)_{3}$ induces diverse folds [8]. For instance, TA(htel-21) was found by NMR technique to mostly fold into the hybrid-1 form (Figure 2,3), whereas the TA(htel21)TT adopted the hybrid-2 topology (Figure 2, 4) as the dominant, but not the only form. The hybrid structures are generally polymorphic in solution and are interconvertible due to the small energetic differences between them [84]. Incorporation of $\mathrm{br}^{8} \mathrm{dG}$ into selected syn $\mathrm{dG}$ position(s) converted the polymorphic fold into a stabilized majority fold. For example, the syn dG16 in the middle tetrad of the TA(htel-21) and the syn dG15 in one of the terminal tetrads of the TA(htel-21)TT GQs greatly stabilized their respective major topologies, according to Phan et al. [71]. Another noteworthy illustration for the effect of $\mathrm{br}^{8} \mathrm{dG}$ was the induction of conformational changes in GQs. Using the same 25-mer TA(htel-21)TT, An et al. [87] incorporated two $\mathrm{br}^{8} \mathrm{dGs}$ into the sequence. The dual incorporation induced exclusively either the hybrid-1 or the hybrid- 2 fold in $\mathrm{K}^{+}$(wt for the wild-type):

\section{Wild-type (wt): TA GGG TTAGGG TTAGGG TTAGGG TT \\ $\mathrm{br}^{8} \mathrm{dG}$ in G16, G21: TA GGG TTAGGG TTAGbr ${ }^{8} \mathrm{GG}$ TTA $\underline{b^{8}}{ }^{8}$ GG TT hybrid-1}

$\mathrm{br}^{8} \mathrm{dG}$ in G10, G15: TA GGG TTAGbr ${ }^{8} \mathrm{GG}$

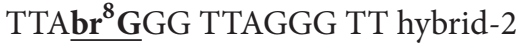

$\mathrm{br}^{8} \mathrm{dG}$ impacted both the topology and stability when incorporated into tetramolecular parallel GQs (Figure 2, 6). $\mathrm{TG}_{3} \mathrm{~T}$ molecules in $\mathrm{K}^{+}$fold into [TGGGT] ${ }_{4}$. Esposito et al. [67] separately substituted each $\mathrm{G}$ of $\mathrm{TG}_{3} \mathrm{~T}$ with $\mathrm{br}^{8} \mathrm{G}$. All three modified sequences assembled into tetramolecular GQs; the stabilities and topologies were, however, different. CD spectrum of [ $\left.\mathrm{Tbr}^{8} \mathrm{GGGT}\right]_{4}$ showed, surprisingly, antiparallel characteristics and its thermal stability was much higher, by $13.3^{\circ} \mathrm{C}$, than that of the native parallel tetramolecular scaffold. The $\left[\mathrm{TGbr}^{8} \mathrm{GGT}\right]_{4}$ formed parallel GQ architecture, as the native sequence did, and stabilized the native structure by $3.6^{\circ} \mathrm{C}$. The TGGbri ${ }^{8}$ GT proved unstructured, and no $T_{m}$ could be determined. It was also an unusual finding that the $\operatorname{br}^{8} \mathrm{G}$ tetrads were all syn with each tetramolecular analog, while the other two G-tetrads of the GQ were anti, as in the case of the unmodified $[\text { TGGGT] }]_{4}$.

8-Methyl-2'-deoxyguanosine $\left(m^{8} d G\right)$. Another stabilizing guanine analog is the 8-methylguanine nucleoside $\left(\mathrm{m}^{8} \mathrm{dG}\right.$ ) (Figure 6,3 ), and as with the $b^{8} d G$, it is stabilizing only if it is substituted for syn dGs. $\mathrm{m}^{8} \mathrm{G}$ has been, however, much less utilized than the $\mathrm{br}^{8} \mathrm{G}$. He et al. [33] incorporated a methyl (and a 1-propynyl) group into the C8 positions of G1, G5, G10, and G14 of the 15-mer TBA DNA oligonucleotide (Figure 2,7), where positions were all syn. Thermal stability of the unmodified TBA GQs, $55^{\circ} \mathrm{C}$, was increased to $70^{\circ} \mathrm{C}$ by these substitutions. Concomitantly, the substitutions increased the thrombin-binding activity as well, presumably by the stabilization of the chair-like structure. $\mathrm{Xu}$ and Sugiyama [95] incorporated the $\mathrm{m}^{8} \mathrm{dG}$ into the 18 -mer CGGGGGGTTTTGGGCGGC of the G-rich termini of retinoblastoma gene DNA. Thermal stability of the two-tetrad antiparallel GQ increased when syn dGs were replaced and decreased when an anti $\mathrm{dG}$ was substituted by $\mathrm{m}^{8} \mathrm{dG}$. The GQ stability pays energy penalty for accommodating a syn $\mathrm{dG}$ analog in place of an anti $\mathrm{dG}$. Later, $\mathrm{m}^{8} \mathrm{dG}$ was incorporated into the tetramolecular GQ-forming $\mathrm{TG}_{3} \mathrm{~T}$ and $\mathrm{TG}_{4} \mathrm{~T}$ oligodeoxynucleotides (Figure 2, 6). Using $\left[\right.$ TGGGT] $_{4}$, Virgilio et al. $[96,97]$ and Tran et al. [98] determined that $\mathrm{m}^{8} \mathrm{dG}$ had a position-dependent effect on folding arrangements. $\mathrm{N}$-glycosidic linkages of the $\mathrm{G}$ and $\mathrm{T}$ nucleosides are all anti in the native fourfold parallel GQs. In position 2, however, $\mathrm{m}^{8} \mathrm{dG}$ formed an all-syn tetrad that resulted in an antiparallel-type $\mathrm{CD}$ spectrum in $\mathrm{K}^{+}$, that is, a large positive band near $290 \mathrm{~nm}$ and a negative one near $260 \mathrm{~nm}$ (Figure 3). The $\mathrm{m}^{8} \mathrm{dG}$-tetrad in position 3 was all anti, the GQ was parallel, and the CD spectrum showed large positive peak at $260 \mathrm{~nm}$ and a small negative one around $240 \mathrm{~nm}$. $\mathrm{m}^{8} \mathrm{dG}$ in position 4 hindered the GQ formation. Results were similar to those observed with $\mathrm{br}^{8} \mathrm{dG}$. The authors also studied $\mathrm{m}^{8} \mathrm{dG}$ in position 2 of the anti-HIV aptamer TGGGAG (Hotoda aptamer) and its $3^{\prime}$ T-extended sequence TGGGAGT and observed thermal stabilization. Again, allsyn $\mathrm{m}^{8} \mathrm{dG}$ tetrads were observed in both GQs; however, the topologies remained parallel [99]. Structure and kinetics of formation of the tetramolecular GQ model were investigated using $\mathrm{m}^{8} \mathrm{G}$ in another study. $\mathrm{m}^{8} \mathrm{dG}$ at the $5^{\prime}$-end of the short oligonucleotide accelerated GQ formation by 15 -fold relative to the unmodified oligonucleotide [98]. In a recent study, Zhao et al. [100] substituted dGs by $\mathrm{m}^{8} \mathrm{dGs}$ in the GQs 
formed by an htel, the TBA, and the c-myc promoter's Grich pu27 sequences. $\mathrm{m}^{8} \mathrm{dG}$ proved significantly stabilizing in those cases when it replaced syn dGs and the stabilization was cumulative when two or three $\mathrm{m}^{8} \mathrm{dG}$ s were substituted for syn $\mathrm{dGs}$. However, $\mathrm{m}^{8} \mathrm{dG}$ proved destabilizing when an anti $\mathrm{dG}$ was replaced.

8-O-Methyl-2'-deoxyguanosine ( $\left.\mathrm{om}^{8} \mathrm{dG}\right)$. Lech and coworkers [80] studied the influence of 8-O-methylguanine deoxyguanosine (Figure 6,4) on GQ structures using the 24mer model TT(htel-21)A that forms a predominant hybrid-1 topology (Figure 2, 3) in $\mathrm{K}^{+}$solution [101]. Inserted one by one into six different positions (bold) into the 24-mer

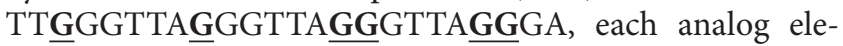
vated the thermal stability of the GQ by an average of $3.1 \pm$ $1.4^{\circ} \mathrm{C}$, with slight positional effects. Except for position 22, each site was syn. The guanine analog in the anti dG22 position also elevated the stability, although somewhat less than in the 21 syn position. The hybrid-1 topology was not basically changed by $\mathrm{om}^{8} \mathrm{dG}$.

8-Amino- $2^{\prime}$-deoxyguanosine $\left(n^{8} d G\right)$. The effect of the synpreferring $\mathrm{n}^{8} \mathrm{dG}$ on GQ stability (Figure 6, 5) was first described using the TBA GQ by De La Osa et al. [102]. The analog was placed (for MD calculation) and incorporated into position 2, which is an anti dG position of the GQ. Molecular dynamics and thermodynamic integration calculations $(\mathrm{MD} / \mathrm{TI})$ suggested that the $\mathrm{n}^{8} \mathrm{dG}$ substitution led to destabilization by $1.2-1.9 \mathrm{kcal} / \mathrm{mol}$ in the $\Delta \Delta G^{\circ}$ value, and the nature of the central cation $\left(\mathrm{Na}^{+}\right.$or $\left.\mathrm{K}^{+}\right)$did not have substantial effect. CD-based melting profiles confirmed the MD/TI calculations. The unmodified GQ had a $T_{m}$ value of $46^{\circ} \mathrm{C}$, while the $\mathrm{n}^{8} \mathrm{dG}$-modified one had $39^{\circ} \mathrm{C}$. The loss in folding free energy by the single substitution corresponded to $1.2 \mathrm{kcal} / \mathrm{mol}$, in perfect agreement with the MD/TI calculations. This destabilization was enthalpic in nature, with a $\Delta \Delta H^{\circ}$ of $5.5 \mathrm{kcal} / \mathrm{mol} . \mathrm{n}^{8} \mathrm{dG}$ was later incorporated into the tetramolecular parallel GQ models built from $\mathrm{TG}_{4} \mathrm{~T}$ and $\mathrm{TG}_{5} \mathrm{~T}$ and an unusual position-dependent effect was observed by Gros et al. [73]. In the $\left[\mathrm{TG}_{n} \mathrm{~T}\right]_{4}$, the $\mathrm{n}^{8} \mathrm{dG}$ tetrads reduced the thermostability in three of the four $G$ positions and elevated the stability only in position 3 of the $\mathrm{TG}_{4} \mathrm{~T}$ sequence. With another, on the $5^{\prime}$-end covalently linked tetramolecular assembly, Ferreira and coworkers [103] found that inserting a single $n^{8} \mathrm{G}$ only into one strand in position 2 or 3 stabilized the parallel GQ. In both cases, $n^{8}$ G-G-G-G tetrads were formed:

\section{$3^{\prime}$-T묘 ${ }^{8}$ GGGGTT- $5^{\prime}$-TB- $\left[5^{\prime} \text {-TTGGGGT- } 3^{\prime}\right]_{3}$, $3^{\prime}$-TG ${ }^{8}$ GGGTT- $5^{\prime}$-TB- $\left[5^{\prime} \text {-TTGGGGT- } 3^{\prime}\right]_{3}$,}

where TB was the Trebler linker [O-phosphate- $\mathrm{CH}_{2}$ $\left.\mathrm{C}\left(\mathrm{CH}_{2} \mathrm{OCH}_{2} \mathrm{CH}_{2} \mathrm{CH}_{2} \mathrm{O} \text {-phosphate }\right)_{3}\right]$. With the intramolecular, mostly hybrid-1 GQ of TT(htel-21)A, Lech et al. [80] found that $\mathrm{n}^{8} \mathrm{dG}$ was only marginally stabilizing when incorporated into the syn $\mathrm{dG}$ positions of $3,9,15,16$, and 21 with the average $\Delta T_{m}$ of $1.1 \pm 1.1^{\circ} \mathrm{C}$. According to the CD spectra the hybrid-1 type, topology (Figure 2, 3) apparently was not changed by any of these substitutions.
8-Propynyl Derivatives of $2^{\prime}$-Deoxyguanosine. The triple C-C bond-containing propynyl group in conjugation with heteroaromatic bases, such as the uracil in duplexes [104] as well as in the TBA GQs [52], greatly enhanced the stability of the macromolecular structure through strengthening the stacking. The propynyl group in conjugation with guanine (Figure 6, 6) also stabilized the GQ, which was again the TBA GQ, when incorporated into all four syn dG positions, G1, G5, G10, and G14, of the 15-mer sequence. The substitutions increased the thrombin-binding activity of modified TBA GQ, as compared to the wild type, probably due to the stabilization of the intramolecular GQ structure. Larger substituent groups in these positions, like phenyl-ethynyl, however, decreased the activity, probably due to steric hindrance [33].

8-Vinyl-2'-deoxyguanosine $\left(v^{8} d G\right)$ and Its Derivatives. The fluorescent base 8 -vinylguanine $\left(\mathrm{v}^{8} \mathrm{G}\right)$ (Figure 7,1$)$ proved to be a good alternative to the widely used fluorescent marker 2aminopurine $\left(\mathrm{n}^{2} \mathrm{Pu}\right.$, Figure 8,8$)[105,106]$. Nadler et al. [105] incorporated the $\mathrm{v}^{8} \mathrm{dG}$ into positions G3, G15 (middle tetrad), and G4 (terminal tetrad) of the htel-23 A(htel-21)T. In $135 \mathrm{mM} \mathrm{Na}^{+}$solution, the two middle tetrad substitutions elevated the thermal stability of the wild type $\left(T_{m}=56^{\circ} \mathrm{C}\right)$ by $3-4^{\circ} \mathrm{C}$ and $v^{8} \mathrm{G}$ in one of the two terminal tetrads by $2^{\circ} \mathrm{C}$. In $100 \mathrm{mM} \mathrm{K}^{+}$only the vinyl analog of the middle tetrad (position 15) increased the stability by $5^{\circ} \mathrm{C}$ of the unmodified GQ $\left(T_{m}: 61^{\circ} \mathrm{C}\right)$. CD spectra of the three modified GQs in $\mathrm{Na}^{+}$looked hybrid types contrary to the wild-type's antiparallel spectrum (Figure 3 ). The wild-type htel-23 folds into hybrid conformations in $\mathrm{K}^{+}$solution. The middle tetradmodified GQ studied followed the trend in $\mathrm{K}^{+}$, whereas the CD spectrum of the terminal tetrad-modified GQ was antiparallel type [105]. 8-Vinylguanine was also attached to 2-aminoethylglycine, creating a peptide nucleic acid (PNA) building block. This allowed the differentiation between topologies of GQs based on fluorescence intensity. $\mathrm{v}^{8} \mathrm{dG}$ was found to be capable of adopting both syn and anti conformations required by distinct GQ structures [106].

Aromatic derivatives of 8-vinylguanine, the 8-(2-phenylethenyl)- and 8-[2-(pyrid-4-yl)-ethenyl]guanines (Figure 7, 2, 3), have been studied by Dumas and Luedtke [107] by incorporating them into positions 9 and 23 of the 24-mer TT(htel-21)A. Although $\mathrm{v}^{8} \mathrm{dG}$ can adopt both syn and anti conformations $[105,106]$, the two aromatic derivatives of $\mathrm{v}^{8} \mathrm{dG}$ adopted only anti glycosidic conformation in solutions, like $\mathrm{dG}$ does, contrary to other 8-modified dGs, such as $\mathrm{br}^{8}-, \mathrm{m}^{8}-, \mathrm{n}^{8}-$, or $\mathrm{o}^{8} \mathrm{dG}$. Due to the conjugated vinyl tethers, the bulky aryl and heteroaryl groups are detached far enough from $\mathrm{dG}$ not to hinder the energetically preferred anti orientation of dG. The GQ of TT(htel-21)A has been described to fold into hybrid-1 topology (Figure 2, 3) in $\mathrm{K}^{+}$ and into the antiparallel topology in $\mathrm{Na}^{+}$(Figure 2, 1) [107]. The position 9-substituted 24-mer GQ was stabilized by the two ethenyl derivatives by $7-8^{\circ} \mathrm{C}$ in $\mathrm{Na}^{+}$and $5-8^{\circ} \mathrm{C}$ in $\mathrm{K}^{+}$; however, in position 23 , the GQs were destabilized by $1-6^{\circ} \mathrm{C}$ in $\mathrm{Na}^{+}$and $6-8^{\circ} \mathrm{C}$ in $\mathrm{K}^{+}$. In position 9 , the two G-analogs did not change the $\mathrm{CD}$ spectrum of the wild-type GQs 


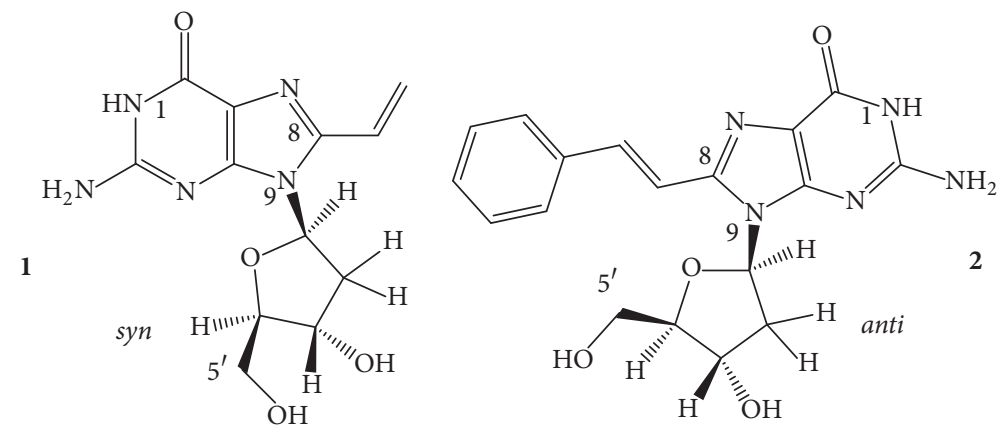

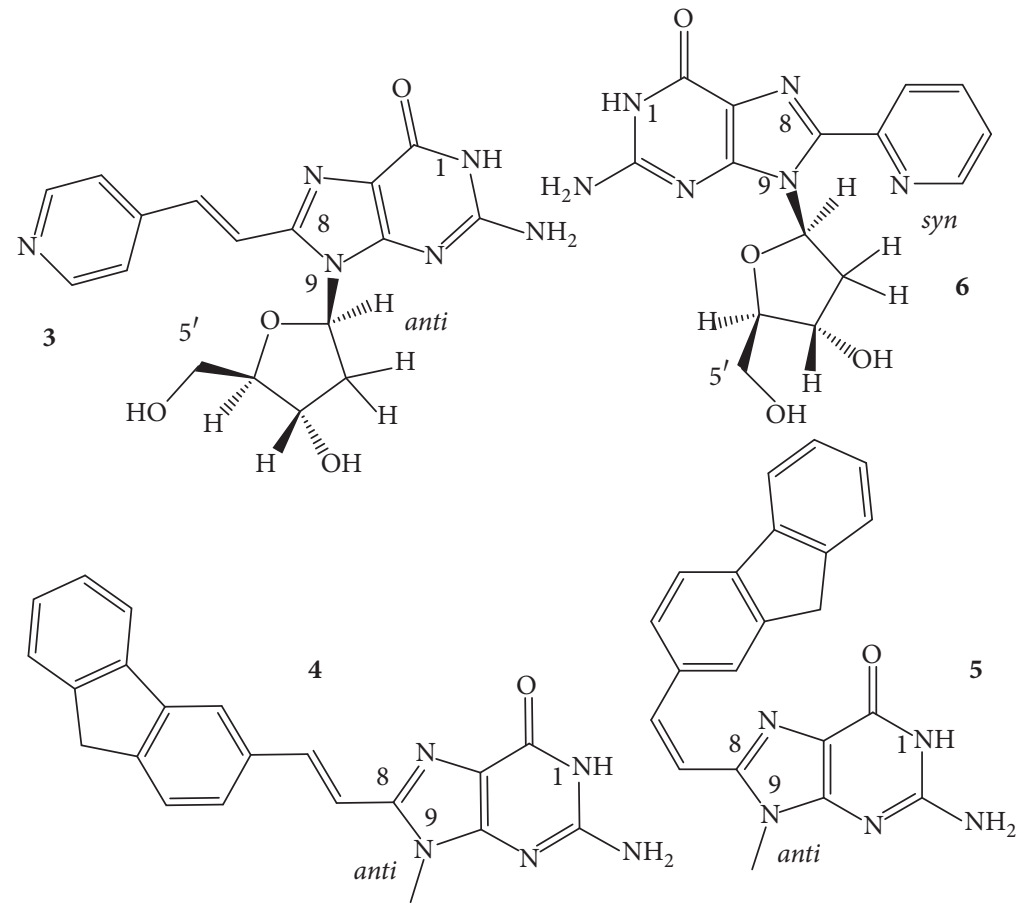

FIGURE 7: Structure of the syn 8-vinyl-2-deoxyguanosine (1); the anti 8-(2-phenylethenyl)- (2) and 8-[2-(pyrid-4-yl)-ethenyl]-dGs (3); the cis (4) and trans isomers (5) of the anti 8-vinyl-substituted-dGs; and the syn 8-(2-pyridyl)-dG (6).

(hybrid), whereas the position 23-substituted GQs showed antiparallel-type spectra in $\mathrm{K}^{+}$. In $\mathrm{Na}^{+}$, the modified GQs remained antiparallel like the wild-type htel-24 GQ did [107]. 8-Fluoroenylvinylguanine $\left(\mathrm{Fv}^{8} \mathrm{G}\right)$, another derivative of $\mathrm{v}^{8} \mathrm{G}$, was also incorporated into GQ-forming oligonucleotides. Ogasawara and Maeda [108] developed a light-controlled reversible folding-unfolding GQ structure with this analog based on the cis-trans isomerization of a photochromic nucleobase $\mathrm{Fv}^{8} \mathrm{G}$ (Figure 7, 4,5), incorporated into the 15-mer TBA sequences, the GGTTGFv ${ }^{8}$ GTGTGFv $^{8} \mathbf{G}$ TTGG and $\mathrm{GFv}^{8} \mathbf{G}$ TTGGTGTGGTTGFv $\mathbf{v}^{8} \mathbf{G}$.

The native TBA sequence folds into a chair-type, twotetrad antiparallel GQ in $\mathrm{K}^{+}$(Figure 2), and its $\mathrm{CD}$ spectrum was only slightly changed by the trans-Fv ${ }^{8} \mathrm{G}$ (Figure 7,4 ) in anti $\mathrm{dG}$ positions, G6 and G11 and G2 and G15. $T_{m}$ of the modified GQs increased by $\sim 10^{\circ} \mathrm{C}$, up to $\sim 60^{\circ} \mathrm{C}$. On irradiation at $410 \mathrm{~nm}$, the trans- $\mathrm{Fv}^{8} \mathrm{G}$ changed into the cis form (Figure 7,5), which resulted in unfolding of the stable GQs. Irradiation at $310 \mathrm{~nm}$ reversed the unfolding. 8-(2Pyridyl)guanine $\left(\mathrm{Py}^{8} \mathrm{G}\right.$, Figure 7,6$)$ is a highly fluorescent compound that has been used to study the folding of the 24mer $\mathrm{TTG}_{3}\left(\mathrm{TTAG}_{3}\right)_{3} \mathrm{~A}$ GQs [109]. Positions G9 and G23 of terminal tetrads were syn in $\mathrm{Na}^{+}$; still the syn $\mathrm{Py}^{8} \mathrm{dG}$ affected the thermostability in altered ways: $9-\mathrm{Py}^{8} \mathrm{dG}$ increased $T_{m}$ of the wild-type GQ by 7 and $10^{\circ} \mathrm{C}$ in $\mathrm{Na}^{+}$and $\mathrm{K}^{+}$, respectively, whereas $23-\mathrm{Py}^{8} \mathrm{dG}$ reduced the wild-type's $T_{m}$ by 2 and $9^{\circ} \mathrm{C}$, respectively. Apparently, the glycosidic orientation of the $\mathrm{Py}^{8} \mathrm{dG}$ was not related to these effects. $9-\mathrm{Py}^{8} \mathrm{G}$ in $\mathrm{K}^{+}$did not change the wild-type's hybrid fold; however, $23-\mathrm{Py}^{8} \mathrm{G}$ induced a conversion into antiparallel topology in $\mathrm{K}^{+}$.

The "push-pull" emissive fluorophores, which can exhibit environmentally sensitive quantum yields due to excitedstate proton-transfer reactions with bulk solvent, were thoroughly investigated by Manderville's and Wetmore's groups. 8-Furyl- and 8-(4-cyanophenyl)-2' -deoxyguanosines (Figure $8,1,2)$ were used to monitor the duplex-GQ exchanges. In a syn $\mathrm{dG}$ position of the TBA GQ (pos. 5), the syn 8-aryl dGs significantly stabilized the GQ in both $\mathrm{Na}^{+}$and $\mathrm{K}^{+}$ solutions, whereas they substantially destabilized it when incorporated into an anti dG location (pos. 6) [110, 111]. The 


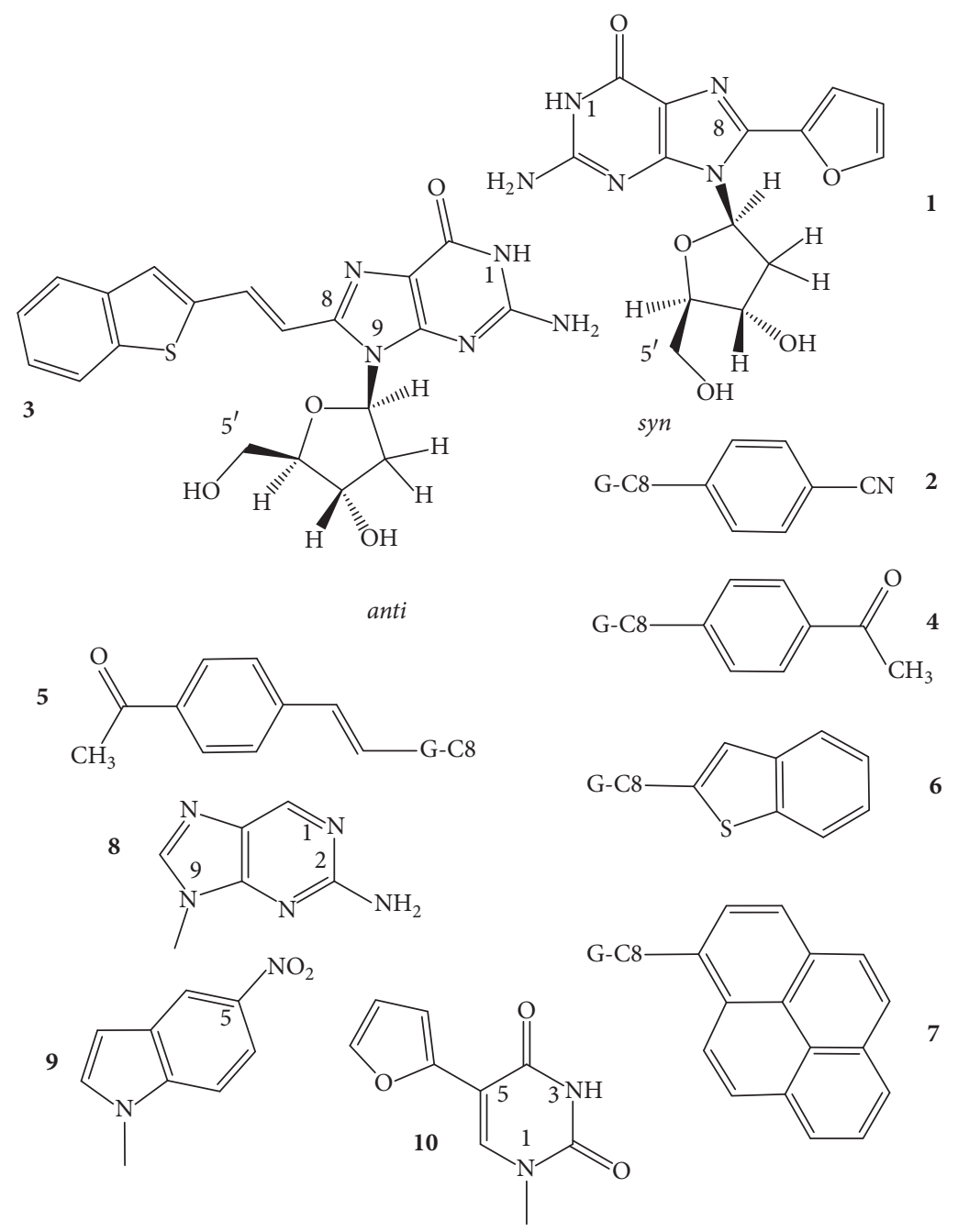

FiguRE 8: Structures of syn 8-furyl-dG (1); 8-(4-cyanophenyl)guanine (2); anti 8-vinyl-benzothienyl-dG (3); 8-acetylphenylguanine (4); 8vinyl-acetylphenylguanine (5); 8-benzothienylguanine (6); 8-(pyren-1-yl)guanine (7); 2-aminopurine (8); 5-nitroindole (9); and 5-furyl-2' deoxyuridine (10).

8-furyl- and 8-vinyl-benzo(b)thienyl-dGs (Figure 8, 1, 3), which, based on analogy, are assumed to be syn and anti dGs, respectively, were incorporated into both syn and anti $\mathrm{dG}$ positions of the TBA DNA. 8-Furyl-dG in the syn positions of 10 and 14 elevated $T_{m}$ of TBA GQ, while the more lipophilic benzothienyl derivative decreased it slightly in the syn G5 position and more extensively in the anti G6 position. Double labeling with furyl and benzothienyl derivatives enhanced the destabilization when one of the analogs was in anti position; however, when both probes substituted for syn dG positions, the wild type was significantly stabilized [112]. The pushpull phenomenon in the TBA GQ was also studied with acetylphenyl, benzo[b]thienyl, quinolyl, pyren-1-yl (Figure 8, 7 ), and the 8-vinyl tethered derivatives of acetylphenyl and benzo[b]thienyl attached to C8 of dG (Figure 8). The dG analogs were substituted for syn dGs of the TBA DNA sequence. The aromatic and heteroaromatic groups with the aryl ring attached directly to C8 of guanine thermodynamically favor the syn glycosidic conformations and when these analogs substituted for the syn dG5 they increased the thermostability of the wild-type TBA GQ. The two aromatic analogs tethered to guanine base via the conjugated vinyl group favored anti glycosidic torsion angle (Figure 8, 3, 5). The anti 8-vinyl-acetylphenyl-dG slightly decreased $T_{m}$ of the wild type in the syn dG5 position but moderately elevated it in the anti dG6 position [113]. Similarly, the fluorescent 8-pyrrolyl-, 8-furyl-, 8-thienyl-, 8-benzofuryl-, 8indolyl-, and 8-benzothienyl-dGs were also incorporated into the TBA sequence in another study. These nucleosides also preferentially adopt syn conformation in solution and their insertion into the syn dG5 enhanced the thermal stability of the parent GQ in $\mathrm{K}^{+}$solution by $1-11^{\circ} \mathrm{C}$ and did not perturb the thrombin-binding affinity. 8-Thienyl-dG was found to increase the thermostability in each of the four syn positions by $7-10^{\circ} \mathrm{C}$ and its double incorporation by $18.5^{\circ} \mathrm{C}$, and substituting all four syn $\mathrm{dGs}, \Delta T_{m}$ was higher than $36^{\circ} \mathrm{C}$ [114]. Stability changes resulting from the syn versus anti glycosidic conformations of $\mathrm{dG}$ derivatives were similar 
to 8-(p-cyanophenyl)-dG in TBA and 8-furyl-dG in A(htel21) GQ systems [115]. 8-Furylguanine was also incorporated into each of the three tetrads of A(htel-21) and the effects of salts and cosolvents (acetonitrile, PEG-600, and N-methylmesoporphyrin) were examined on the structure. In addition to the influence of the syn and anti positions on stability, the position of the tetrad substituted affected the stability too [116]. The effect of 8-vinyl-acetylphenyl- and 8-vinylbenzothienyl-dGs on the TBA GQ system was also investigated for the detection of divalent metal ions. Based on its emission sensitivity to $\mathrm{Pb}^{2+}$, the vinyl-benzothienyl derivative proved to be an effective and selective sensor for $\mathrm{Pb}^{2+}$, having higher affinity for $\mathrm{Pb}^{2+}$ than for $\mathrm{Na}^{+}, \mathrm{K}^{+}$, or other bivalent cations of biological importance [117].

The C8-aryl- and heteroaryl substituents, such as C8phenyl, pyridine, thiophene, and furan of $\mathrm{dG}$, were also studied by Dumas and Luedtke [118], who used the changes in fluorescence to characterize the metal-binding affinity and specificity of the 8-substituted guanines in duplex and GQ DNAs as well as to study the effect on stability. Thermal stability of the GQ formed by the cKit sequence $\mathrm{AG}_{3} \mathrm{AG}_{3} \mathrm{C}_{\mathbf{X}} \mathrm{CTG}_{2} \underline{\mathbf{X}} \mathrm{AG}_{2} \mathrm{AG}_{3}$ substituted in positions 10 and $15(\underline{X})$ was investigated. 8-(2-Pyridinyl)guanine (Figure 7, 6) decreased $T_{m}$ of unmodified GQ $\left(64^{\circ} \mathrm{C}\right)$ by $2-4^{\circ} \mathrm{C}$ in positions 10 and 15 . This analog has also been used to study the folding properties of the 24-mer TT(htel-21)A GQ [109]. Position G23 in the $3^{\prime}$-terminal tetrad was syn in $\mathrm{Na}^{+}$; still the syn-

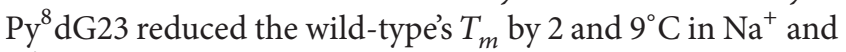
$\mathrm{K}^{+}$, respectively. Apparently, the glycosidic orientation of the $\mathrm{Py}^{8} \mathrm{dG}$ was not related to the effect. The $\mathrm{Py}^{8} \mathrm{G} 23$ induced a conversion of the hybrid fold(s) into antiparallel topology in $\mathrm{K}^{+}$.

2-Aminopurine $\left(n^{2} P u\right)$. The fluorescent 2 -aminopurine $\left(\mathrm{n}^{2} \mathrm{Pu}\right.$, a $\mathrm{G}$ or $\mathrm{A}$ analog) (Figure 8, 8) incorporated into the loop sequence of a potentially GQ-forming oligodeoxynucleotide has significantly enhanced fluorescent emission upon the formation of the GQ. (Its fluorescence is quenched in duplex DNA due to the stacking with flanking bases and becomes comparable to that of the free $n^{2} \mathrm{Pu}$ upon the formation of GQ as the $\pi$-stacking within the loops is distorted and effective quenching cannot occur.) $\mathrm{n}^{2} \mathrm{Pu}$ has been widely used in structural analysis of GQs. For example, for conformational studies, $\mathrm{n}^{2} \mathrm{Pu}$ was substituted separately for all four adenines in $\mathrm{A}$ (htel-21) [84, 119-121]. The analog did not change the folding and only slightly reduced the thermal stability of the parent GQ [122], while significant changes were observed in fluorescence intensity of $\mathrm{n}^{2} \mathrm{Pu}$ depending on whether it was present in the GQ or in the duplex formed with the complementary strand. $\mathrm{n}^{2} \mathrm{Pu}$ could also distinguish between the basket-type and propeller-type GQs. 2-Aminopurine has also been used to develop a sensitive fluorescent GQ assay for uracil DNA-glycosylase activity [123]. 2-Aminopurine was also substituted for Ts of the loops the 15-mer TBA and the $\left(G_{3} T\right)_{3} G_{3}$ oligonucleotides to develop fluorescent detection methods. The two oligomers folded into antiparallel and parallel GQs, respectively, in $\mathrm{K}^{+}$. The substitutions did not change the original topologies but changed the stabilities of the wild-type Qs. $T_{m}$ of $48^{\circ} \mathrm{C}$ for wild-type TBA GQ in $50 \mathrm{mM}$ $\mathrm{KCl}$ was increased by $2^{\circ} \mathrm{C}$ when substituting for $\mathrm{T} 3$ and $\mathrm{T} 2$ of the loops, in which positions the $\mathrm{T}$ is stacked to the core [1820]. In the other $\mathrm{T}$ positions, the $\mathrm{n}^{2} \mathrm{Pu}$ reduced the stability to around $40^{\circ} \mathrm{C} . T_{m}$ of the $\left(\mathrm{G}_{3} \mathrm{~T}\right)_{3} \mathrm{G}_{3} \mathrm{GQ}$ measured in $10 \mathrm{mM}$ $\mathrm{KCl}$ was $90^{\circ} \mathrm{C}$ and was reduced in all three loop $\mathrm{T}$ positions by $\mathrm{n}^{2} \mathrm{Pu}$. Single substitutions caused $4-5^{\circ} \mathrm{C}$ decrease in $T_{m}$, while double and triple substitutions caused a decrease of $12^{\circ} \mathrm{C}$ and $16^{\circ} \mathrm{C}$, respectively [124]. Similarly, $\mathrm{n}^{2} \mathrm{Pu}$ substituting for G8 of the central TGT loop just slightly reduced the $T_{m}$ value of $52.4^{\circ} \mathrm{C}$ of the native GQ to $51.6^{\circ} \mathrm{C}$, but the modified TBA GQ proved more stable based on the free energy change $\left(\Delta G_{20}^{\circ}\right)$ value from -2.3 to $-3.6 \mathrm{kcal} / \mathrm{mol}$ [125].

5-Nitroindole. Tsvetkov et al. [126] incorporated the universal base 5-nitroindole (Figure 8,9 ) into various positions of the TBA oligonucleotide. All modified TBAs formed antiparallel GQ structures and retained the ability to inhibit thrombin. UV absorption-based thermal stability of T7 substitution resulted in $T_{m}$ of $43.0^{\circ} \mathrm{C}$ and of the $\mathrm{T} 9$ in $38.5^{\circ} \mathrm{C}$, while $T_{m}$ of the native TBA GQ was $51.9^{\circ} \mathrm{C}$. On the other hand, replacement by 5 -nitroindole of the anti G8 retained the stability of the native TBA GQ and this substitution substantially increased the clotting time and resulted in a twofold lower $\mathrm{IC}_{50}$ value, as compared to the unmodified TBA GQ. Attachment of 5-nitroindole either to the $5^{\prime}$ or to the $3^{\prime}$-terminus did not change the stability of the unmodified GQ, 51.1 and $51.2^{\circ} \mathrm{C}$, respectively [126].

3.1.2. Pyrimidine Derivatives. Loop length and composition are the major factors that determine the stability and folding features of natural GQs $[61,127,128]$. For instance, a single one-nucleotide loop generally keeps the GQ in parallel topology $[129,130]$. With increasing loop lengths, thermodynamic stability generally decreases [128]. Effect of loop substitutions on GQ structures has been widely studied, but the majority of the investigations focused on the mutation by natural nucleotides, T/U, C, A, and I, to increase the loop length and change the sequence. The TBA DNA was among the most mutated GQs due to its stable and straightforward conformation [10]. In addition to 2-aminopurine, examined in the preceding section, those few loop base modifications are discussed here that have been carried out using the synthetic nucleotide analogs only, which increase or decrease the stability depending on the position of substitution. The natural base lesions that also form in the loops of GQs, such as $\mathrm{o}^{8} \mathrm{~A}, \mathrm{hm}^{5} \mathrm{U}$, and AP sites, are reviewed in Section 5.

The TBA DNA's six loop thymidines were replaced by a fluorescent furyl derivative, the 5 -furyl-2 ${ }^{\prime}$-deoxyuridine (Figure 8, 10), to demonstrate the analog's ability to determine the positional impact of each thymine on the stability and thrombin-binding activity of the TBA GQ [131]. Earlier NMR studies suggested that $\mathrm{T} 4$ and $\mathrm{T} 13$ stack strongly with the neighboring G-tetrad and T9 of GGTTGGTGTGGTTGG interacts with G8 of the TGT loop and with the G-tetrad. The T3, T7, and T12 do not interact with adjacent nucleotides and point outwards, towards the solvent [18-20]. Thermal stability of the modified TBA GQs showed good correlation with the steric predictions of the NMR studies. In $\mathrm{K}^{+}$solution, 


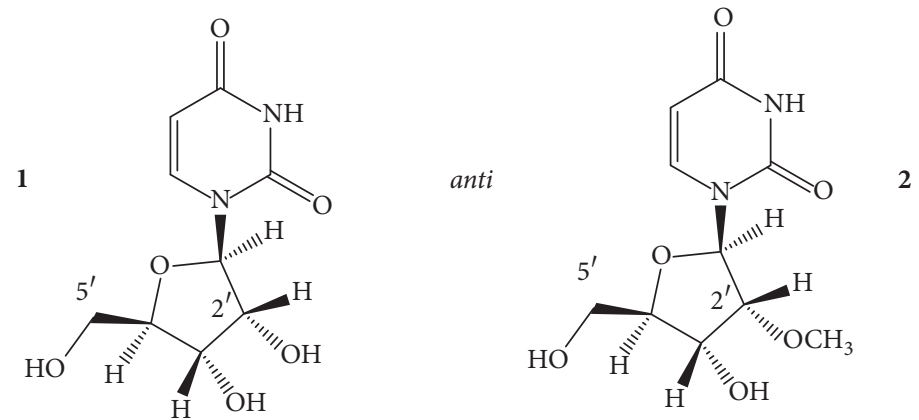

3
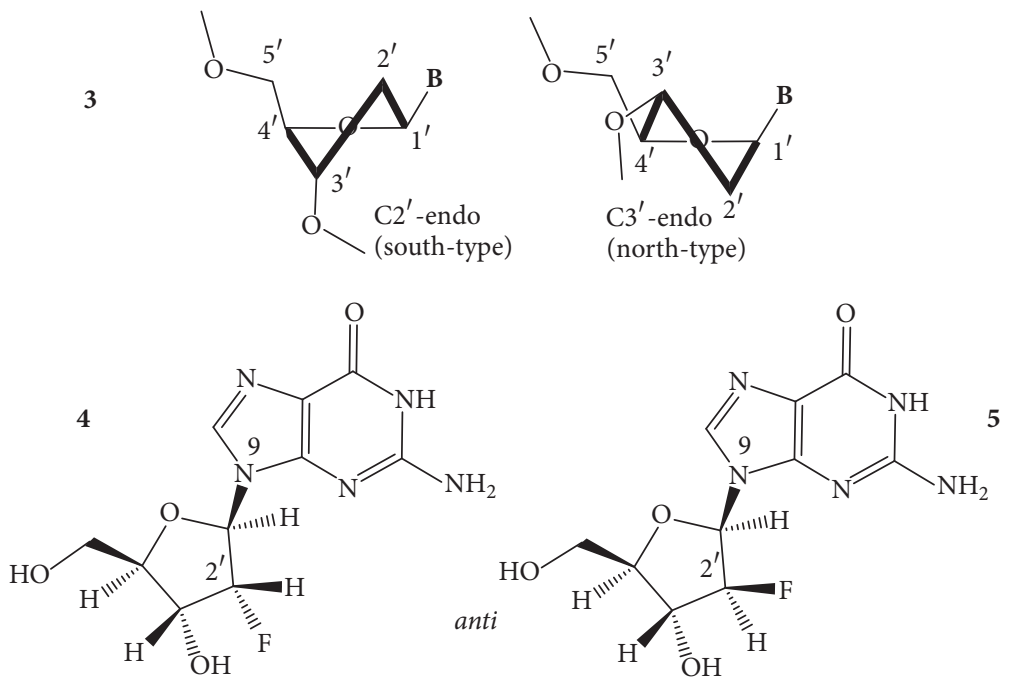

Figure 9: Structures of the anti ribo- (1) and the $2^{\prime}$-O-methylribouridines (2); the $\mathrm{C} 2^{\prime}$-endo (S-type) and $\mathrm{C} 3{ }^{\prime}$-endo (N-type) sugar puckers (3); the $2^{\prime}$-fluoro- $2^{\prime}$-deoxyribofuranosylguanine (4); and $2^{\prime}$-fluoro- $2^{\prime}$-deoxy-arabinofuranosylguanine (5).

the furyl analog in positions 4,9 , and 13 enhanced $T_{m}$ of the wild type by $6.5,3$, and $3^{\circ} \mathrm{C}$, respectively, while that in positions 3,7 , and 12 decreased it by -1.5 to $-2^{\circ} \mathrm{C}$. The slight reduction in $T_{m}$ probably originated from the stronger lipophilic character of the furyl derivative, relative to T's methyl group [130]. Structures of the intramolecular A(htel21 ) and the bimolecular $\left[\mathrm{G}_{4} \mathrm{~T}_{4} \mathrm{G}_{4}\right]_{2}$ GQs were investigated by using 5-iodouracil (io $\left.{ }^{5} \mathrm{U}\right)$ [132].

\subsection{Derivatives of the $\beta$-D-Furanose Sugar Moiety}

Ribonucleosides and 2'-O-Methyl-ribonucleosides. Tetramolecular GQs assembling from the ribo hexamer, abbreviated as $u_{4} \mathrm{u}$, were among the first ribo-GQs published. The GQ proved extremely stable in $50 \mathrm{mM} \mathrm{KCl} \mathrm{[133].} \mathrm{Later,} \mathrm{5-}$ bromouridine-containing tetramolecular structures, $\left[\underline{b r}^{5} \mathbf{u g}_{4} \mathrm{u}\right]_{4}$ $[134]$ and $\left[\mathbf{b r}^{5} \mathbf{u g a g g u}\right]_{4}$, were characterized by X-ray diffraction method. The latter GQ contained a g-u octad in addition to guanine and adenine tetrads [135]. The full ribo and the $2^{\prime}$ O-methylribo modifications (Figure 9, 1, 2) destabilized the monomolecular folds, like the GQs of the TBA, the htel-18 AGG(TTAGG) ${ }_{3}$, and the A(htel-21) GQs, and also bimolecular GQs in $\mathrm{Na}^{+}$, such as the $\left[\mathrm{dG}_{4} \mathrm{~T}_{4} \mathrm{G}_{4}\right]_{2}$, whereas these two modifications stabilized the tetramolecular parallel GQ formed by $\mathrm{TG}_{4} \mathrm{~T}$ oligonucleotides in both $\mathrm{Na}^{+}$and $\mathrm{K}^{+}$solutions [1]. Ribonucleosides, due to their thermodynamically preferred anti $\mathrm{N}$-glycosidic conformation, originating from the $\mathrm{C}^{\prime}$-endo sugar pucker (Figure 9, 3), can change the folding topology if incorporated into syn dG positions of deoxy-GQs, for instance, as described for the TBA GQ whose antiparallel fold could be changed to parallel and the unimolecular topology to bimolecular [136]. The full riboTBA GQ was parallel and was less stable than the deoxy.

Riboguanosines $(\mathrm{g})$ incorporated into syn positions of dG1 and dG9 and the anti dG4 and dG12 of the two terminal tetrads of the $G_{4} T_{4} G_{4}$ DNA GQ, which forms a bimolecular basket-type antiparallel GQ with four G-tetrads in both $\mathrm{Na}^{+}$ and $\mathrm{K}^{+}$[137], gave rise to $\mathrm{N}$-glycoside conformationdependent effect on stability in $\mathrm{K}^{+}$. Interestingly, substitution of syn dGs increased, while that of the anti dGs did not change $T_{m}$ of the wild-type GQ. A single $\mathbf{g}$ for a $\mathrm{G}$ changed the topology. With the exception of GGGGTTTTGGGg, which adopted an antiparallel structure, all hybrids formed parallel, bimolecular GQs $[138,139]$. For an NMR study, various GQforming sequences like the Myc and htel were systematically modified by single riboguanosine substitutions at anti dG positions. Folding topology of most native GQs changed on the formation of DNA-RNA hybrid structures. [140]. Zhou et al. [141] inserted riboguanosine tetrads into the parallel intramolecular $\mathrm{TTG}_{3} \mathrm{TG}_{3} \mathrm{TTG}_{3} \mathrm{TG}_{3} \mathrm{TT}$, the tetramolecular $\left[\mathrm{TG}_{3} \mathrm{~T}\right]_{4}$ and $\left[\mathrm{TG}_{4} \mathrm{~T}\right]_{4}$ GQs. Unexpected destabilizations 
were observed when the gggg quartets were at the $5^{\prime}$-end of the $\mathrm{G}$ stacks in both systems. However, gggg quartets replacing the other dG quartets stabilized the GQ structures.

A single 2 '-OMe modification significantly enhanced the thermal stability, by close to $10^{\circ} \mathrm{C}$, and also the peroxidase activity of the parallel GQ-forming sequence [B7]-3-0 [142]. Single uridine units were inserted into the three loops of the TBA GQ, separately. The uridine replacing thymidines in the central TGT loop resulted in an increased stability of the antiparallel GQ; however, substitution of thymidines in the TT loops induced destabilization of the TBA GQ [143].

$2^{\prime}$-Fluoro-2'-deoxyribofuranosyl- and $2^{\prime}$-Fluoro-2' ${ }^{\prime}$-deoxyarabinofuranosyl Nucleosides. Several laboratories have investigated the effect of $2^{\prime}$-deoxy-2' ${ }^{\prime}$-fluoro-D-arabinonucleotides on GQ structures $\left(\mathrm{fl}^{2 \prime}\right.$ araN), where $\mathrm{N}$ is any base (Figure 9, 5). The antiparallel TBA, the bimolecular $\left[\mathrm{G}_{4} \mathrm{~T}_{4} \mathrm{G}_{4}\right]_{2}$, and the tetramolecular phosphorothioate backbone-containing antiHIV $\left[\mathrm{T}_{2} \mathrm{G}_{4} \mathrm{~T}_{2}\right]_{4}$ were among the first GQ models examined [144]. The energetically preferred $\mathrm{N}$-glycosidic conformation of the $\mathrm{fl}^{2 \prime}$ araN is anti. Incorporation of $\mathrm{fl}^{2 \prime}$ araG or $\mathrm{fl}^{2 \prime}$ araT in place of anti dG-s of the tetrads or Ts of the loops in the antiparallel or parallel GQs stabilized the structure and maintained the fold of the wild-type GQ [144]. Lech's group [145] also investigated the effect of $\mathrm{fl}^{2 \prime}$ araG and the $2^{\prime}$-fluoro- $2^{\prime}$ deoxyguanosine, $\mathrm{fl}^{2 \prime} \mathrm{dG}$ (Figure 9, 4). The deoxy-GQ models were the hybrid-1 TA(htel-21) [101] and the hybrid-2 TA(htel21)TT in $\mathrm{K}^{+}$[146]. Substitution of the anti $\mathrm{dG}$ in the middle tetrad by either of the two nucleoside analogs increased the dominance of single folds and also enhanced the thermal stability by about $3^{\circ} \mathrm{C}$. However, when all five syn dGs were replaced by the analogs, the hybrid forms switched to parallel topology and the thermal stabilities increased by about $10^{\circ} \mathrm{C}$, as compared to the stability of the respective wild type. Single riboguanosine (g), arabinofuranosyl-guanine (araG), and $2^{\prime}$-deoxy-2'-fluoro-arabinofuranosylguanine $\left(\mathrm{fl}^{2 \prime}{ }^{\prime}\right.$ araG) replaced the dG9 of the 12-mer TAGGGTTAGGGT [147] that builds a mixture of two interconverting folds in $\mathrm{K}^{+}$ solution: a dimeric parallel and a dimeric antiparallel GQ [66]. The dG9 was syn in the antiparallel form; thus its replacement by the anti favoring sugar analogs converted the mixture to the dimeric parallel GQ with the concomitant increase in thermal stability from $41^{\circ} \mathrm{C}$ to $47-53^{\circ} \mathrm{C}$. The $\mathrm{fl}^{2 \prime}$ araG furnished the greatest stabilization, $12^{\circ} \mathrm{C}$ [145]. The effect of a single $2^{\prime}$-fluoro- $2^{\prime}$-deoxyguanosine $\left(\mathrm{fl}^{2 \prime} \mathrm{dG}\right), 2^{\prime}$ deoxy- $2^{\prime}$-fluoro-arabinofuranosylguanine $\left(\mathrm{fl}^{2 \prime}\right.$ araG), and $2^{\prime}$ $\mathrm{O}-4^{\prime}$-C-methylene-guanosine (LNA) (see next section and Figure 10,1$)$ was analyzed with more than 60 parallel and hybrid GQs [148]. Generally, substitutions of anti dGs of the G-tetrads increased the stability of GQs, while substitutions of syn positions disrupted the native GQ conformation. The 22-merGGGATGGGACACAGGGGACGGG oligonucleotide folds into a unimolecular hybrid-type GQ in $\mathrm{K}^{+}$ solution. $2^{\prime}$-Fluoro- $2^{\prime}$-deoxyguanosine $\left(\mathrm{fl}^{2 \prime} \mathrm{dG}\right)$, which also favors the anti glycosidic conformation, was substituted for the syn G1, G6, and G20 (bold, underlined) of the $5^{\prime}$-terminal tetrad. The substitutions changed the polarity of the tetrad. The overall fold, however, did not change and thus created a novel type of GQ in which all four G-columns comprised only syn or anti dGs: one column with all-syn and three Gcolumns with all anti glycosidic linkages [149]. Dickerhoff's group using $\mathrm{fl}^{21} \mathrm{dG}$ with the GQs of the previously applied 22-mer $\mathrm{G}_{3} \mathrm{ATG}_{3} \mathrm{ACACAG}_{3} \mathrm{GACG}_{3}$ and now also with the TT(htel-21)A confirmed the $\mathrm{fl}^{2 \prime} \mathrm{dG}$-induced conformational perturbations [150]. A single $\mathrm{fl}^{21} \mathrm{dU}$ was also inserted into the three loops of the TBA GQ. Substitution of thymidines in the TT loops by $\mathrm{fl}^{21} \mathrm{dU}$ resulted in destabilization of the TBA GQ [143].

Lietard et al. [151] synthesized the first microarrays containing $\mathrm{fl}^{2 \prime}$ araN and $\mathrm{fl}^{2 \prime}$ araN/DNA chimeric TBA oligonucleotides to fully map the binding affinity landscape of the TBA GQ. A series of promising $\mathrm{fl}^{2 \prime}$ ara-modified aptamer candidates were found with $K_{d}$ values that are significantly lower than that of the unmodified TBA GQ and which adopted highly stable, antiparallel GQ structures. The presence of $\mathrm{fl}^{21}$ araT at position T3 not only drastically strengthened the binding but also elevated the thermal stability of the unmodified TBA GQ by $7^{\circ} \mathrm{C}\left(T_{m}: 47^{\circ} \mathrm{C}\right)$. The TBA GQ could accommodate up to ten fluoro-ara nucleotides, in which TBA analogs were also promising aptamer candidates with dissociation constants up to three times lower than that of the wild-type TBA GQ. With these analogs' considerable increase, up to $20^{\circ} \mathrm{C}$ of GQ stability was observed and stable folding was observed even in the absence of $\mathrm{K}^{+}$ions. The T3 modification apparently preorganized the dinucleotide loop into the proper conformation for interaction with thrombin.

Locked Ribonucleosides (LNA). LNA is the abbreviation for locked nucleic acid. The LNA nucleoside has a modified ribose ring, which is locked in a stable $\mathrm{C} 3^{\prime}$-endo or $\mathrm{N}$-type (RNA-like) sugar pucker by a $2^{\prime}-\mathrm{O}-4^{\prime}-\mathrm{C}$-methylene linkage. Thus, the LNA contains a bicyclo-sugar moiety, resulting in a stable anti $\mathrm{N}$-glycosidic conformation of the nucleoside (Figure 10, 1). The LNA nucleosides increase the stability of duplex and triplex DNAs [152] but the B-type duplex is converted to the RNA-like A-type geometry [153]. LNA oligonucleotides have excellent hybridization properties with DNA and RNA oligomers. The structural impact of LNA in parallel GQs has been characterized by several groups [154, 155]. Nielsen et al. [156] used the telomeric sequence from the Oxytricha nova, $\mathrm{G}_{4} \mathrm{~T}_{4} \mathrm{G}_{4}$ for a $\mathrm{GQ}$ model. In $\mathrm{K}^{+}$this oligonucleotide forms a dimeric $\mathrm{GQ}$ with antiparallel G-columns and diagonal T4 loops $[157,158]$. The LNA-substituted sequence GLGLT ${ }_{4} G \underline{L} G \underline{L}$, where $\mathbf{L}$ stands for LNA-G, also formed a dimeric GQ, in which each G-stretch folded back into a Vshaped turn [159] and interacted with each of the three other G-stretches through formation of four G-tetrads. This new GQ folding topology was named the V4 fold. The V4 fold incorporates the features of both parallel and antiparallel GQs in one structure and the remarkable folding topology leads to tetrad steps with both coaligned (between outer and inner Gtetrads) and antialigned (between the two inner G-tetrads) hydrogen bonding, as in the parallel and antiparallel GQs, respectively. $\mathrm{CD}$ spectrum of the $\mathrm{V} 4$ fold thus displayed signatures of both parallel and antiparallel stacking [156]. In the intramolecular antiparallel, basket-type GQ-forming $\left(\mathrm{G}_{4} \mathrm{~T}_{4}\right)_{3} \mathrm{G}_{4}$, a single G-LNA or T-LNA reduced the stability of 


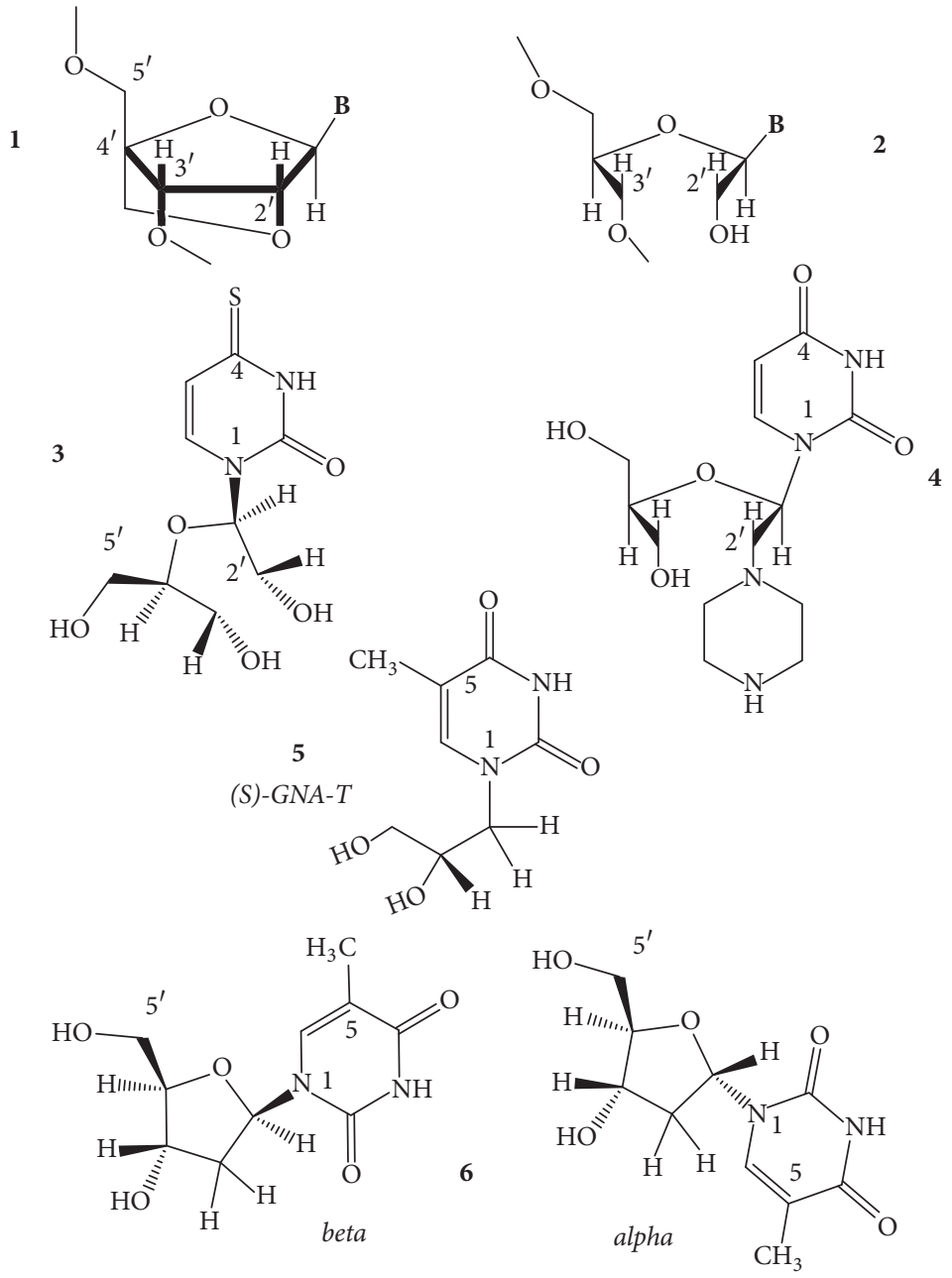

FIgURE 10: Structures of the locked nucleoside $2^{\prime}-\mathrm{O}-4^{\prime}-\mathrm{C}$-methylene-guanosine (LNA), where B stands for a nucleobase (1); the unlocked nucleoside $2^{\prime}$ - $3^{\prime}$-acyclic-ribonucleotide (UNA) (2); 4-thio-unlocked-uridine (3); $2^{\prime}$-C-piperazino-unlocked-uridine (4); an acyclic thymine derivative, (S)-GNA-T (5); and the beta- and alpha-thymidines (6).

the native GQ in a position-dependent way, most extensively by the G-LNAs [160]. A single G-LNA incorporated at the $3^{\prime}$ terminal G-position of TBA GQ did not change the topology but destabilized the wild-type GQ, by $6^{\circ} \mathrm{C}$, from $T_{m}$ of $52^{\circ} \mathrm{C}$ to $46^{\circ} \mathrm{C}$ [161]. The TBA GQ was also the model for G-LNA and T-LNA substitutions at G2 (anti), G5 (syn, both in the same G-tetrad), and T4 (in a TT loop), T7, and G8 (anti, in the TGT loop) positions. G-LNA at position G2 reduced $T_{m}$ of the unmodified GQ to the largest extent, from 48.1 to $33.5^{\circ} \mathrm{C}$ in $50 \mathrm{mM} \mathrm{KCl}$, although dG2 is anti. Substitution of T7 also reduced $T_{m}$ by about $5^{\circ} \mathrm{C}$, whereas G5-LNA (despite being a syn dG position) and G8-LNA (anti) increased the thermal stability by 2.6 and $1.9^{\circ} \mathrm{C}$, respectively. The T4-LNA GQ was unstable, and hence $T_{m}$ could not be determined. Thermal stability of the LNA-GQs was more or less inversely related to their antithrombin activity. The substitutions did not change the folding topology of the wild type as Bonifacio et al. reported [162]. The full LNA analog of the TBA DNA did not fold into a GQ, probably due to the rigid nature of the loop position LNA-Ts. The LNA/DNA chimera, in which the tetrad
dGs have been substituted by LNA-G nucleotides, did fold into GQ; the CD spectra reflected parallel folding with a peak max. near $260 \mathrm{~nm}$. The hysteresis observed in the melting process referred to a nonunimolecular parallel scaffold. The anti LNA-Gs that substituted for syn dGs might have induced the formation of the parallel scaffold. The structure was very stable, and the $T_{m}$ value increased by $20^{\circ} \mathrm{C}$ relative to the wild type [154].

The htel-12 TAGGGTTAGGGT forms a parallel-antiparallel mixture of dimeric GQs; the latter antiparallel one contains both anti and syn nucleosides. Substitutions of the natural nucleotides by LNAs, which are restricted to the anti form, converted the antiparallel folds into parallel, which contains only anti nucleosides. The driving force of the conversion of topology is described as a combination of the $\mathrm{C}^{\prime}{ }^{\prime}$-endo puckering (Figure 9, 3) of the LNA nucleotides and their preference for the anti glycosidic conformation. In addition, the LNA-modified parallel GQs are significantly stabilized, by up to $11^{\circ} \mathrm{C}$ in their $T_{m}$ value, relative to their DNA counterparts [163]. LNA-G substituting for anti dG 
positions in the parallel-stranded $G Q$ of $\mathrm{T}_{2} \mathrm{G}_{3} \mathrm{TG}_{3} \mathrm{TG}_{3} \mathrm{TG}_{3} \mathrm{~T}$ and the hybrid-1-forming TT(htel-21)A strengthened the GQ, while substituting for syn $\mathrm{dG}$ positions reduced $T_{m}$ of the wild type or even disrupted the native GQ structure. Particularly large negative $\Delta T_{m}$ values were described for the parallelforming $\mathrm{GQ},-41$ to $-45^{\circ} \mathrm{C}$, from the $77^{\circ} \mathrm{C}$ of the native [148].

The tetramolecular parallel GQ $\left[\mathrm{TG}_{3} \mathrm{~T}\right]_{4}$ was stabilized by LNAs as much as by the ribo substitution [154]. The full LNA GQ showed the smallest hysteresis on reannealing, probably due to the rigid sugar part. The elevated stability was explained by entropy gains. An NMR study of two LNAmodified $\left[\mathrm{TG}_{4} \mathrm{~T}\right]_{4}$ GQs showed only local structural alterations, which were due to the $\mathrm{C}^{\prime}$-endo sugar pucker [155]. The crystal structure of an all-LNA-substituted tetramolecular parallel $\mathrm{GQ}$ formed from $\mathrm{TG}_{3} \mathrm{~T}$ was determined for the first time by Russo et al., refined at $1.7 \AA$ resolutions. A Ttetrad was formed at the $3^{\prime}$-end [164].

AS1411 is a GQ-forming aptamer capable of selectively entering cancer cells by nucleolin receptor-mediated uptake. Its internalization efficiency highly depends on the chemistry of the oligonucleotides. LNA substitutions were investigated with this GQ [165]. The formation of a GQ from a G-rich 25-mer section of the vascular endothelial growth factor aptamer was highly facilitated by LNA modifications. $T_{m}$ of $\sim 40^{\circ} \mathrm{C}$ for the unmodified GQ was raised to $\sim 52^{\circ} \mathrm{C}$ and $\sim 46^{\circ} \mathrm{C}$, respectively, in the two modified structures studied [166]. A series of interesting $3^{\prime}$-end-modified (capped) LNA analogs have been prepared by Kasahara et al. [167], in which the $2^{\prime}, 4^{\prime}$-methylene bridge was replaced by $-\mathrm{CH}_{2} \mathrm{OCH}_{2^{-}}$, $-\mathrm{NHCH}_{2}-$, and - $\mathrm{CH}(\mathrm{Ph}) \mathrm{OCH}_{2}$ - groups. The bridged nucleosides increased the resistance against nucleases in human serum of the TBA analogs, and the binding abilities were not affected by these modifications. Thermal stabilities were not published. Hotoda's anti-HIV-I oligonucleotide, the tetramolecular parallel GQ-forming TGGGAG's thermal stability, was significantly increased by LNA modifications. The unmodified had a $T_{1 / 2}$ value of $55^{\circ} \mathrm{C}$ while $T_{1 / 2}$ for TGGGAG was $75^{\circ} \mathrm{C}$, where the bold-underlined Gs were LNA-Gs. The LNA modification highly enhanced the anti-HIV-1 activity of the Hotoda GQ [168]. The U nucleosides of the natural RNA sequence (uuaggg) ${ }_{n}$ forming the TERRA GQ were also replaced by LNA and 2' -O-methyl ribonucleoside analogs to study the protein recognition of the loops ribose moieties. Stability data have not been published [169].

Unlocked Ribonucleosides (UNA). The UNA abbreviation stands for unlocked nucleic acid. In the unlocked nucleosides, the furanose ring's $\mathrm{C} 2^{\prime}-\mathrm{C} 3^{\prime}$ bond is missing; it is thus a $2^{\prime}$ $3^{\prime}$-acyclic-rN, a ribonucleoside analog (Figure 10, 2), which is often abbreviated as, for example, $\mathrm{uG}$ or $\mathrm{uU}$. These types of nucleosides are flexible ribo derivatives. The positional effects of single unlocked nucleosides, the $2^{\prime}-3^{\prime}$-acyclic-rG and -rU, were described with TBA GQs. Replacement of T by $\mathrm{uU}$ in loop positions 3,7 , and 12 , one in each of the three loops, proved stabilizing for the TBA GQ. $\mathrm{uU}$ in the other three T positions destabilized the GQ [170]. Uracil in the loops has been described to stabilize the TBA GQ, contrary to the duplexes, by $-0.16 \mathrm{kcal} / \mathrm{mol}$ each [1]; thus, with the $\mathrm{uU}$ nucleosides, this effect must have contributed to the stabilization. CD spectra of GQs with $\mathrm{uU}$ in positions 3, 7 , and 12 were the same as that of the unmodified TBA GQ. $\mathrm{uU}$ in the other loop positions changed the spectra by significantly lowering the amplitudes and causing band shifts. Incorporation of $\mathrm{uG}$ in any tetrad position either destabilized the GQ or hindered the formation of it. The GQ with a uU in position 7 proved to be the only one with more effectiveness in blood clotting than the unmodified GQ. The influence of a single $\mathrm{uU}$ and $2^{\prime}$-C-piperazino-uU residues (Figure 10, 4) incorporated into several positions of the TBA DNA was studied by Jensen et al. [171], who arrived at very similar conclusions as did Pasternak et al. [170]. The 2' -C-piperazino$\mathrm{uU}$ more efficiently stabilized the GQ structure than the $\mathrm{uU}$ and increased the thermal stability of the native TBA GQ by $2-3^{\circ} \mathrm{C}$ in a position-dependent manner. GQ topology and molecularity were retained. The presence of $\mathrm{uU}$ in positions U3, U7, and U12 resulted in the highest stabilization of the GQ. On the contrary, the largest destabilization mounting to $-15^{\circ} \mathrm{C}$ was observed when $\mathrm{uU}$ residues were placed in positions U7, G8, and U9. Kinetic studies indicated no strict correlation between thermodynamic stability and the binding affinity to thrombin. Most variants studied bound to thrombin, albeit with decreased affinity related to the wildtype TBA GQ [171]. A double modification study of the TBA with an UNA analog, the unlocked 4-thiouridine, the $\mathrm{s}^{4} \mathrm{uU}$ (Figure 10,3), has also been published. The analog in all possible positions of the three edgewise loops produced negative Gibbs' free energy indicating that at physiological temperature the predominant species were the folded GQ forms. However, most TBA variants were less stable than the unmodified GQ by $0.9-1.0 \mathrm{kcal} / \mathrm{mol}$. $\mathrm{s}^{4} \mathrm{uU}$ at positions 3 and 12 did not influence the thermodynamic stability, whereas, at position 7 , it increased it by $0.34 \mathrm{kcal} / \mathrm{mol}$. In contrast, the ribo 4-thiouridine introduced into positions 3, 7, 9, and 12 stabilized TBA GQ by $0.31-0.53 \mathrm{kcal} / \mathrm{mol}$ and was usually more stabilizing/less destabilizing than the $s^{4} \mathrm{uU}$. The modified TBAs retained the antiparallel GQ topology of the native GQ. Thrombin clotting time studies revealed that TBA modified with $s^{4} \mathrm{uU}$ at position 7 possessed high anticoagulant activities and the modified aptamer was a potent inhibitor of fibrin-clot formation [172]. Unlocked uA, $\mathrm{uT}$, or $\mathrm{uC}$ was substituted for the single-base diagonal loop and $u G$ for tetrad $G$ s in the $G Q$ of $G_{3} T_{5} G_{3} A$ (or $\left.T, C\right) G_{3} T_{5} G_{3}$. The loop (A, T, or C) modifications stabilized the GQ by $3-7^{\circ} \mathrm{C}$ in $T_{m}$, and the uA had the greatest effect. Stabilization was explained by the flexibility of the unlocked nucleoside, which could ease the tension that might exist in a single-base diagonal loop. Contrary to the loop substitutions, the uGs led to significant destabilization of the GQ. The $\mathrm{uG}$ in the middle tetrad caused the largest effect, and $\Delta T_{m}$ was $-17.6^{\circ} \mathrm{C}$. Flexibility of the sugar moiety did not prove to be a beneficial structural motif in the G-tetrads. The unlocked nucleosides caused transition from the antiparallel to hybrid-type fold in some of these GQ analogs [173]. Thymine glycol, also an acyclic analog of dT, (S)-GNA-T (Figure 10, 5), was substituted for loop T position of the TBA GQ. The analog destabilized the TBA in positions in T4,T9 by $9-13^{\circ} \mathrm{C}$, and stabilized it in $\mathrm{T} 7$ by $5.2^{\circ} \mathrm{C}$. With double substitutions in T7,9, T3,12, 
and T4,T13, the TBA GQ was destabilized by $2-12^{\circ} \mathrm{C}$ [94]. Aaldering et al. [57] reported on the impact of $\mathrm{uU}$ and the $3^{\prime}$-amino-uU on the structural dynamics and stability of TBA GQ by substituting them for the (nonstacking) loops T3, T7, and T12. While the $\mathrm{uU}$ enhanced the thermal stability of the unmodified GQ $\left(T_{m}: 49^{\circ} \mathrm{C}\right)$ at all three T positions, by $2-5^{\circ} \mathrm{C}$, the $3^{\prime}$-amino derivative decreased it by $2-7^{\circ} \mathrm{C}$. As the amino group replaced the $3^{\prime}-\mathrm{OH}$, the internucleotide linkage at this site was $2^{\prime}-5^{\prime}$ instead of the natural $3^{\prime}-5^{\prime}$ (this modification of the backbone is named isoDNA; see also later). The authors suggested that the altered backbone caused the destabilization effect with the $3^{\prime}$-amino modifications. On the other hand, none of the loop substitutions changed the topology of the native TBA GQ (CD peak max. at $295 \mathrm{~nm}$ and negative peak at $265 \mathrm{~nm}$ ), and both the unlocked uridine analogs improved the thrombin clotting time [57].

$\alpha$-Anomeric Sugars. $\alpha$-Nucleosides differ from the natural $\beta$-ones by the inversion of the configuration at the $\mathrm{C}\left(1^{\prime}\right)$ anomeric position of the furanose ring (Figure 10, 6) (for a review, see [174]). The $T_{2} G_{4} T_{2}$ that assembles into a parallel-stranded tetramolecular GQ was found to be an inhibitor of HIV infection, especially if the phosphodiesters internucleotide linkages were replaced with P-S linkages (see Section 3.3), and its $\mathrm{IC}_{50}$ value was $0.3 \mu \mathrm{M}$ [175]. When the same oligodeoxynucleotide was built from $\alpha$-nucleosides, the $\alpha\left(\mathrm{T}_{2} \mathrm{G}_{4} \mathrm{~T}_{2}\right)$, also with P-S linkages, a similar anti-HIV activity was observed, and the $\mathrm{IC}_{50}$ value was $0.5 \mu \mathrm{M}$ [176]. Since $\alpha$-oligonucleotides are nuclease-resistant [174], it was claimed that the P-S backbone is mechanistically required for antiviral activity of this oligonucleotide. It was, therefore, suggested that PS-ODN interacts with the highly cationic V3 loop. $\alpha$-Nucleosides, which cannot adopt syn glycosidic conformation [177], were substituted for Ts of the loops and dGs of tetrads of the native TBA GQ. Depending on the location of substitution, the GQ was either stabilized or destabilized by the $\alpha$-Ts in the loops. Significant stabilization was observed for the anomeric modification of TT loops at T4 and T13 (Figure 2). The T-substitutions did not change the antiparallel topology. The $\alpha$-dGs, however, either prevented the GQ assembly or induced rearrangement of the topology. Replacement of all natural nucleotides with the anomeric ones also resulted in random coil. The anticoagulant properties of the chimeric aptamers were retained only with intact TT loops. On the contrary, modification of the TGT loop only substantially increased the nuclease resistance of the chimeric aptamer without notable disturbance of its anticoagulant activity [178].

L-Nucleosides. L-Nucleosides (Figure 11, 1) are mirror images of the D-counterparts. Zintevir is a 17-mer DNA that assembles into a GQ of intramolecular parallel topology due to the single-base loop(s). This GQ shows strong anti-HIV-1 activity. The GQ from its L-sugar enantiomer building blocks, the L-GTGGTGGGTGGGTGGGT, showed comparable antiviral activity to Zintevir. Its CD spectrum was a mirror image and completely symmetrical to that of the D-GQ and thermal stability of the L-analog was very similar to that of the D [179]. The L-analog of the tetramolecular parallel GQ-forming
$\mathrm{TG}_{4} \mathrm{~T}$ was also synthesized later [180]. CD spectrum of $\mathrm{L}-$ $\left[\mathrm{TG}_{4} \mathrm{~T}\right]_{4}$ was again the inversion of the D-scaffold and thermal stabilities $\left(T_{1 / 2}\right)$ were identical within the experimental error, $57^{\circ} \mathrm{C}$ for the $\mathrm{D}$ - and $58^{\circ} \mathrm{C}$ for the L-structure. Thermal difference UV spectra, diagnostic of GQ formation, were nearly superimposable. Heterochiral or D/L-oligonucleotide chimeras based on $\mathrm{TG}_{4} \mathrm{~T}$ have been prepared by Antonella Virgilio et al. [181]. These could form either right-handed D- or left-handed L-GQs, depending on the composition and sequence. The $T_{D} G_{D} G_{L} G_{L} G_{D} T_{D}$ formed left-handed topology even if it was mainly composed of $\mathrm{D}$-nucleotides. L-Nucleotides were also incorporated into the 15-mer TBA DNA. All TBA sequences that contained various numbers of L-nucleotides were able to fold into the chair-type antiparallel GQs, similar to the wild type. Thermal stability of the full-L GQ was the same as that of the D; only the heterochiral structures were generally less stable than full chirals [182]. L-Nucleotides were also combined with polarity inversion sites. Virgilio et al. [183] prepared the heterochiral oligodeoxynucleotides $5^{\prime}-\mathrm{T}_{\mathrm{D}} \mathrm{G}_{\mathrm{D}} \mathrm{G}_{\mathrm{D}}-3^{\prime}-3^{\prime}-\mathrm{G}_{\mathrm{L}} \mathrm{G}_{\mathrm{L}} \mathrm{T}_{\mathrm{L}}$ $5^{\prime}$ (L33) and the $3^{\prime}-\mathrm{T}_{\mathrm{D}} \mathrm{G}_{\mathrm{D}} \mathrm{G}_{\mathrm{D}}-5^{\prime}-5^{\prime}-\mathrm{G}_{\mathrm{L}} \mathrm{G}_{\mathrm{L}} \mathrm{T}_{\mathrm{L}}-3^{\prime}$ (L55). Both formed $G Q$ structures and data indicated the presence of enantiomeric left- and right-handed GQ helices. In the case of L55, NMR experiments pointed to an unusual equilibrium between them. TBA sequence was also derivatized, like the $5^{\prime}-\mathrm{G}_{\mathrm{L}} \mathrm{G}_{\mathrm{L}}-3^{\prime}-3^{\prime}-\mathrm{TT}-5^{\prime}-5^{\prime}-\mathrm{G}_{\mathrm{L}} \mathrm{G}_{\mathrm{L}} \mathrm{T}_{\mathrm{L}} \mathrm{G}_{\mathrm{L}} \mathrm{T}_{\mathrm{L}} \mathrm{G}_{\mathrm{L}} \mathrm{G}_{\mathrm{L}}-3^{\prime}-3^{\prime}-$ TT $-5^{\prime}-5^{\prime}-\mathrm{G}_{\mathrm{L}} \mathrm{G}_{\mathrm{L}}-3^{\prime}$. Interestingly, all modified oligodeoxynucleotides folded into the two-tetrad, chair-like antiparallel topology. Thermal stabilities depended on the position of the L-nucleotides and the inversion sites [184]. Aviñó et al. substituted the TBA thymines with L-Ts in loop positions 3, 4 , and 7 , and all destabilized the GQ by 0.7 to $4^{\circ} \mathrm{C}$ [94].

GQs and i-motif (C-)GQs have also been built from $\beta$-L-ribonucleotides. Thermal stability of the $\beta$-L$\mathrm{r}$ (AGGAGGAGGAGGA) GQ and the $\beta$-L-r $\left(\mathrm{C}_{3} \mathrm{UC}_{3} \mathrm{U}_{4} \mathrm{C}_{3} \mathrm{UC}_{3}\right)$ i-motif in $100 \mathrm{mM} \mathrm{KCl}$ and $\mathrm{pH} 7$ and $\mathrm{pH} 3.4$, respectively, was the same or very similar to those of the $\beta$-D-ribo, that is, to the natural GQs, near $73^{\circ} \mathrm{C}$ and $33^{\circ} \mathrm{C}$, respectively [185]. A recent paper also dealt with anomeric L-nucleic acids by examining the structural features of a mixed L-RNA/L-DNA GQ aptamer [186]. When L-nucleotides were incorporated into a DNAzyme sequence, which could fold into an intramolecular L-GQ, the resulting L-DNAzyme exhibited peroxidase catalytic activity [187].

Isonucleosides. Instead of the natural $\mathrm{Cl}^{\prime}-\mathrm{N} 1 \mathrm{~N}$-glycosidic linkage, the $\mathrm{C}^{\prime}{ }^{\prime} \mathrm{N} 1$ linkage-containing isothymidines (Figure 11, 2, 3) were introduced into the loop section of the TBA DNA, in which the isonucleosides were also in either the natural D or the mirror image L stereo configuration, the $\mathrm{D}$ - and L-isothymidines [188]. Incorporation of D-iso $\mathrm{T}$ into positions T3 and T12 of the two TT loops and T7 of the TGT loop (Figure 2) increased the thermal stability of native TBA GQ by 0.9 to $5.4^{\circ} \mathrm{C}$. The mirror image L-iso T in positions of T3, T12, and T9 (TGT loop) also increased the stability by 2.5 to $5.1^{\circ} \mathrm{C}$. Substitution of all other T positions by either $\mathrm{D}$ - or L-iso T resulted in significant destabilization of the scaffold, by -3.3 to $-17.5^{\circ} \mathrm{C}$. Mixed double modifications were also tested in this study from which a few also stabilized, while others destabilized the native TBA GQ [188]. 


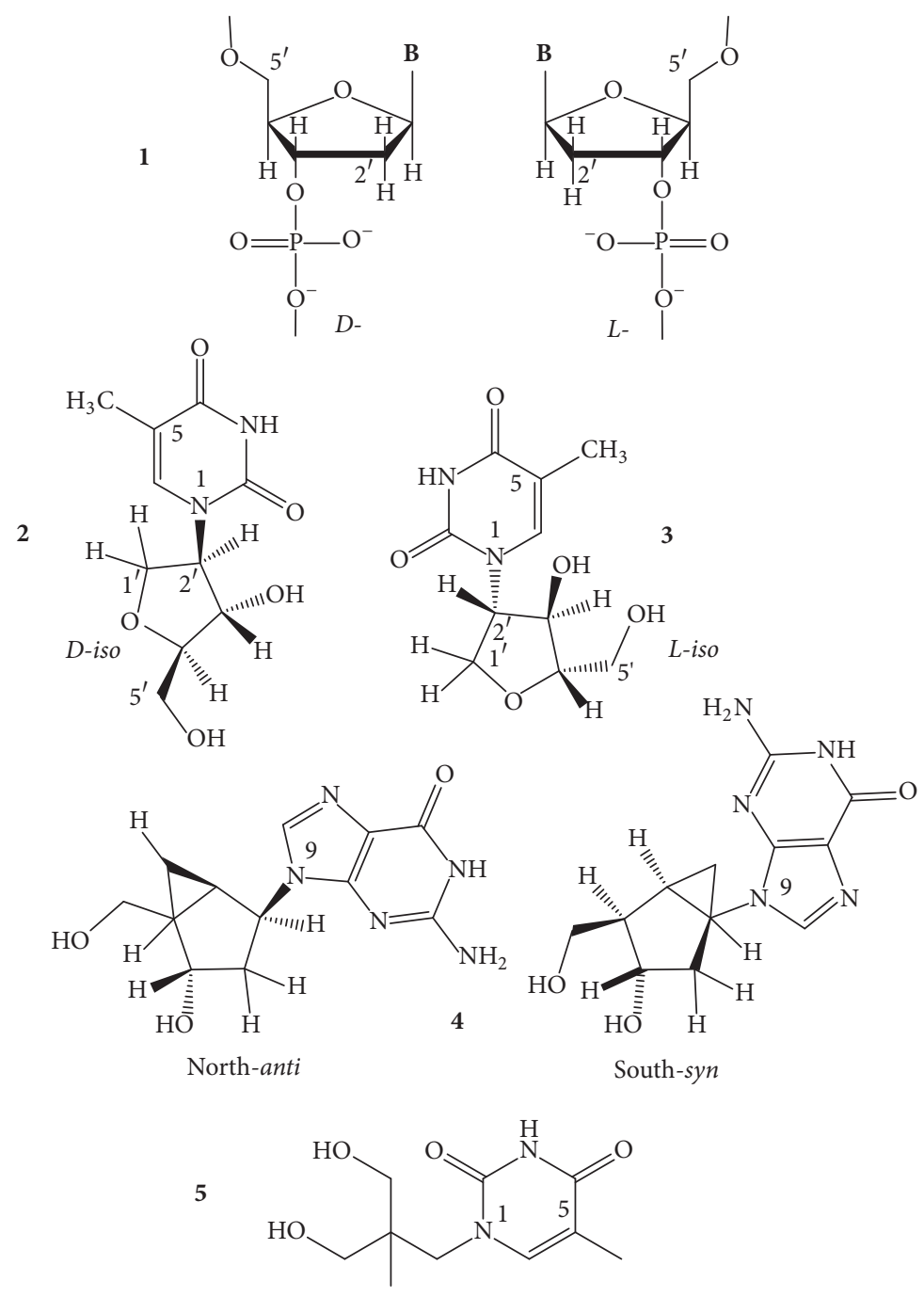

Figure 11: The mirror image D- and L-2'-deoxynucleosides (1); the D- (2) and L-iso-thymidines (3); the anti north- and the syn southbicyclo[3.1.0]hexane-dGs (4); and an acyclic thymine analog, the $\mathrm{N}^{1}$-(3-hydroxy-2-hydroxymethyl-2-methylpropyl)-thymine (5).

Other Modifications of the Sugar Moiety. Besides LNA, another conformationally rigid sugar analog of dG, the syn and anti, north- and south-bicyclo[3.1.0]hexane-dGs (Figure 11, 4) were substituted for G14 and G15 of the TBA GQ $[189,190]$. Substitution of position 14 did not change the stability and topology of the wild-type TBA GQ; substitution of position 15, however, caused a strong destabilization. Coppola et al. [191] substituted TBA's thymidines, one at a time, with acyclic thymine analogs, namely, $\mathrm{N}^{1}$-(3-hydroxy-2-hydroxymethyl-2-methylpropyl)-thymine (Figure 11, 5), a sort of unlocked nucleotide. Nearly all TBA analogs were able to fold into similar GQ structure as the native DNA did. Substitution of loops T3, T7, and T12 by the acyclic-Ts slightly stabilized, by $1-4^{\circ} \mathrm{C}$, the GQ, relative to the unmodified TBA, whereas that of $\mathrm{T} 4$ and $\mathrm{T} 13$ slightly destabilized, by $3^{\circ} \mathrm{C}$, and that of the $\mathrm{T} 9$ significantly reduced the thermal stability of the unmodified TBA, from $50^{\circ} \mathrm{C}$ to $39^{\circ} \mathrm{C}$ [191].

Multiple Modifications of the Sugar Part. When hemin interacts with certain GQs, the complex will exhibit peroxidase activity. These hemin-binding GQs have recently emerged as important peroxidase mimicking DNAzymes and have been utilized in bioanalytical applications. Li et al. [192] studied the effect of multiple chemical modifications of the sugar part: $2^{\prime}$-O-methyl-, L-nucleotides and LNAs; furthermore, the backbone was also modified with phosphorothioates (P-S). Three GQs with known high peroxidase activity were selected for the experiments, the PS2.M, $\mathrm{GTG}_{3} \mathrm{TAG}_{3} \mathrm{CG}_{3} \mathrm{~T}_{2} \mathrm{G}_{2}$, the c$\mathrm{Myc}, \mathrm{TGAG}_{3} \mathrm{TG}_{4} \mathrm{AG}_{3} \mathrm{TG}_{4} \mathrm{~A}_{2}$, and the EAD2, $\mathrm{CTG}_{3}\left(\mathrm{AG}_{3}\right)_{3} \mathrm{~A}$. Results indicated that the $2^{\prime}$-O-methyl modification facilitated the formation of perfectly compacted parallel structures with the highest thermal stabilities among the unmodified and modified GQs and with significantly promoted peroxidase activity of GQ based DNAzymes. The LNA, L-, and the P-S backbone modifications reduced the stability of wild types [192].

GRO29A is a GQ-forming, growth-inhibitory oligonucleotide, whose sequence is $T_{3} G_{2} T_{2} T_{2} T_{2} T_{2} T_{2} G_{2} T G_{2} T$ $\mathrm{G}_{2} \mathrm{TG}_{2}$ [193]. Several sugar and backbone-modified analogs 
of GRO29A have been prepared by incorporating $2^{\prime}$-Omethylribo-, mixed $2^{\prime}$-deoxy, and $2^{\prime}$-O-methylribo nucleosides and backbone-modified P-S analogs. The full P-S, the $2^{\prime}$-O-methyluracil (RNA) $2^{\prime}$-deoxyguanosine-containing chimeric and the full $2^{\prime}$-O-methylribo analogs increased the thermal stability of the GQ of the $3^{\prime}$-aminoalkyl-modified GRO29A, while the other analogs decreased it or no thermal profiles were detected. The P-S and chimeric analogs were found to significantly inhibit proliferation of a number of tumor cell lines, but the $2^{\prime}$-O-methyl RNA analog had no significant effect. Based on molecular modeling, it was concluded that the inactivity of the latter was due to the differences in the groove structure of the GQ, compared to the other's [194].

The effects of multiple substitutions of the TBA DNA by LNA and 2'-O-methyl analogs of G (LNA-G and om $^{2 \prime} \mathrm{G}$ ) on the formation and topology of the TBA GQs have been studied. Results showed that when two or more Gs were substituted with LNA-G or om ${ }^{2 \prime}$, the 15-mer TBA oligonucleotide remained unstructured in $50 \mathrm{mM} \mathrm{K}^{+}$. The native TBA GQ was unstructured in $50 \mathrm{mM} \mathrm{Ca}^{2+}$, and interestingly, four or more LNA-G or om $^{2 \prime} \mathrm{G}$ substitutions induced the formation of parallel GQs, which were dimeric forms [195].

Chain Terminal Modifications. "Sweetening" the TBA GQ, that is, attaching various $5^{\prime}$-terminal sugar derivatives to the 15-mer TBA DNA, led to the reduction of the thermal stability of the native GQ by 4.5 to $10.1^{\circ} \mathrm{C}$. The sugar moieties were connected to the $5^{\prime}$-dG through a phosphodiester group, for example, the $\beta$-D-glucose- $\mathrm{C}_{2}-\mathrm{OPO}_{2}-5^{\prime}$ TBA. $\beta$-D-Galactose, -maltose, -lactose, -cellobiose, and $\beta$-Lfucose were also appended. Compared to the $\mathrm{HO}-\mathrm{C}_{2}-\mathrm{OPO}_{2}$ derivatized TBA GQ, not the native TBA GQ, there was a slight increase in the $T_{m}$ values, 0.7 to $2.8^{\circ} \mathrm{C}$, with the added sugar moieties. Attaching carbohydrates to the $5^{\prime}$-end of the telomeric oligonucleotide of TAGGGTTAGGGT could alter the folding topology of the parent GQ. The sugar moieties can well stack with the $5^{\prime}$-G-tetrad [196]. Four cationic amino acids, lysine, ornithine, homo-arginine, and arginine, were covalently attached to the $3^{\prime}$-end of the tetrahymena telomeric repeat DNA sequence $\mathrm{TG}_{4} \mathrm{~T}$ [197]. The cationic residues did not affect the formation of parallel tetramolecular GQ structures, and the $3^{\prime}$-conjugations increased the thermal stability of $\left[\mathrm{TG}_{4} \mathrm{~T}\right]_{4}$ by 3 to $6.4^{\circ} \mathrm{C}$ in $T_{m}$ in $0.11 \mathrm{M}$ $\mathrm{Na}^{+}$[197]. Thermal stabilization by several other terminal modifications of tetramolecular GQs has been reviewed by Doluca and coworkers [4] and more recently by Prokofieva et al. [15] who analyzed the GQ-forming chain end-modified oligonucleotide aptamers, primarily the Hotoda TGGGAG analogs, in connection with their anti-HIV activity. The anti-HIV activity of GQs is exerted via preventing the viral entry into the target cells by binding to the gp120 surface glycoprotein of the virus and by targeting the viral integrase or the viral reverse transcriptase within the HIV, as it was reviewed by Musumeci et al. [16]. The $5^{\prime}$-terminal modifications were the dimethoxytrityl, 3,4-dibenzyloxybenzyl, tert-butyldiphenylsilyl, and other aromatic groups. $3^{\prime}$-End modifications also enhanced the aptamer activity, such as the 2-hydroxyethylphosphate, glucose, or the mannose moieties. LNA-G at certain tetrad positions also resulted in potent activity with increased thermal stability of the GQ. No strict correlation was found between the stability and the aptamer activity of the GQs. Terminal modifications, especially the hydrophobic groups, can increase the thermal stability of GQs. The backbone modifications, such as the thiophosphoryl (P-S) internucleotide linkages, increased the resistance to cellular nucleases, which is important for the aptamer activity [15].

\subsection{Phosphodiester and Sugar-Phosphate Backbone Analogs}

Phosphorothioates (P-S). A sulfur atom replaces one of the nonbonded oxygen atoms of the phosphodiester (P-O) internucleotide linkage (Figure 12, 1) in the phosphorothioate (P-S) linkage (Figure 12, 2). The sulfur can be in $R_{P}$ or $S_{P}$ stereo configuration, bringing in the chirality problem. In most cases, however, the isomers are not separated after the synthesis. Sulfur has a larger size than the oxygen and is less hydrophilic. If the $\mathrm{P}-\mathrm{O}$ linkages are replaced with the random stereo mixture P-S linkages in a DNA or RNA strand, its duplex with an unmodified complementary strand is thermodynamically destabilized relative to the full $\mathrm{P}-\mathrm{O}$ duplex. If the pure stereo full- $\mathrm{R}_{\mathrm{p}}$ or full- $\mathrm{S}_{\mathrm{P}}$ isomeric strands are compared, the negative effect of $S_{P}$ isomers is larger than the full- $R_{P}$ strands in their duplexes [198]. However, the P-S strands become highly resistant against cellular nucleases, and this property made the P-S oligonucleotides the first generation of therapeutically applicable antisense oligonucleotides, as reviewed by Eckstein [199]. As for the GQs, when all P-O linkages were replaced by P-S in the tetramolecular parallel $\left[\mathrm{TG}_{4} \mathrm{~T}\right]_{4}$, in the 12-mer bimolecular $\left[\mathrm{G}_{4} \mathrm{~T}_{4} \mathrm{G}_{4}\right]_{2} \mathrm{GQs}$ in $\mathrm{Na}^{+}$and in the intramolecular antiparallel TBA GQ in $\mathrm{K}^{+}$, the GQs were all destabilized. The P$S$ substitution of the TBA was studied in more detail by Zaitseva et al. [200] and Prokofjeva et al. [15]. The CD spectrum of the unmodified TBA GQ was not changed by P$\mathrm{S}$ modifications. Presence of a single P-S bond between two G-quartet planes (the G-G bond) led to a significant decrease in GQ thermostability, from $52^{\circ} \mathrm{C}$ to $45^{\circ} \mathrm{C}$ in $100 \mathrm{mM} \mathrm{K}^{+}$. On the contrary, modifications in the loop sequences (T-T bonds) either did not alter or stabilized the structure [15].

Interestingly, the P-S substitution had no effect in $\mathrm{Na}^{+}$ and increased the stability in $\mathrm{K}^{+}$of the intramolecular GQs built from the 18-mer AGG(TTAGG) ${ }_{3}$ and the $\mathrm{AG}_{3}\left(\mathrm{TTAG}_{3}\right)_{3}$ [1]. Another sequence, the $T_{2} G_{4} T_{2}$, forms a parallel-stranded $\mathrm{GQ}$, which has antiviral activity. The tetramolecular structure binds to the human immunodeficiency virus envelope protein gpl20 at the V3 loop and inhibits both the cell-to-cell and virus-to-cell infection. The P-S analog of $T_{2} G_{4} T_{2}$ was found to be a more potential inhibitor of HIV infection, in vitro $[175,176]$. Site-directed spin labeling technique was used for investigating GQ conformations by Zhang et al. [201]. Double nitroxide labels were attached to the sulfur atom of a P-S linkage at G3 and G15, the G4 and G15, and the G9 and G15 of the A(htel-21) oligonucleotide. The labels only marginally impacted the folding feature of the GQs and could adequately 

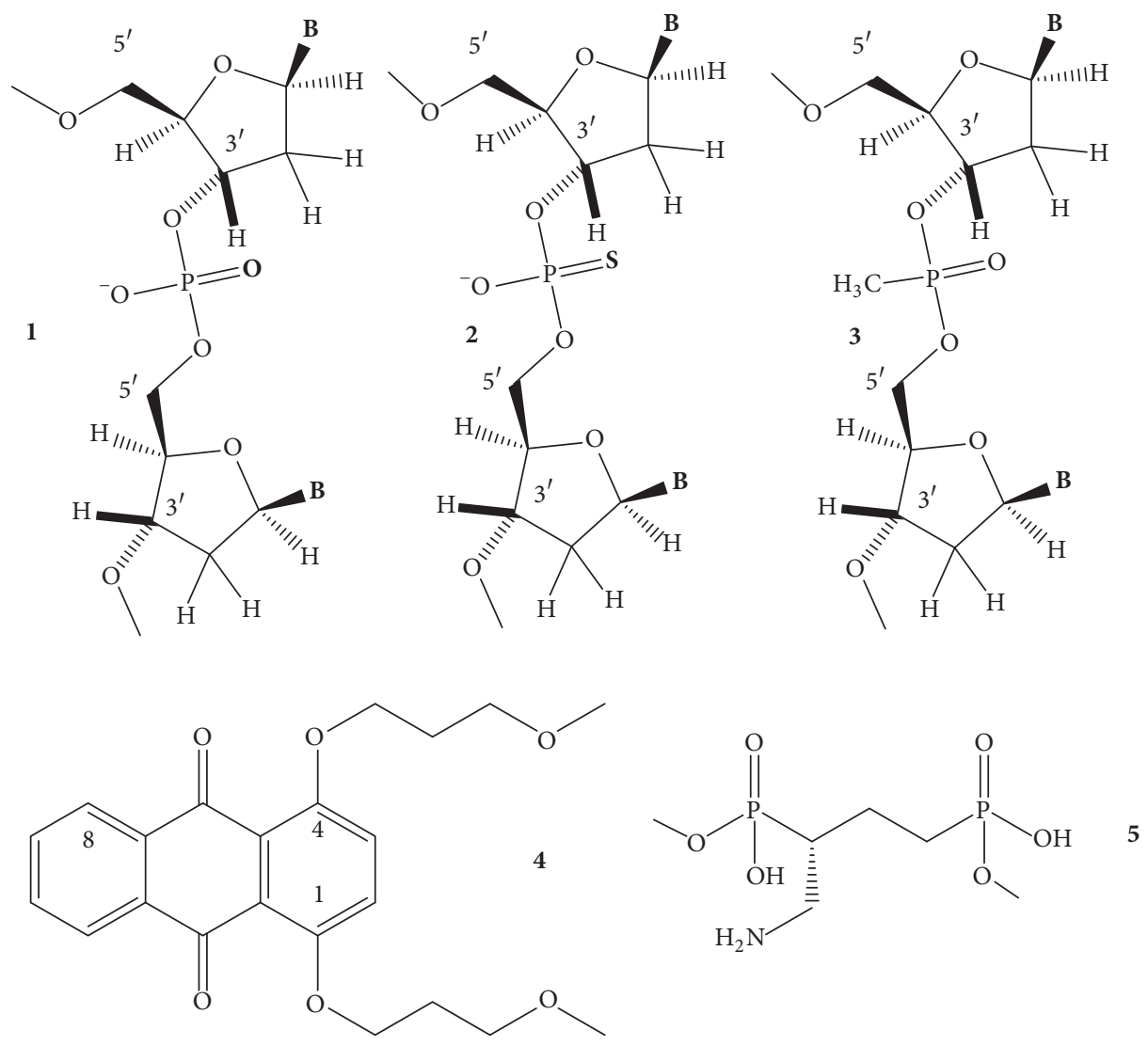

6<smiles>c1cc2ccc3cccc4ccc(c1)c2c34</smiles>

FIGURE 12: $2^{\prime}$-Deoxydinucleotides connected by the natural phosphodiester (P-O) (1); the thiophosphoryl or phosphorothioate (P-S) (2) and the nonionic methylphosphonate (P-Me) linkages (3); the 1,4-disubstituted dihydroxyanthraquinone linker (4); the acyclic (R)-4aminobutane-1,3-diol phosphodiester backbone (5); and the pyrene molecule used for loop (6).

distinguish between different topological conformations of the A(htel-21) GQ.

Inversion of Polarity Sites. In natural nucleic acids, the nucleotides are connected via $3^{\prime}-5^{\prime}$ phosphodiester linkages. When this is changed to $3^{\prime}-3^{\prime}$ or $5^{\prime}-5^{\prime}$ linkages, it is called inversion of polarity. In GQ-forming oligonucleotides, such sites can be introduced at three different locations: in non-G-tracts, in G-tracts, and between a non-G-tract and a G-tract. The polarity inversion modifications in GQs have been reviewed by Virgilio et al. [9]. The inversion polarity site-containing GQs, a new class of GQs, have been introduced in 2005, and the first such structures contained $3^{\prime}-3^{\prime}$ or $5^{\prime}-5^{\prime}$ inversion sites in the G-tract [202]. Later, a library of 14 inversed-polarity site-containing TBA GQs have been prepared: seven with $5^{\prime}-5^{\prime}$ and seven with $3^{\prime}-3^{\prime}$ inversions. The former started with the $3^{\prime}$ G5 ${ }^{\prime}-5^{\prime}$ GTTGGTGTGGTTGG- $3^{\prime}$ and continued up in the sequence up to $3^{\prime}$-GGTTGGT5 $5^{\prime}-5^{\prime}$ GTGGTTGG- $3^{\prime}$. The latter group started in $5^{\prime}-\mathrm{G} 3^{\prime}-3^{\prime}$ GTTGGTGTGGTTGG-5 $5^{\prime}$ and continued up to $5^{\prime}$-GGTTGGT3'-3'GTGGTTGG-5'. The structures were characterized by NMR spectroscopy. The $5^{\prime}-5^{\prime}$ inversion site in the G-tract prevented the formation of stable GQ structures [203]. Another study showed that, among the $\mathrm{T}^{\prime}-5^{\prime} \mathrm{T}$ series, the $3^{\prime}-\mathrm{GGT} 5^{\prime}$ $5^{\prime}$ TGGTGTGGTTGG- $3^{\prime}$ sequence folded into an unusual structure adopting three strands parallel to each other and only one strand oriented in the opposite manner. This modified TBA was more stable than its unmodified counterpart and showed a high thrombin affinity. $T_{m}$ of the wild-type GQ was $53^{\circ} \mathrm{C}$ and that of the inversed-polarity analog was $57.9^{\circ} \mathrm{C}[204,205]$. GQ of the $3^{\prime}-\mathrm{TG}^{\prime}-5^{\prime} \mathrm{GGGT} 3^{\prime}$ (QS55) and the $5^{\prime} \mathrm{TG} 3^{\prime}-3^{\prime}$ GGGT5' (QS33) formed different topologies. NMR analysis revealed that the latter formed a parallel-like fourfold symmetric GQ, while the former possessed twofold symmetry and was characterized as a tetramer antiparallel GQ embedded between two parallel 
tracts. The inversed-polarity-containing GQs had reduced thermal stability relative to the native fourfold GQ. $T_{m}$ values of $65^{\circ} \mathrm{C}, 47^{\circ} \mathrm{C}$, and $53^{\circ} \mathrm{C}$ were measured for the $[\mathrm{TGGGGT}]_{4}$, the $\left[5^{\prime} \mathrm{TG} 3^{\prime}-3^{\prime} \mathrm{GGGT} 5^{\prime}\right]_{4}$, and the $\left[3^{\prime} \mathrm{TG} 5^{\prime}-\right.$ $\left.5^{\prime} \mathrm{GGGT}^{\prime}\right]_{4}$, respectively [206]. NMR and CD studies of two polarity-inversed GQs, named Q33 and Q55, formed by the oligodeoxynucleotides $5^{\prime}$-TGG $3^{\prime}-3^{\prime}$ GGT- $5^{\prime}$ and $3^{\prime}$ TGG5 $5^{\prime}-5^{\prime}$ GGT-3', respectively, had different arrangement of the glycosidic angles of the residues, which led to a different symmetry and different physical properties of the two GQs. Both GQs assumed right-handed conformation. The Q33 had all $\mathrm{G}$ residues in anti glycosidic conformation, while the Q55 had one G-quartet with all-syn residues. Interestingly, the Q55 was more stable by $25^{\circ} \mathrm{C}$ than the natural counterpart, whereas Q33 was a less stable structure $\left(T_{m}=52^{\circ} \mathrm{C}\right)$ [207]. Inversed-polarity sites were also introduced into the $\mathrm{TG}_{3} \mathrm{~T}$ oligonucleotide, which folds into $\left[\mathrm{TG}_{3} \mathrm{~T}\right]_{4}$ in $\mathrm{K}^{+}$solution [208]. The presence of these unnatural sites did not hinder the formation of tetramolecular GQs and in some cases increased the thermal stability of the unmodified one. $T_{m}$ of the GQ formed from the native sequence TGGGT was $45^{\circ} \mathrm{C}$, that of $5^{\prime} \mathrm{T} 3^{\prime}-3^{\prime} \mathrm{GGGT} 5^{\prime}$ was $44^{\circ} \mathrm{C}$; that of $5^{\prime} \mathrm{TG} 3^{\prime}-$ $3^{\prime} \mathrm{GGT} 5^{\prime}$ was $49^{\circ} \mathrm{C}$; that of $3^{\prime} \mathrm{T}^{\prime}-5^{\prime} \mathrm{GGGT} 3^{\prime}$ was $45^{\circ} \mathrm{C}$; and that of $3^{\prime} \mathrm{TG}^{\prime}-5^{\prime} \mathrm{GGT} 3^{\prime}$ was $72^{\circ} \mathrm{C}$ [205]. Virgilio's group has also studied $5^{\prime}$ TGGGGT3 $^{\prime}$ analogs that contained two or three $3^{\prime}-3^{\prime}$ or $5^{\prime}-5^{\prime}$ inversion sites in the G-run, namely, $5^{\prime} \mathrm{TG} 3^{\prime}-3^{\prime} \mathrm{G} 5^{\prime}-5^{\prime} \mathrm{GGT} 3^{\prime}, \quad 5^{\prime} \mathrm{TG} 3^{\prime}-3^{\prime} \mathrm{GG} 5^{\prime}-5^{\prime} \mathrm{GT} 3^{\prime}$, $5^{\prime} \mathrm{TGG} 3^{\prime}-3^{\prime} \mathrm{G} 5^{\prime}-5^{\prime} \mathrm{GT} 3^{\prime}, \quad 5^{\prime} \mathrm{TG} 3^{\prime}-3^{\prime} \mathrm{G} 5^{\prime}-5^{\prime} \mathrm{G} 3^{\prime}-3^{\prime} \mathrm{GT} 5^{\prime}$, and $3^{\prime} \mathrm{TG}^{\prime}-5^{\prime} \mathrm{G} 3^{\prime}-3^{\prime} \mathrm{G} 5^{\prime}-5^{\prime} \mathrm{GT} 3^{\prime}$ [209]. The modified sequences contained either no or only one natural $3^{\prime}-5^{\prime}$ linkage in the G-tract; notwithstanding, all analogs formed stable tetramolecular GQs. Results showed that the $3^{\prime}-3^{\prime}$ or $5^{\prime}-5^{\prime}$ inversion sites affected the glycosidic conformation of dGs and, consequently, also base stacking. This study allowed the authors to depict some generalizations concerning strand arrangements and the glycosidic conformational preference of residues adjacent to inverted polarity sites [209]. The inversion sites could increase the resistance of the TBA GQs against degradation by cellular $3^{\prime}$-exonucleases. Eight TBA analogs have been examined, which contained the inversion site at the $3^{\prime}$-end of the oligonucleotides that also contained an added natural base at the $3^{\prime}$. Some of them contained also a $5^{\prime}$-inversion site also with an added nucleotide. A few TBA analogs not only were much less sensitive towards various exonucleases but also were active aptamers and had elevated thermostability. The $5^{\prime}-\mathrm{G}_{2} \mathrm{~T}_{2} \mathrm{G}_{2} \mathrm{TGTG}_{2} \mathrm{~T}_{2} \mathrm{G}_{2}$ $3^{\prime}-3^{\prime}-\mathrm{A}$ was the most stable with a $\Delta T_{m}$ of $12^{\circ} \mathrm{C}$, and the A $-5^{\prime}-5^{\prime}-G_{2} T_{2} G_{2}$ TGTG $T_{2} \mathrm{~T}_{2}-3^{\prime}-3^{\prime}-\mathrm{T}$ was the least stable with a $\Delta T_{m}$ of $-1^{\circ} \mathrm{C}$, relative to the stability of the unmodified GQ. The modifications did not change the antiparallel, chair-type conformation characterizing the wild type [210].

Polarity inversion-site containing GQs proved more resistant to exonucleases than the wild types [210, 211], and some of them also showed antiproliferative activity [211]. GQ analogs have also been built from $\left(\mathrm{TG}_{2} \mathrm{~T}\right)_{4},\left(\mathrm{TG}_{3} \mathrm{~T}\right)_{4}$, and $\left(\mathrm{TG}_{4} \mathrm{~T}\right)_{4}$ that contained one $3^{\prime}-3^{\prime}$ and two $5^{\prime}-5^{\prime}$ inversion sites. The modified $\left(\mathrm{TG}_{3} \mathrm{~T}\right)_{4}$ and $\left(\mathrm{TG}_{4} \mathrm{~T}\right)_{4}$ formed very stable monomolecular parallel GQ structures characterized by three side loops containing the inversion of polarity sites.
Both contained an all-syn G-tetrad, while the other dGs adopted anti glycosidic conformations. Both GQs showed remarkable antiproliferative activity against cancer cell lines [211]. Oliviero et al. [212] published on polarity site inversion GQs with the aim of obtaining structurally homogenous DNA G-wire nanostructures, using $5^{\prime}$-CGGT-3'-3'-GGC$5^{\prime}$ sequences. An NMR study performed by Šket et al. on the tetramolecular GQs formed by $\mathrm{TG}_{3} \mathrm{~T}$ and its analogs containing a $5^{\prime}-5^{\prime}$ or $3^{\prime}-3^{\prime}$ inversion of polarity site, namely, the $3^{\prime} \mathrm{TG} 5^{\prime}-5^{\prime} \mathrm{G}_{2} \mathrm{~T} 3^{\prime}, 3^{\prime} \mathrm{T} 5^{\prime}-5^{\prime} \mathrm{G}_{3} \mathrm{~T} 3^{\prime}$, and the $5^{\prime} \mathrm{TG} 3^{\prime}-3^{\prime} \mathrm{G}_{2} \mathrm{~T} 5^{\prime}$, revealed that the parallel GQs had distinct cation-binding preferences [213]. When not only one or more inversion sites are introduced into an oligonucleotide but also the whole sequence is reversed from the $5^{\prime}-3^{\prime}$ direction to $3^{\prime}$ $5^{\prime}$, Marušič and Plavec [214] introduced the term sequence inversion or sequence. G-rich oligonucleotides of this full sequence inversion could assemble into GQs; however, both the thermal stability and the number of structures formed changed, as it was described for the natural $5^{\prime}-3^{\prime} 27-$ mer $\mathrm{G}_{3} \mathrm{TAG}_{3} \mathrm{CAG}_{4} \mathrm{ACACAG}_{3} \mathrm{TAG}_{3}$, a noncoding segment of the human papilloma virus (HPV) type 52, with its two truncated sequences. The reversed sequence of the $27-\mathrm{mer}$, illustrated as $5^{\prime}-3^{\prime}$, was the $\mathrm{G}_{3} \mathrm{ATG}_{3} \mathrm{ACACAG}_{4} \mathrm{ACG}_{3} \mathrm{ATG}_{3}$. For the three reversed sequence-GQs, the $T_{m}$ values were lower by 1 to $7^{\circ} \mathrm{C}$ in $\mathrm{K}^{+}$and 3 to $7^{\circ} \mathrm{C}$ in $\mathrm{Na}^{+}$. The inverted sequences showed completely different folding preferences from what the natural sequences did [214].

Another example for the inversed polarities is with hexaethylene-glycol (HEG). A nonnucleotide bridge connects two $\mathrm{TG}_{3} \mathrm{AG}$ oligonucleotides, whose connection was built with inversed polarities: $5^{\prime}-\mathrm{TG}_{3} \mathrm{AG} 3^{\prime}-\mathrm{p}-\mathrm{HEG}-\mathrm{p}-$ $3^{\prime} \mathrm{GAG}_{3} \mathrm{~T}-5^{\prime}$ and the $3^{\prime}-\mathrm{GAG}_{3} \mathrm{~T} 5^{\prime}-\mathrm{p}-\mathrm{HEG}-\mathrm{p}-5^{\prime} \mathrm{TG}_{3} \mathrm{AG}-3^{\prime}$. In $\mathrm{K}^{+}$solution, the molecules formed tetramolecular parallel GQs where the two HEG bridges formed two loops. The wild-type tetramolecular parallel GQ of $\mathrm{TG}_{3} \mathrm{AG}$ had a $T_{m}$ of $41.5^{\circ} \mathrm{C}$. The HEG GQs had much higher thermal stability: the first analog $76.0^{\circ} \mathrm{C}$ and the second $75.5^{\circ} \mathrm{C}$. The conjugated GQs exhibited elevated resistance in human serum and high or moderate anti-HIV-1 activity with low cytotoxicity. As a result, these conjugated hairpins represent the first active anti-HIV-1 bimolecular GQs based on the $\mathrm{TG}_{3} \mathrm{AG}$ sequence [215]. A doubly modified GQ containing polarity inversion has also been described: L-residues and inversion of polarity sites have been introduced into five TBA DNAs by Esposito et al. GQs of the all-L-TBA oligonucleotides as well as the all-L minus the first and third TT loops, which remained D-thymidines, folded into left-handed GQs, and, interestingly, thermal stability of the wild type was retained in both modified structures. Two mixed $\mathrm{L}-$ and inversed-polarity site sequences also formed left-handed GQs and their stabilities changed depending on the site of modification. The TBA analogs containing L-residues and inversion of polarity sites lost the anticoagulant activity but gained antiproliferative properties against two cancer cell lines [216]. In an NMR study, Esposito et al. used inversed-polarity-d(TGGGT)s, which also contained an AP (abasic) site, and revealed interesting changes of the anti and syn glycosidic conformation in the G-tetrads of the tetramolecular parallel GQs [217]. 
Nonnucleotide Backbone. 1,4-Dihydroxyanthraquinone and 1,8-dihydroxyanthraquinone linkers (Figure 12, 4) were substituted by Gouda et al. [218] for the loop T nucleotides of the TBA GQ. Single substitutions generally reduced the stability of native TBA $\left(T_{m} 50^{\circ} \mathrm{C}\right.$ in $\left.100 \mathrm{mM} \mathrm{K}^{+}\right)$. On the other hand, double substitutions by either linker led to stabilizations by 4 to $26^{\circ} \mathrm{C}$. The latter was achieved with the 1,4-disubstituted anthraquinone linker in positions $\mathrm{T} 4$ and $\mathrm{T} 13$ in the two lateral TT loops. The modifications retained the antiparallel conformations of the native TBA GQ. Majority of the anthraquinone-modified TBA GQs showed decreases in the clotting times, compared to TBA. The aptamers containing the 1,8-linker at G8 or T9 in the TGT loop had improved anticoagulant activity [218]. Gouda et al. [219] also replaced the TT and TGT loops of the TBA GQ with novel 1,5- and 2,6-disubstituted anthraquinones. Single substitutions destabilized, while anthraquinones in two TT loops led to $1-18^{\circ} \mathrm{C}$ increase in $T_{m}$ of the parent GQ without changing the original topology. TBA nucleotides have also been substituted with acyclic (R)-4-aminobutane1,3-diol phosphodiester backbone (Figure 12, 5). Substitution of T7 resulted in a slight increase in $T_{m}$ with no effect on conformation. Double substitutions of G5G6, or multiple T positions, resulted in significant decreases in the $T_{m}$ and the $\Delta G_{310}$ values [220]. One, two, and all three TTT loops were replaced by pyrene molecules (Figure 12, 6) of the 21-mer $\mathrm{G}_{3}\left(\mathrm{~T}_{3} \mathrm{G}_{3}\right)_{3}$ oligodeoxynucleotide by Rajagopal and Hariharan [221]. In $100 \mathrm{mM} \mathrm{KCl}$, the thermal stability of parent antiparallel/hybrid GQ (positive max. around $290 \mathrm{~nm}$ with a strong shoulder/peak near $260 \mathrm{~nm}$, negative max. close to $240 \mathrm{~nm}$ in its $\mathrm{CD}$ spectrum) of $70.2^{\circ} \mathrm{C}$ was not changed by a single pyrene backbone, $T_{m} 69.9^{\circ} \mathrm{C}$; however, the stability was increased to $74.5^{\circ} \mathrm{C}$ and $87.2^{\circ} \mathrm{C}$ for the two and three pyrene backbone-containing GQs, respectively. Concurrently, CD spectra showed the formation of scaffolds with elevated content of parallel strands by the increase of the $260 \mathrm{~nm}$ peak. In the deep eutectic solvent of $1: 2$ choline chloride-urea containing $100 \mathrm{mM} \mathrm{KCl}$, mimicking viscous biological conditions, the $T_{m}$ values decreased from $68.8^{\circ} \mathrm{C}$ to $60.9,40.8$, and $36.6^{\circ} \mathrm{C}$ for the one, two, and three pyrene backbone-containing GQs, respectively. CD spectra of the unmodified GQ showed parallel folding, to which the pyrenemodified GQs converted from hybrid form with increasing pyrene content [221].

\section{Synthetic Nucleotides That Only Destabilized a GQ Structure}

\subsection{Destabilizing Base Derivatives}

6-Thioguanine $\left(s^{6} G\right)$ and 6-Mercaptopurine $\left(s^{6} P u\right)$. The medication 6-thioguanine (Figure 13, 1), used against leukemia and ulcerative colitis, has also been introduced into various GQ-forming sequences, such as the $\mathrm{TG}_{4} \mathrm{~T}, \mathrm{TG}_{5} \mathrm{~T}$, $\mathrm{TBA}$, and the htel repeats. In $\mathrm{K}^{+}$the $s^{6} \mathrm{G}$ in none of the $\mathrm{G}$ positions altered the characteristic $\mathrm{CD}$ spectra of $\left[\mathrm{TG}_{4} \mathrm{~T}\right]_{4}$ and $\left[\mathrm{TG}_{5} \mathrm{~T}\right]_{4}$ but sharply destabilized the structures, as Gros et al. reported [222]. $\mathrm{s}^{6} \mathrm{G}$ was also incorporated into position 13 of the two-tetrad-forming (htel-21)T. The GQ contained a br $^{8} \mathrm{G}$ at position 7 , as well, and the effect of $s^{6} \mathrm{G}$ on stability was not separately discussed. The overall topology was not changed by $s^{6} G$ [77]. However, $s^{6} G$ inhibited the formation of TBA's antiparallel, two-tetrad, chair-type GQ when it substituted for $G 2$ in $\mathrm{Gs}^{6} \mathrm{GT}_{2} \mathrm{G}_{2} \mathrm{TGTG}_{2} \mathrm{~T}_{2} \mathrm{G}_{2}$ or in $G_{2}(T U)_{2} G_{2}(U T)_{2} G_{2} U_{2} T_{2} G_{2}$ [223] although the $U$ base alone is not an inhibitor of GQ formation, for example, in $\mathrm{G}_{4}\left(\mathrm{~T}_{4} \mathrm{G}_{4}\right)_{3}$ [224], which is only one of the many cases known $[5,8]$. The destabilizing effect of $s^{6} \mathrm{G}$ was also studied by molecular dynamics calculations [225]. Destabilizing effect of a new purine analog, 6-mercaptopurine, has been described by Radhika et al. [226] based on MD simulations of the GQ structure of (TGGGGT) ${ }_{4}$ containing a single analog that also caused local distortion of the fold.

6-Selenoguanine ( $\left.\mathrm{se}^{6} \mathrm{G}\right)$. Molecular dynamics simulations of the TBA GQ showed that although one nucleotide of $\mathrm{se}^{6} \mathrm{G}$ was tolerated in the scaffold, the thermodynamic stability was reduced accompanied by conformational alterations. Two or more such mutations prompted unfolding of the scaffold due to steric clashes in the interior channel of the GQ, which led to the release of the central $\mathrm{K}^{+}$ion and to the disruption of the structure [227].

7-Deazaguanine $\left(c^{7} G\right)$. In GQ models, the 7-deazaguanine (Figure 13, 2) was first mentioned in 1992 demonstrating that "regular" GQ structures cannot be formed if $c^{7} G$ replaced a $G$ in the core [228]. Biological functions were also impaired by this analog, as it was shown when it substituted individually for each G-position of the 15-mer TBA DNA $[19,20]$. The TBA aptamer binds to and inhibits thrombin. 7-Deazaguanosine in each of the eight tetrad positions significantly reduced the inhibitory activity of TBA. Substitution of G8 of the central TGT loop had a minor effect as demonstrated by the 2 -fold increase in the inhibition constant. Later, $\mathrm{c}^{7} \mathrm{G}$ was also incorporated into the DNA sequences forming tetramolecular architectures, such as the $\mathrm{TG}_{4} \mathrm{~T}$ and $\mathrm{TG}_{5} \mathrm{~T}$ [222]. The modified oligonucleotides formed the same type of parallel structures as the unmodified ones did although the Hoogsteen-type H-bonding could not be formed in the absence of the H-acceptor of N7. Due to the steric requirements of the hydrogen atom of $\mathrm{C} 7$, the $\mathrm{c}^{7} \mathrm{G}$ tetrads became deformed as compared to the full-G tetrads, resulting in changes of stacking. This led to large reduction in the thermal stability of $\left[\mathrm{TG}_{5} \mathrm{~T}\right]_{4}$ by the $\mathrm{c}^{7} \mathrm{G}$-analogs. In general, intramolecular GQs cannot form or are severely destabilized by a single $c^{7} \mathrm{G}$ in the core, which made $c^{7} \mathrm{G}$ a "principle-proving" analog [229-232]. The $c^{7} G$ derivative 8-aza-7-deaza-isoguanine $\left(n^{8} c^{7} i G\right)$ (Figure 13,3$)$ nucleotidecontaining DNAs can form tetrads and also pentads and thus GQ and pentaplex scaffolds. Seela and Kröschel [39] described that the $\mathrm{T}_{4}\left(\underline{\mathbf{n}^{8} \mathbf{c}^{7} \mathbf{i G}}\right)_{4} \mathrm{~T}_{2}$ molecules self-assembled into GQs in the presence of $\mathrm{Na}^{+}$or $\mathrm{Rb}^{+}$, whereas they formed pentaplexes with $\mathrm{Cs}^{+}$cations. Structural stability of the GQ assemblies is not known. 


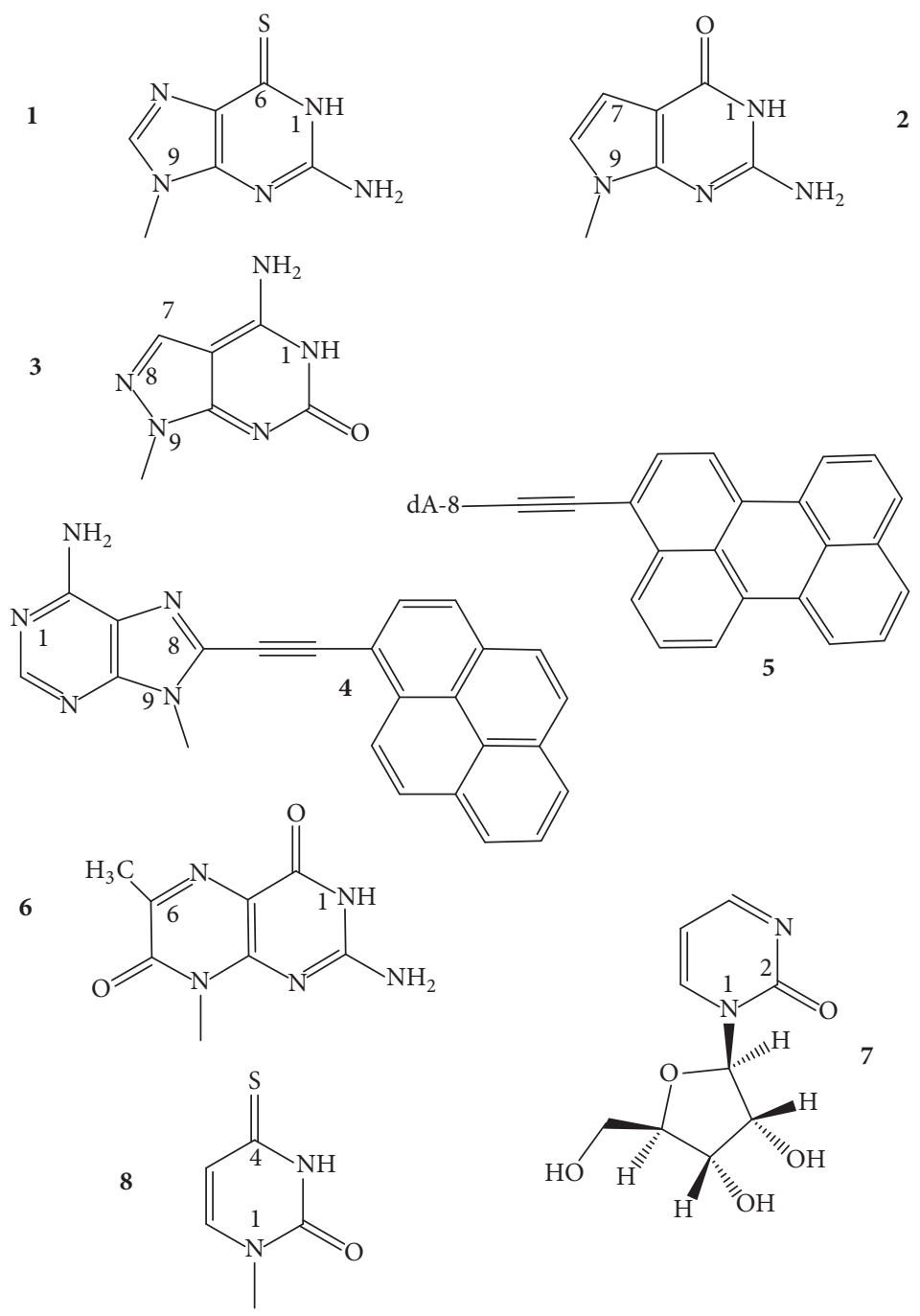

FIGURE 13: Structures of 6-thioguanine (1); 7-deazaguanine (2); 8-aza-7-deaza-isoguanine (3); pyrene (4) and perylene (5) tethered to C8 of guanine; 6-methyl-isoxanthopterin (6); zebularine or 2-pyrimidinone-riboside (7); and 4-thiouracil (8).

Pyrene-Perylene dA. GQ-induced FRET (fluorescence resonance energy transfer) has been studied by substituting loop adenosines at position 8 with a tethered FRET pair in A(htel21), A $(\mathrm{G})_{n} \operatorname{TTPyA}(\mathrm{G})_{n} \operatorname{TTPerA}(\mathrm{G})_{n} \operatorname{TTA}(\mathrm{G})_{n}$, where the $n$ changed from 2 to 4 , Py is pyrene (donor), and Per is perylene (acceptor) group (Figure 13, 4, 5). The substituted bases did not hinder the formation of intramolecular 2-, 3-, and 4tetrad GQs in $\mathrm{K}^{+}$; however, they marginally destabilized them by $1-2^{\circ} \mathrm{C}$, relative to the unmodified control GQs [233].

6-Methyl-isoxanthopterin. A fluorescent $\mathrm{G}$ analog 6-methylisoxanthopterin (6MI, Figure 13, 6) has been incorporated into the central TGT loop (position G10) and also at the middle G-tetrad (position G13) of the GQ-forming $\mathrm{G}_{3} \mathrm{~T}_{2} \mathrm{G}_{3}$ T ${ }_{\text {TTG }} \mathrm{GGT}_{2} \mathrm{G}_{3}$ [234], whose DNA is part of the promoter region of c-MYC oncogene. The loop modification slightly decreased the thermal stability of wild-type GQ, while the tetrad modification significantly did the same. CD spectrum of the wild-type and the modified ones displayed a strong positive band at $263 \mathrm{~nm}$ and a minor peak at $298 \mathrm{~nm}$, which the authors, probably mistakenly, called antiparallel basket and/or chair topologies. The fluorescence intensity of the loop-modified GQ was greater than the tetrad substituted, as the authors expected from the extent of stacking interaction, which is larger in the $\mathrm{G}(6 \mathrm{MI}) \mathrm{G}$ sequence than in the $\mathrm{T}(6 \mathrm{MI}) \mathrm{T}$ sequence. The fluorescence emission intensity decreased when $6 \mathrm{MI}$ was incorporated into single-stranded oligonucleotide and decreased further in the double-stranded oligonucleotide, demonstrating that its fluorescence emission intensity can be used to probe the microenvironment of $6 \mathrm{MI}$. The 6-methyl-isoxanthopterin was also substituted for the T4 (loop) of $\left(\mathrm{G}_{3} \mathrm{~T}\right)_{3} \mathrm{G}_{3}$ oligonucleotide to develop fluorescent detection methods. The wild-type sequence folded into parallel GQ in $\mathrm{K}^{+}$. The substitution did not change the original topology but destabilized the GQs. $T_{m}$ of the $\left(\mathrm{G}_{3} \mathrm{~T}\right)_{3} \mathrm{G}_{3} \mathrm{GQ \text {, }}$ measured in $10 \mathrm{mM} \mathrm{KCl}$, was $90^{\circ} \mathrm{C}$, which was reduced by $12^{\circ} \mathrm{C}$ by the base analog [124]. 
<smiles>CC(OP(C)(=O)[O-])C(CO)NC(=O)CBr</smiles><smiles>COCC(COC)(Cc1ccccc1)Cc1ccccc1</smiles>

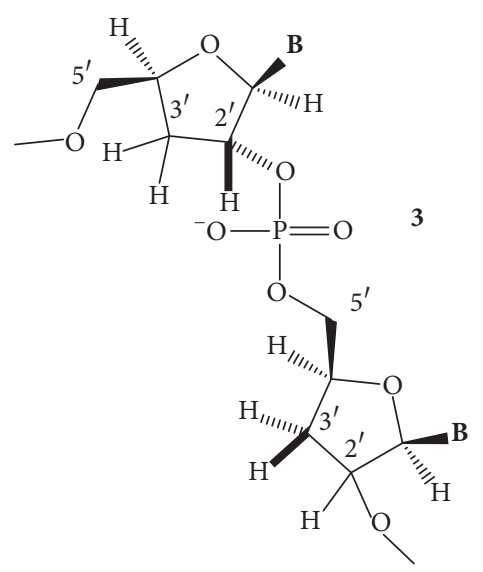

FIGURE 14: The acyclic threoninol (aTNA) nucleoside (1); the dibenzyl linker (2); and a $3^{\prime}$-deoxy-2 ${ }^{\prime}-5^{\prime}$-iso-dinucleotide (3).

2-Pyrimidinone and 4-Thiouracil Nucleosides. Mammalian telomeric DNA is transcribed into RNA that contains the 6-mer uuaggg repeats [235]. Sequence analogs of this, like the uagggu, and those containing analogs of uridines, the zebularine (ribonucleoside of 2-pyrimidinone) that does not have the $\mathrm{O} 4$ atom, and the 4 -thiouridine $\left(s^{4} \mathrm{U}\right)$ (Figure $13,7,8$ ), in which the sulfur atom cannot develop $\mathrm{H}$ bond, have been prepared. The tetramolecular parallel GQs formed by the wild types have been radically destabilized by $s^{4} \mathrm{U}: T_{m}$ value of $79^{\circ} \mathrm{C}$ for [uagggu] $]_{4}$ was reduced in [uagggs $\left.{ }^{4} \mathrm{U}\right]_{4}$ to $49.4^{\circ} \mathrm{C}$ [236]. Mendelboum and coworkers [237] prepared 4-thiouracil- $2^{\prime}$-deoxyuridine- ( $\left.\mathrm{s}^{4} \mathrm{dU}-\right)$ containing TBA oligonucleotides, in which the base analog substituted for thymines of the loop sequences. Replacement of four thymines by $\mathrm{s}^{4} \mathrm{dU}$ in the 15 -mer resulted

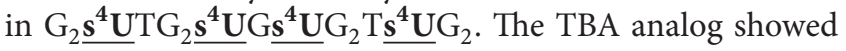
increased anticoagulant and antithrombotic properties relative to the unmodified TBA. The increased activity was explained by the altered properties of $s^{4} \mathrm{dU}$ as compared to thymine. The substitutions rendered the molecule more hydrophobic, which might have been preferable for the aptamer-thrombin interaction; furthermore, the $s^{4} \mathrm{dU}$ containing oligonucleotides have been described as highly resistant to cellular nucleases. This aptamer analog contained more than $26 \%$ of thiolated nucleotides, and thus it must have been more stable in a biological environment than its unmodified counterparts. Thermodynamic stability of the $s^{4} \mathrm{U}-\mathrm{TBA}$ GQ was not specified [237].

\subsection{Destabilizing Substitutions of the Sugar and Backbone Moieties}

Acyclic Threoninol. An acyclic sugar analog, the acyclic threoninol (aTNA)-guanine (Figure 14, 1), has been substituted by Zhou's group [238] for each guanine, one by one in $\mathrm{TG}_{4} \mathrm{~T}$. Based on the $\mathrm{CD}$ spectra, all the modified oligonucleotides could form GQ structures and only the G3-substituted TGGGGT's conformation differed from the tetramolecular parallel GQ structures formed by the natural counterpart, $\left[\mathrm{TG}_{4} \mathrm{~T}\right]_{4}$. This modified oligonucleotide built multiple scaffolds. Thermal stability of $\left[\mathrm{TG}_{4} \mathrm{~T}\right]_{4}$ was reduced by the aTNA-G in each case, and the modification at the $5^{\prime}$ and $3^{\prime}$-terminal G-tetrads was the most detrimental to the stability [238].

Dibenzyl Linker. TBA is primarily known as an anticoagulant aptamer. Another less known biological activity of TBA is its anticancer potential, which is rather hindered by the anticoagulant action, as reported by Scuotto et al. [239]. They found that replacing one residue of the TT or TGT loops with a dibenzyl linker (Figure 14, 2), with which seven new GQforming TBA sequences were created, could maintain the antiproliferative activity over the anticoagulant activity. Most T-substitutions only slightly affected the thermal stability of the wild-type TBA, except the T9-modification that reduced $T_{m}$ of TBA $\left(50.7^{\circ} \mathrm{C}\right.$ in $\left.90 \mathrm{mM} \mathrm{KCl}\right)$ by $15^{\circ} \mathrm{C}$. The T13-modified analog possessed selective antiproliferative activity, while the T12 analog retained the potent anticoagulant activity of 
the unmodified TBA. Structural analyses indicated that the different localization of the two benzene rings of the linker was responsible for the loss of the antithrombin activity of the $\mathrm{T} 13$ analog.

Methylphosphonate. In the methylphosphonate (P-Me, Figure 12,3 ) internucleotide linkages, a methyl group replaces one of the nonbonded oxygen atoms, and thus the internucleotide linkage becomes uncharged, neutral. This causes the ion-solvating water spine-perturbed along the backbone, resulting in the destabilization of a folded structure. With P-Me linkages-containing $\left[\mathrm{TG}_{4} \mathrm{~T}\right]_{4},\left[\mathrm{G}_{4} \mathrm{~T}_{4} \mathrm{G}_{4}\right]_{2}$, and the TBA GQs, no thermal transition was detected, and the $\mathrm{AG}_{3}\left(\mathrm{TTAG}_{3}\right)_{3} \mathrm{GQ}$ was powerfully destabilized [1].

isoDNA. The name refers to the modification of the internucleotide phosphodiester linkage, in which the natural phosphodiester bond, $3^{\prime} \mathrm{O}-\mathrm{OP}\left(\mathrm{O}^{-}\right)-5^{\prime} \mathrm{O}$, of nucleic acids is changed, for instance, to $2^{\prime} \mathrm{O}-\mathrm{OP}\left(\mathrm{O}^{-}\right)-5^{\prime} \mathrm{O}$, which is the $2^{\prime}-5^{\prime}$ isoDNA (Figure 14, 3). The fully $2^{\prime}-5^{\prime}$ linked isoTBAs formed unimolecular antiparallel GQs in the presence of $\mathrm{K}^{+}$ions, similar to how the unmodified, $3^{\prime}-5^{\prime}$-linked TBA oligonucleotides did. The isomeric TBA had lower thermal stability than the native one. The TBA's $T_{m}$ value in $\mathrm{K}^{+}$was $52^{\circ} \mathrm{C}$, while the isoTBA GQ's was $37.1^{\circ} \mathrm{C}$. When the T7 and $\mathrm{T} 9$ were replaced by $\mathrm{U}$ nucleotides, the stability increased up to $45^{\circ} \mathrm{C}$. On the other hand, the isoTBAs exhibited higher stability against exonucleases and were capable of retaining the biological function of the native TBA, that is, slowing down the process of blood clotting [240]. The antiparallel chair-type TBA GQ, whose nucleotides are connected via 3' $5^{\prime}$ phosphodiester linkages, folded into parallel GQ when the 2-3-2-nucleotide-long loops (TT...TGT...TT) were shortened. The $2^{\prime}-5^{\prime}$-isoTBA analog, interestingly, retained the antiparallel topology with the shorter loops such as 2-2-2 or 13-1 or even 1-1-1 nucleotides of the resulting 14-, 13-, or 11-mer GQs. Thermal stabilities of the GQs were, however, reduced by the introduction of shorter loops into the $2^{\prime}-5^{\prime}$-isoTBA sequences, from $T_{m}$ of $48^{\circ} \mathrm{C}$ for the unmodified $3^{\prime}-5^{\prime}$ TBA to $34^{\circ} \mathrm{C}$ for the isoTBA $(232)$ and down to $20^{\circ} \mathrm{C}$ for the isoTBA(111) in $100 \mathrm{mM} \mathrm{KCl} \mathrm{[241].}$

\section{Natural Base Lesions and Epigenetic Modifications in GQ DNAs}

Exogenous and endogenous chemicals and radiation cause many types of lesions in cellular DNA. The nucleotides are modified by different types of reactions, such as oxidation, alkylation, and hydrolysis. These alterations are widespread and play an important role in changing the physiological states of cells and can thus lead to various diseases. Majority of genetic impairments are believed to originate from oxidative processes, which are the basis of mutation, aging, cell death, and carcinogenesis [242-244]. Reactive oxygen and nitrogen species (ROS and RNS) arising endogenously and originating mainly from the cell's aerobic metabolism also contribute to the age-dependent diseases [244-246]. There are $\sim 80$ known DNA defects that can form upon the initial attack of ROS and RNS [247]. Among the exogenous effects, the damage can arise from radiation, directly from the ionizing energy or indirectly from hydroxyl radicals that form by the ionization of the solvation shell around the DNA [248, 249]. The radical cation (electron ionization hole) can travel hundreds of Angstroms by hopping before being entrapped, preferentially by purine bases $[250,251]$. Oxidation of purines initially leads to 8-oxo-7,8-dihydroguanosine $\left(\mathrm{o}^{8} \mathrm{G}\right), 8$-oxo7,8-dihydroadenosine $\left(\mathrm{o}^{8} \mathrm{~A}\right)$, and 8-oxo-7,8-dihydroinosine $\left(0^{8} \mathrm{I}\right)$ in RNA, in DNA, and in the nucleotide pool [244]. The effects of natural base lesions on the structure and stability of double-stranded DNA models have been extensively studied (see [252-258] and the references therein). Majority of the lesions are promutagenic and procarcinogenic if not repaired in due time. The base excision repair (BER) pathway, regulated by many different types of enzymes including DNA glycosylases, abasic endonucleases, phosphodiesterases, DNA polymerases, and DNA ligases, is responsible for the accurate removal of the lesion and the restoring of the original state of the double-stranded nucleic acids [243]. Due to imperfections of the repair system, the lesions that are not repaired can severely damage the DNA structure where they are formed. Base damage also occurs in noncanonical DNA structures such as the GQs. Studying the lesion-damaged GQ structures using various GQ models is less than a decade old. A few cases are known only about repairing damaged GQs, such as the N6-methylguanine $\left(\mathrm{m}^{6} \mathrm{G}\right)[259]$ and the further oxidized derivatives of 8 -oxoguanine $\left(\mathrm{o}^{8} \mathrm{G}\right)$, but $\mathrm{o}^{8} \mathrm{G}$ is not among them [260].

Using GQ models, the $\mathrm{o}^{8} \mathrm{G}, \mathrm{o}^{8} \mathrm{~A}, \mathrm{n}^{8} \mathrm{~A}$ (8-aminoadenine), $\mathrm{m}^{6} \mathrm{G}, \mathrm{G}$ - and A-abasic (AP) sites, $\mathrm{hm}^{5} \mathrm{U}$, hypoxanthine (I), xanthine $(\mathrm{X})$, and the cyclobutane thymine dimers have been investigated for the effect on GQ stability. Most natural DNA damage is destabilizing both in canonical and in noncanonical nucleic acids; a few, however, do not affect or even stabilize the GQ fold. The stabilizing lesions are the ${ }^{8} \mathrm{~A}$ and $\mathrm{n}^{8} \mathrm{~A}$, only if located in the loops of the htel GQs, and also $0^{8} \mathrm{G}$ if located in the tetramolecular DNA GQs of $\left[\mathrm{TG}_{4} \mathrm{~T}\right]_{4}$ and $\left[\mathrm{TG}_{5} \mathrm{~T}\right]_{4} \cdot \mathrm{hm}^{5} \mathrm{U}$, with its minor effects, also belongs to this group of analogs as well as the AP site when replacing a single-base loop in GQs of the $T\left(G_{3} T\right)_{4}$ and $\left(G_{2} \underline{A P}\right)_{3} G_{2}$.

\subsection{Natural Base Lesions That Stabilize or Destabilize, Depending on the GQ Structure}

8-Oxo-2 $2^{\prime}$-deoxyguanine $\left(o^{8} d G\right)$. Guanine has the lowest redox potential among the four DNA bases [251]; therefore, guanine is the major site of oxidation in DNA by ROS, the reactive oxygen species [261]. Among the numerous oxidative derivatives, the 7,8-dihydro-8-oxoguanine (8-oxoguanine, $0^{8} \mathrm{G}$, Figure 15,1$)$ was found to be the major primary product in vivo [262-264]. Since in vivo levels of $0^{8} \mathrm{G}$ were found to be safely measurable, being between 0.3 and $40^{8} \mathrm{G}$ per $10^{6} \mathrm{G}$, $\mathrm{o}^{8} \mathrm{G}$ has been used as a marker of oxidative stress of cells $[265,266] .0^{8} \mathrm{G}$ is even more prone to oxidation than $\mathrm{G}$ as its redox potential is even lower [267]. Further oxidation of $o^{8} \mathrm{G}$ results in guanidinohydantoin and spiroiminodihydantoin, among other minor products $[268,269]$. Consecutive runs 

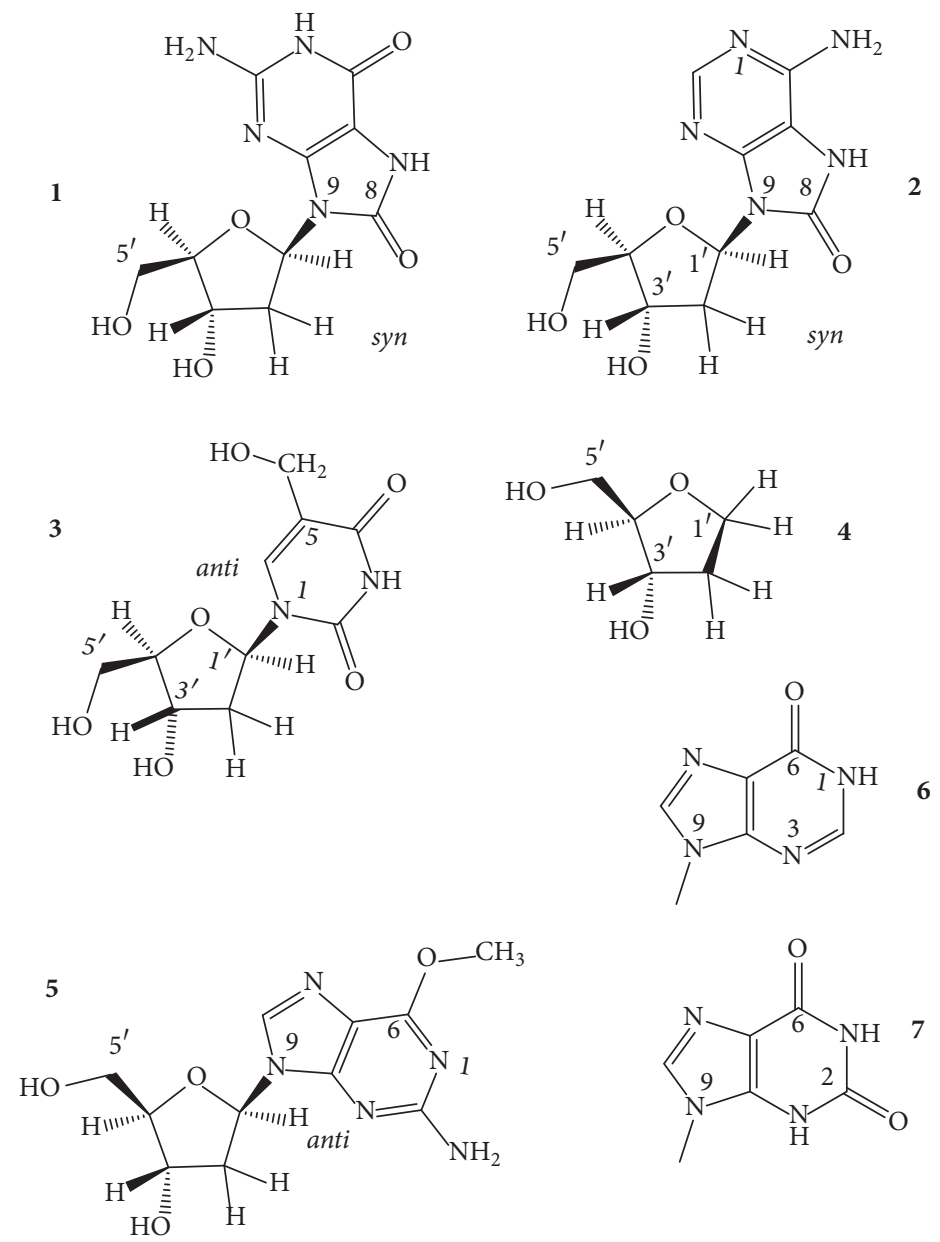

FIGURE 15: Structures of the syn 8-oxo-2' -deoxyguanosine (1); syn 8-oxo-2' -deoxyadenosine (2); anti 5-hydroxymethyl-2' -deoxyuridine (3); the tetrahydrofuranyl abasic site (4); anti O6-methyl-2' -deoxyguanosine (5); hypoxanthine (6); and xanthine (7).

of guanines, as those in the potential GQ-forming G-rich sequences, further lower the redox potential of guanine since these runs act as sinks for the oxidative damage [270]. The glycosylases known to repair $0^{8} \mathrm{G}$ in duplex DNA, such as OGG1, NEIL1, and NEIL3, cannot remove ${ }^{8} \mathrm{G}$ from DNA GQs, although these enzymes do repair guanidinohydantoin and spiroiminodihydantoin in GQs [260]. Therefore, the unrepaired, persistent $0^{8} \mathrm{Gs}$ can ruin the GQ structure. This has been suggested to lead to telomere shortening and finally to cellular senescence [271]. The effect of $o^{8} \mathrm{G}$ was, however, recently proved by Fouquerel and coworkers to be not unambiguous, as the destabilized, partially unfolded GQ could promote telomerase activity that could lead to telomere extension $[272,273]$. Formation of $o^{8} \mathrm{G}$ in GQs motivated wide-ranging investigations.

The 8-oxoguanine was first studied with tetramolecular parallel GQ structures by Gros et al. [222] using the $\left[\mathrm{TG}_{4} \mathrm{~T}\right]_{4}$ and $\left[\mathrm{TG}_{5} \mathrm{~T}\right]_{4}$ models. These GQs assemble from four $\mathrm{TG}_{4} \mathrm{~T}$ and $\mathrm{TG}_{5} \mathrm{~T}$ strands, respectively, and therefore the presence of a single modified base in one strand forms a modified tetrad in the GQ. Incorporation of a single $0^{8} \mathrm{dG}$ into each G-position of $\mathrm{TG}_{4} \mathrm{~T}$ and $\mathrm{TG}_{5} \mathrm{~T}$ did not hinder the formation of tetramolecular parallel GQs. Interestingly, $\mathrm{o}^{8} \mathrm{dG}$ proved to be a stabilizing modification, especially when incorporated at the $5^{\prime}$ position of the G4 or G5 sequence, as characterized by the $T_{1 / 2}$ values. As the preferred glycosidic conformation of $o^{8} \mathrm{dG}$ is $\operatorname{syn}$ (Figure 15, 1), similar to the majority of 8modifications of $G$ [63], and in most cases in the parallel GQs all nucleosides have anti glycosidic torsion angle, $o^{8} \mathrm{G}$ was supposed to destabilize the structure. Furthermore, in the 6,8-diketo tautomeric form of o ${ }^{8} \mathrm{G}$ [274], the donor-acceptor arrangement of the Hoogsteen $\mathrm{H}$-bonding changes, which again would have predicted the destabilization of the parallel GQ by the $0^{8} \mathrm{dG}$ tetrad. The observed stabilization effect was finally explained by strong stacking interactions with the neighboring anti G-quartet. As it turned out later, the elevated stability was observed merely with these two tetramolecular scaffolds. There was another experiment for tetrad formation from $o^{8} \mathrm{G}$ nucleotides when $0^{8} \mathrm{dG}$ replaced $\mathrm{G}$ in the four $5^{\prime}$ positions of the GGG triplets (in bold, underlined) in GGGTGGGTGGGTGGG and results were the opposite, destabilization. This oligodeoxynucleotide folded into an intramolecular parallel GQ, possibly due to the single-base loops. Thermostability of wild type was substantially reduced 
by o ${ }^{8} \mathrm{dG}$, by $10^{\circ} \mathrm{C}$ in $\mathrm{Na}^{+}$, and no unambiguous $T_{m}$ value could be determined in $\mathrm{K}^{+}$where an even larger destabilization was apparent. The circular double $\mathrm{H}$-bonded scheme was assumed to consist of N1H-O8G and O6G-HN7 [275].

Reduced stability and conformational changes were observed when a single $0^{8} \mathrm{dG}$ was incorporated into the GQ-forming htel oligonucleotides. Szalai et al. [276] used the 25-mer model A(htel-21)TGT and found that $\mathrm{o}^{8} \mathrm{G}$ at $5^{\prime}$ positions of the GGG triplets retained the intramolecular antiparallel topology of the wild type, whereas $0^{8} \mathrm{G}$ in the middle positions of the triplet caused formation of multiple folds. With the 24-mer hybrid GQ of TT(GGGTTA) ${ }_{3}$ GGGA, Lech et al. [80] detected significant destabilization of the wild type even in cases when the syn dGs were changed to $\mathrm{o}^{8} \mathrm{dGs}$ in positions 3,9 , and 15 (all $5^{\prime}$ positions in the GGG triplets) and the $\Delta T_{m}$ values ranged between -9.8 and $-18.5^{\circ} \mathrm{C}$. Concurrently, a mixture of topologies was formed. $\mathrm{o}^{8} \mathrm{dG}$ was also incorporated into all $12 \mathrm{dG}$ positions (syn and anti) one by one, into the GQ-forming htel-21, $\mathrm{G}_{3}\left(\mathrm{TTAG}_{3}\right)_{3}$ by Sagi and coworkers [26]. The study revealed that the GQ structures were substantially destabilized by enthalpydriven effects both in $\mathrm{Na}^{+}$and in $\mathrm{K}^{+}$solutions. The negative effect was position-dependent and varied with the cation used. When the single ${ }^{8} \mathrm{dG}$ was located in the two terminal tetrads, the $\mathrm{Na}^{+}$-stabilized basket-type antiparallel topology was also retained in $\mathrm{K}^{+}$, contrary to the wild-type htel-21, which forms a mixture of folds, hybrids, and $\mathrm{K}^{+}$-antiparallels in $\mathrm{K}^{+}[68,101,277,278]$. The middle tetrad substitutions by $0^{8} \mathrm{dG}$ caused the largest reductions in stability ( -19 to $-26^{\circ} \mathrm{C}$ in $\mathrm{Na}^{+}$and -27 to $-30^{\circ} \mathrm{C}$ in $\mathrm{K}^{+}$), as observed before with other base mutations $[279,280]$, and various folds were observed too. The damaging effect of $\mathrm{o}^{8} \mathrm{dG}$ on the stability of monomolecular htel GQs was surprising in light of its stabilizing effects with tetramolecular GQs [222] (the o ${ }^{8} \mathrm{dG}$ triggered only marginal destabilizations in deoxyoligonucleotide duplexes and the effect was also length-dependent: with duplexes of 15 nucleotides long, even a small stabilization was observed; see references in [26]). In an $\mathrm{o}^{8} \mathrm{G}-\mathrm{G}-\mathrm{G}-\mathrm{G}$ tetrad, the 8-substitution changes the Hoogsteen-type circular H-bonding pattern. In the 6,8-diketo tautomeric form of $\mathrm{o}^{8} \mathrm{G}$ [274], the N7 is a hydrogen donor, instead of acceptor, and therefore only a single $\mathrm{H}$-bonding pattern remains when $\mathrm{o}^{8} \mathrm{G}$ is present in the tetrad. The weakened H-bonding and the stacking changes induced by the altered tetrad can result in the extensive destabilization of the GQ.

As $\mathrm{o}^{8} \mathrm{dG}$ contained in htel GQ was found not to be a substrate of the glycosylase enzymes that repair $0^{8} \mathrm{dG}$ in duplex DNA, such as the hOOG1 and NEIL enzymes [260], $\mathrm{o}^{8} \mathrm{G}$ can become a persistent lesion in GQs. Surprisingly, this persistence was without any immediate negative consequences on the stability of telomere complexes in vivo (see references in [281]). An and coworkers have built a 31-mer telomeric GQ model from TAGGG(TTAGGG) $)_{4}$ TT, which contained five G-triplets, instead of the usual four, in which the dGs at the $5^{\prime}$-positions of 3,15 , and 27 were replaced by ${ }^{8} \mathrm{dG}$. These modified oligodeoxynucleotides folded into hybrid-1 or hybrid-2 GQ form. They observed that the folded structures could effectively accommodate a single ${ }^{8} \mathrm{dG}$ by looping out the damaged G-tract and allowing the other four G-triplets to adopt the hybrid fold. This change caused only a minimal negative impact on the stability of the GQ [281], contrary to the results obtained with the four G-tract telomeric GQ models in vitro. Considering the availability of additional G-tracts in the telomeric complexes, the authors explained how the persistent presence of a lesion can exist without immediate destabilization effect on the DNA-protein complex in vivo.

Another way was also found to offset the intense destabilization caused by a single $0^{8} \mathrm{dG}$ of the GQs built from the four TTAGGG repeat htel sequences and this was by the proper site-specific incorporation of another modified base, specifically xanthine (2,6-dioxopurine, X, Figure 15, 7). Xanthine is another natural derivative of guanine (see in the section Xanthine). X can also be assumed to be a destabilizing analog due to the loss of $\mathrm{H}$-bonds if incorporated into a $\mathrm{G}$ tetrad, similarly, as it was found with hypoxanthine (I for inosine) (see under section Hypoxanthine). The outcome of the $o^{8} \mathrm{dG}-\mathrm{X}$ double modification depends on the positions of the modified nucleotides. Benz and Hartig [282] incorporated first two ${ }^{8} \mathrm{G}$ and two $\mathrm{X}$ nucleotides into the TA(htel21) $\mathrm{T}$ sequences, which folded into $\mathrm{X}: 0^{8} \mathrm{G}: \mathrm{X}: 0^{8} \mathrm{G}$ tetradscontaining GQs. The modified tetrads destabilized the wildtype GQ and the topology depended on the arrangement. The GQ either remained in the basket-type antiparallel or changed into parallel form:

(i) wild-type: TA GGG TTAGGG TTAGGG TTAGGG T antiparallel

(ii) $\mathrm{o}^{8} \mathrm{G}(3): \mathrm{X}(11): \mathrm{X}(15): \mathrm{o}^{8} \mathrm{G}(23)$ : TA $\underline{\mathrm{O} G G}$ TTAGG TTAX $\underline{X} G$ TTAGG $\underline{\mathrm{O}} \mathrm{T}$ antiparallel

\section{(iii) $\mathrm{o}^{8} \mathrm{G}(3): \mathrm{X}(9): \mathrm{o}^{8} \mathrm{G}(15): \mathrm{X}(21)$ : TA $\underline{\mathrm{O} G G}$ TTAXGG TTAㅁG TTAX́GG T parallel}

Later, Cheong et al. [283] found the positions for $\mathrm{o}^{8} \mathrm{G}$ and $\mathrm{X}$ that could compensate for the negative effect of $\mathrm{o}^{8} \mathrm{G}$ on the stability of the wild type and which also retained the original topology. They inserted the G:G:X:0 ${ }^{8} \mathrm{G}$ tetrads into a series of TT(htel-21)A deoxyoligonucleotides. Conformation of the modified GQ scaffolds remained similar to the original hybrid-1 fold. Three of the modified GQs were destabilized, by $3^{\circ} \mathrm{C}$ to $12^{\circ} \mathrm{C}$ in their $T_{m}$; two had similar stability as the wild type had $\left(56^{\circ} \mathrm{C}\right)$, and the $\mathrm{G}(3): 0^{8} \mathrm{G}(9): \mathrm{X}(17): \mathrm{G}(21)$ tetrad proved to be stabilizing, by $2^{\circ} \mathrm{C}$ in its $T_{m}$ relative to the wild type. Cheong and coworkers [284] could also reverse the polarity of $\mathrm{H}$-bonds in a tetrad while the original folding topology of the wild type was preserved.

The effect of $\mathrm{o}^{8} \mathrm{dG}$ has been well demonstrated to depend on the secondary structure of nucleic acids [26, $285,286]$. Recent studies provided additional results, such as the comparison of the effect of $\mathrm{o}^{8} \mathrm{dG}$ in triplexes and GQs [100], the involvement of $0^{8} \mathrm{dG}$ in the stabilization of "guanine-vacancy-bearing" GQs [287], or a comprehensive study on the complex effect of metal ions and cosolutes on the topology of the A(htel-21) GQ when both $0^{8} \mathrm{G}$ and xanthine were contained in the tetrads [288]. 8-Oxoguanine in GQ loops accommodated well and did not have much effect on 
the stability [281]. The Zn(II)-porphyrin complex-induced oxidation of guanines in the GQ of TA(htel-21) yielded $o^{8} \mathrm{G}$ and its further oxidized product, the spiroiminodihydantoin. The oxidized bases prompted structural rearrangements of the parallel and hybrid TA(htel-21) GQs into an antiparallellike conformation [289], such as what was observed earlier with $0^{8} \mathrm{G}$-containing htel-21 GQs [26].

8-Oxo-2 $2^{\prime}$-deoxyadenosine $\left(o^{8} d A\right)$. The first study with 7,8 dihydro-8-oxo- $2^{\prime}$-deoxyadenosine (8-oxodA or $\mathrm{o}^{8} \mathrm{dA}$, Figure 15, 2), another oxidative natural base lesion, was carried out by Esposito et al. [290] and Petraccone et al. [291] using the tetramolecular parallel GQ DNA models of [AGGGT] and $[\text { TAGGGT] }]_{4}$. All the modified oligonucleotides formed the same parallel-type tetramolecular GQs as the unmodified two oligonucleotides did. The $0^{8} \mathrm{dA}$ substituting for $\mathrm{dA}$ nucleotides in both structures decreased the thermostability of the parent GQs by $14^{\circ} \mathrm{C}$ and $8^{\circ} \mathrm{C}$, respectively, according to absorption-based $T_{m}$ measurement, and the drastic negative effects were supposed to originate from the formation of the $0^{8}$ dA-tetrads [290]. Calorimetry, however, provided different results: the $[\mathrm{AGGGT}]_{4}$ GQ was not destabilized by the analog, formulated as 8-hydroxy-dA, oh ${ }^{8} \mathrm{dA}$ [291].

Among the htel GQ models, $\mathrm{o}^{8} \mathrm{dA}$ was first incorporated into the A(htel-21) GQ by Aggrawal et al. [292]. The analog moderately stabilized, did not affect, or slightly destabilized the GQ depending on the position of substitution. Single, double, and triple substitutions by $0^{8} \mathrm{dA}$ were also investigated. In $\mathrm{Na}^{+}$the average $\Delta T_{m}$ of four single substitutions was $\sim 0.5^{\circ} \mathrm{C}$ above the wild-type's $T_{m}$ of $60.1^{\circ} \mathrm{C}, 1^{\circ} \mathrm{C}$ in $\Delta T_{m}$ of three double modifications, and $4.1^{\circ} \mathrm{C}$ of the triple. In $110 \mathrm{mM} \mathrm{K}^{+}$ solution the respective values were $2.5^{\circ} \mathrm{C}, 2.7^{\circ} \mathrm{C}$, and $8.9^{\circ} \mathrm{C}$. The presence of $\mathrm{o}^{8} \mathrm{~A}$ in the loops of $\mathrm{A}(\mathrm{htel}-21) \mathrm{GQ}$ did not change the intramolecular antiparallel conformation in $\mathrm{Na}^{+}$, but in $\mathrm{K}^{+}$multiple folds were shown by the $\mathrm{CD}$ spectra. The stabilization by $0^{8} \mathrm{~A}$ was explained by the tight binding of $\mathrm{K}^{+}$ into the pocket formed by the O8 of $0^{8} \mathrm{~A}$ and its loop [292]. In another study [293], the effect of $0^{8} \mathrm{~A}$ was compared with the effect of other natural loop lesions, such as the adenine abasic (A/AP) site and 5-hydroxymethyluracil $\left(\mathrm{hm}^{5} \mathrm{U}\right)$ in the GQ of A(htel-21). $\mathrm{o}^{8} \mathrm{~A}$ stabilized the $\mathrm{Na}^{+}$-basket and the $\mathrm{K}^{+}$stabilized folds by $1.7^{\circ} \mathrm{C}$ and $1.1^{\circ} \mathrm{C}$, respectively, as averages of the effects at the four sites. The $\mathrm{hm}^{5} \mathrm{U}$ (Figure 15, 3) hardly affected the stability, while the AP site (dSpacer; Figure 15, 4) destabilized the GQ structure [293].

5-Hydroxymethyluracil $\left(\mathrm{hm}^{5} \mathrm{U}\right)$. One of the main natural oxidation products by ROS of the DNA thymine is $\mathrm{hm}^{5} \mathrm{U}$ (Figure 15, 3). The ten eleven translocation (Tet) enzymes oxidize the epigenetically important base 5-methylcytosine stepwise to 5-hydroxymethylcytosine, 5-formylcytosine, and 5-carboxycytosine. Pfaffeneder et al. [294] found that Tet enzymes also oxidized $\mathrm{T}$ to $\mathrm{hm}^{5} \mathrm{U}$ in mouse embryonic stem cells. The $\mathrm{hm}^{5} \mathrm{U}$ also forms by ionizing radiation and oxidation of the deaminated $\mathrm{m}^{5} \mathrm{C}$ [295]. $\mathrm{hm}^{5} \mathrm{U}$ was first incorporated into the $5^{\prime}$-T of TGGGT oligonucleotide that readily formed the tetramolecular parallel GQ. $\mathrm{hm}^{5} \mathrm{U}$ was modeled as forming an $\mathrm{hm}^{5} \mathrm{U}$-tetrad via an extra $\mathrm{H}$-bond through the 5-OH group [296]. Virgilio et al. [297] replaced each thymine in the three loops of the TBA DNA sequences by $\mathrm{hm}^{5} \mathrm{U}$. All six sequence analogs retained the ability of the native TBA oligonucleotide to fold into the antiparallel, chair-like, two-tetrad GQ. $\mathrm{hm}^{5} \mathrm{U}$ did not affect or slightly, by $1-3^{\circ} \mathrm{C}$, increased the thermal stability of the native $\mathrm{GQ}$, except for the $\mathrm{hm}^{5} \mathrm{U}$ in position 9 of the central TGT loop, which increased $T_{m}$ of the native by $6^{\circ} \mathrm{C}$. All TBA analogs showed decreased affinities to thrombin [297]. Effect of $\mathrm{hm}^{5} \mathrm{U}$ on the stability and conformation of htel GQs was described in 2015 by two laboratories [293, 298]. Both groups found that the replacement of a T of a TTA loop by $\mathrm{hm}^{5} \mathrm{U}$ resulted only in negligible effects on stability and did not affect the intramolecular topology of the native GQ. Sagi and collaborators [293] replaced each of the six T nucleotides in $\mathrm{A}$ (htel-21), the AGGG(TTAGGG) ${ }_{3}$ and the $\Delta T_{m}$ values, determined by absorption-based thermal melting profiles ranged from $-1.2^{\circ} \mathrm{C}$ to $0.5^{\circ} \mathrm{C}$ in $\mathrm{Na}^{+}$and from $-0.5^{\circ} \mathrm{C}$ to $0.8^{\circ} \mathrm{C}$ in $\mathrm{K}^{+}$solutions, both containing $0.169 \mathrm{mM}$ of the cations. Virgilio et al. [298] observed only stabilization by $\mathrm{hm}^{5} \mathrm{U}$ when six thymines were substituted either in $\mathrm{A}(\mathrm{htel}-21)$ or in the 26-mer (TTAGGG) ${ }_{4}$ TT DNA GQs. With the former GQ, the $\Delta T_{m}$ values ranged between 0.5 and $2.4^{\circ} \mathrm{C}$ and with the latter between 0.2 and $2.4^{\circ} \mathrm{C} . T_{m}$ values were determined here by calorimetry in $100 \mathrm{mM} \mathrm{K}^{+}$solutions.

Abasic Site (AP Site). Purine and pyrimidine abasic sites (AP sites) are among the most frequent lesions in cellular nucleic acids formed via spontaneous base loss, mainly depurination, and as intermediates in the enzymatic base excision repair process of various other base lesions [299]. Thousands of purine bases are released from DNA in every human cell daily and the resulting AP sites are highly mutagenic if they are not repaired. Natural AP sites occur in equilibrium states between the hemiacetal and the aldehyde forms. Due to the aldehydes, the AP sites are unstable leading to DNA chain breaks through beta-elimination [299]. Therefore, mostly stabilized forms of AP sites, primarily the tetrahydrofuranyl analog (dSpacer, Figure 15, 4), have been used in studies of abasics with canonical, double-stranded oligodeoxynucleotide models (e.g., [252, 253]). With noncanonical models, the effect of abasic lesions was a rather unexplored area until up to a decade ago $[20,300,301]$. With GQs, the well-known tetramolecular model assembling from $\mathrm{TG}_{5} \mathrm{~T}$ was first studied in 2010 by Esposito et al. by replacing the dGs by dSpacer AP sites (G/AP) [302]. In the same year, a natural model, the GQ of a human telomeric repeat sequence $\mathrm{G}_{3}$ (TTAGGG) ${ }_{3}$, the htel-21 was investigated for the effect of loss of G in G-tetrads by Sagi and coworkers [303]. Later, the GQ of the A(htel-21) was used as a model by Fujimoto's group [304]. This study also examined the effect of the crowding environment on abasic scaffolds. In 2012, the TA(htel-21) GQ was the abasic model of Virgilio et al. [305]. The main and shared conclusions of these studies were that the presence of a single G/AP in the G-tetrads did not hinder the formation of the tetramolecular or the monomolecular scaffolds. On the other hand, a single G/AP significantly decreased the thermal and thermodynamic stability of the parent GQ in a positiondependent way. With the three-tetrad htel scaffolds, the 
substitutions in the middle tetrad led to the largest reduction of stability. For instance, in $\mathrm{K}^{+}$a single G/AP incorporated into the two outer G-tetrads of the three-tetrad GQ of htel-21 decreased $T_{m}$ of $73^{\circ} \mathrm{C}\left(\Delta G^{\circ}{ }_{37}-6.44 \mathrm{kcal} / \mathrm{mol}\right)$ of the wild type by $10-17^{\circ} \mathrm{C}$ and by $1.76-3.43 \mathrm{kcal} / \mathrm{mol}$ in free energy changes $\left(\Delta \Delta G^{\circ}{ }_{37}\right)$ and by $21-26^{\circ} \mathrm{C}$ in the middle tetrad. (Due to the formations of multiple folds in the middle tetrad-substituted sequences, no thermodynamic data were calculated from the absorption-temperature profiles.) In $\mathrm{Na}^{+}$the wild-type GQ $\left(T_{m} 68^{\circ} \mathrm{C}, \Delta G^{\circ}{ }_{37}-4.75 \mathrm{kcal} / \mathrm{mol}\right)$ was destabilized by $3-17^{\circ} \mathrm{C}$ and $0.56-2.92 \mathrm{kcal} / \mathrm{mol}$ in the outer tetrads and by $12-23^{\circ} \mathrm{C}$ and $1.99-3.8 \mathrm{kcal} / \mathrm{mol}$ in the middle tetrad [303].

The substitution of the loop-T4 sequence of $\mathrm{G}_{4} \mathrm{~T}_{4} \mathrm{G}_{4}$ by AP sites [306] did not prevent the oligonucleotides from assembling into a dimeric, fold-back GQ either in $\mathrm{Na}^{+}$or in $\mathrm{K}^{+}$, although thermal stability of the unmodified GQ was found to be reduced. With the TBA GQ built by the GGTTGGTGTGGTTGG, in which the bold-underlined Ts were substituted with an AP site, one by one, the AP site in T9 considerably destabilized the native TBA GQ, whereas, the AP sites in the other positions moderately stabilized the unmodified TBA [307]. Depurination of a TTA loop (A/AP) of $A($ htel-21) also reduced the structural stability of the native GQ. The $\Delta T_{m}$ values ranged from -4.0 to $-6.7^{\circ} \mathrm{C}$ in $\mathrm{Na}^{+}$ and from -0.2 to $-6.5^{\circ} \mathrm{C}$ in $\mathrm{K}^{+}[293,308]$. These were much less drastic effects than those caused by the G/AP sites in the tetrads of the same GQ model [303]. Folding topology was, however, changed by the loop A/AP sites in a positiondependent way $[293,308]$. The wild-type A(htel-21) GQ is polymorphic in $\mathrm{K}^{+}$consisting of hybrid-1 and hybrid- 2 as well as $\mathrm{K}^{+}$-stabilized antiparallel structures [309]. The loss of a guanine in any G-tetrad shifted the equilibrium towards the antiparallel type [303], whereas, with two loop A/AP abasic GQs (A/AP in the first loop, abbreviated as ap7, and in third loop, ap19), a shift towards the formation of higher population of parallel strands in the mixture was observed. This means the shifting of conformational equilibrium towards higher concentrations of the hybrid folds. Actually, ap19 turned out to be a pure hybrid-2 type fold and the ap7 a hybrid1 architecture, although the latter formed clearly only at high, millimolar strand concentrations. Folding topology of the wild-type htel-21 and A(htel-21) GQs has been found to depend on the oligonucleotide strand concentrations and to change qualitatively above $2 \mathrm{mM}$ of strands [310-313]. Polymorphism increased when the A/AP was located in the middle loop at position 13 , and this was the ap13 structure(s). The A/AP site in the GQs of ap7 and ap19 was located in propeller-like loops. The abasic GQs transformed easier than the wild type into parallel GQs under dehydrating conditions (57\% ethanol). The GQ of A(htel-21), in which a loop adenine was replaced by an AP site, the ap7, ap13, and ap19, folded into parallel GQ in $\mathrm{K}^{+}$even in the absence of ethanol. This study [308] showed that the loop adenine AP sites are potent tools to fine-tune the folding arrangements of the telomeric GQs. The loop-abasic site-induced "monomorphism" has been confirmed by Wu et al. [314], who used this result for a sensor platform. They stated that an AP site replacing a loop adenine can extremely narrow the structure distribution to a specifically monomorphic GQ conformer, depending on the position of the AP site.

Loop thymidines have also been changed to AP sites. Rachwal and coworkers. [129] replaced the loop thymidines in the propeller-type single-base loops of the monomolecular parallel GQ assembled from the 17 -mer $\mathrm{T}\left(\mathrm{G}_{3} \mathrm{~T}\right)_{4}$. The substitution did not change the conformation; however, surprisingly, it increased the thermostability of the structure [129]. Another study by Esposito et al. [315] found that single AP sites replacing loop Ts in the GQs of the 16mer aptamer $\left(\right.$ GGGT) ${ }_{4}$ (T30923 or T30695) were not able to affect significantly the conformation and stability of the original structure. The original folding topology was a $5^{\prime}$ $5^{\prime}$ dimer of two stacked parallel GQs, each containing three G-tetrads and three single-thymidine reversed-chain loops. This aptamer GQ has been reported to exhibit anti-HIV activity by targeting the HIV integrase. With four such GQ analogs, authors could shed light on the steric interaction of the GQ with the integrase [315]. (A former study [20] also showed that the AP site-containing TBA GQs have altered biological activity: an AP site substituting for any $G$ residue of a G-tetrad in the TBA GQ significantly reduced the inhibition of thrombin. Substitution for either T4 or T13, which stack on the tetrad, also significantly diminished the inhibitory activity of the TBA GQ [20].) Sekridova et al. [316] studied the single-nucleotide loop-containing, twotetrad GQ of $\mathrm{G}_{2} \mathrm{AG}_{2} \mathrm{CG}_{2} \mathrm{AG}_{2}$ from the human ALU-repeat fragment, which was characterized by parallel topology and high thermodynamic stability. The loop bases were replaced with the flexible, nonnucleotide triethylene-glycol (teg) or a tetrahydrofuranyl AP site. The triethylene linker in $\left(G_{2} \text { teg }\right)_{3} G_{2}$ decreased $T_{m}$ of the native GQ significantly,

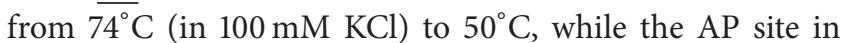
$\left(G_{2} \underline{A P}\right)_{3} G_{2}$, surprisingly, increased $T_{m}$ considerably, up to $85^{\circ} \mathrm{C}$. Furthermore, AFM (atomic force microscopy) data suggested that the two-tetrad GQ with the abasic loops was prone to dimerization in $10 \mathrm{mM} \mathrm{KCl}$. The dimers were supposed to be stacks of monomolecular GQs. At high concentration, $200 \mathrm{mM}$, of $\mathrm{KCl}$, this loop-abasic GQ formed higher-order aggregates, that is, short nanowires of $\sim 20 \mathrm{~nm}$ length, that were long G4-stacks [316]. The loop-abasic sites have been used in several other GQ model studies, such as in the ribo analog of the telomeric DNA and TERRA [169] and also with A(htel-21) [313]. Heddi et al. [317] investigated the formation of GQs containing $4 n-1$ guanines in the core and synthesized, among others, the $\mathrm{T}_{2} \mathrm{GGGT}\left(\mathrm{G}_{3} \mathrm{~T}\right)_{3}$ oligodeoxynucleotide, where the $\underline{G}$ is for the G/AP site in the middle tetrad of the three-tetrad GQ. The G/AP site drastically reduced the thermal stability of the unmodified parallel GQ, down to $51.2^{\circ} \mathrm{C}$ from $>85^{\circ} \mathrm{C}$ in $60 \mathrm{mM} \mathrm{K}^{+}$[318].

\subsection{Destabilizing Natural Base Lesions}

O6-Methylguanine $\left(m^{6} G\right)$. O6-Methylguanine is a major base lesion of nucleic acids in vivo [319], which is promutagenic and procarcinogenic [320]. The anti $\mathrm{m}^{6} \mathrm{dG}$ (Figure 15, 5) destabilized the tetramolecular GQ $\left[\mathrm{TG}_{5} \mathrm{~T}\right]_{4}$ in a positiondependent manner in $\mathrm{Na}^{+}$by forming $\mathrm{m}^{6} \mathrm{dG}$-tetrads, which did not change the parallel characteristics of the CD spectrum 
of the wild-type GQ [222]. Incorporated in place of each G at the $5^{\prime}$-end G-triplet of $\mathrm{AG}_{3}\left(\mathrm{TTAG}_{3}\right)_{3}$, which represented each of the three G-tetrads, the $\mathrm{m}^{6} \mathrm{G}$ reduced the thermal stability of the unmodified GQ. The largest destabilizing effect was observed with the middle tetrad substitution, both in $\mathrm{Na}^{+}$and in $\mathrm{K}^{+}$. CD spectrum of the unmodified 22mer in $\mathrm{Na}^{+}$was only slightly modified by $\mathrm{m}^{6} \mathrm{G}$. In $\mathrm{K}^{+}$, the unmodified oligonucleotide formed hybrid-type mixed folds but the spectra of the outer tetrads-modified GQs looked like their $\mathrm{Na}^{+}$-spectra. Spectrum of the middle tetrad-modified structure was qualitatively different from the others. The decreased thermal stability might have been the consequence of missing cation coordination, the disrupted circular $\mathrm{H}$ bonding, and the reduced stacking interactions [321]. Bulky substituents linked to $\mathrm{O} 6$ of G, such as the O6-p-nitrobenzyl group, completely hindered the formation of GQ from the TBA oligodeoxynucleotide when substituted at G1. Adding $\mathrm{Na}_{2} \mathrm{~S}_{2} \mathrm{O}_{4}$ to the solution of the modified sequence resulted in the formation of $\mathrm{GQ}$, as the $\mathrm{CD}$ spectra showed, due to the removal of the reduction-sensitive p-nitrobenzyl group [322]. $\mathrm{m}^{6} \mathrm{G}$ is one of the few natural base lesions that was found so far to be repaired. O6-Alkylguanine-DNA alkyltransferase (AGT) is the repair enzyme that removes the promutagenic O6-alkylguanine adducts from duplex DNA in vitro and in vivo. Using $\mathrm{m}^{6} \mathrm{G}$-substituted $\mathrm{A}$ (htel-21) GQs, Hellman et al. [259] found that AGT repaired the $m^{6} G$ adducts located within the folded GQ, and the rate of repair was comparable to those found with duplex DNAs under analogous conditions. Repair was dependent on the position of the adduct. In general, $\mathrm{m}^{6} \mathrm{G}$ located in the outermost (top and bottom) tetrads of a GQ stack exhibited more rapidphase repair than did the adducts located in the inner tetrad.

Hypoxanthine (I for Inosine). Enzymatic deamination of adenine nucleosides of RNA and DNA in vivo leads to hypoxanthine-ribosides, that is, to inosine or to $2^{\prime}$ deoxyinosine [299, 323]. Hypoxanthine, I (Figure 15, 6), is a $G$ analog with a missing 2-amino group, and thus the Hoogsteen-type circular double H-bonded G-tetrad loses a hydrogen bond if $\underline{I}$ is formed or incorporated into a GQ. This is supposed to lead to some destabilization of the GQ. The I base was first incorporated into the Oxytricha telomere repeat $\mathrm{G}_{4} \mathrm{~T}_{4} \mathrm{G}_{4}$ sequence in 1993. The $\mathrm{G}_{4} \mathrm{~T}_{4} \mathrm{G}_{3} \mathrm{I}$ formed a diagonally looped symmetrical bimolecular antiparallel GQ with four Gtetrads and T4-loops in both $\mathrm{Na}^{+}$and $\mathrm{K}^{+}$[324]. Later, $\underline{\mathrm{I}}$ and $\mathrm{U}$ were both inserted into the Oxy-3.5 sequence $\mathrm{G}_{4}\left(\mathrm{~T}_{4} \mathrm{G}_{4}\right)_{3}$ that resulted in $\mathrm{G}_{4}$ TUTUG $_{4} \mathrm{~T}_{4} \mathrm{G}_{4}$ UUTTG $_{3}$ I. Both the wild-type and the modified sequences formed a monomolecular GQ with a diagonal T4 loop and two modified edgewise loops. Thermodynamic data were not published [224]. Tetramolecular structures were also investigated for the effect of hypoxanthine. The I-tetrads drastically destabilized the parallel GQs of $\left[\mathrm{TG}_{4} \mathrm{~T}\right]_{4}$ and $\left[\mathrm{TG}_{5} \mathrm{~T}\right]_{4}$ in position-dependent ways [222]. I-containing tetramolecular GQs were also built from the single repeat htel sequences TTAGGIT and TTAGGGIT and analyzed by $1 \mathrm{H}$ NMR [325]. Numerous intramolecular GQ-forming htel sequences were also substituted with hypoxanthine. Relative stability of an I-modified htel-21 GQ was different in $\mathrm{Na}^{+}$and $\mathrm{K}^{+}$solutions. In position-dependent ways, the $\Delta T_{m}$ values were $-10^{\circ} \mathrm{C}$ or more negative by single substitutions and $-20^{\circ} \mathrm{C}$ or lower by double substations in $\mathrm{K}^{+}$, and $-6.5^{\circ} \mathrm{C}$ or larger on single and $-14^{\circ} \mathrm{C}$ or larger on double substitutions in $\mathrm{Na}^{+}$, as determined by Risitano and Fox [326]. With the GQ of $\left(\mathrm{TAG}_{3}\right)_{3} \mathrm{TAI} G \mathrm{I}, \underline{\mathrm{I}}$ did not change the $\mathrm{CD}$ signature of the wild-type antiparallel GQ [327], and similarly, the single I in the GQ of GIGT(GGGT) did not change the parallel CD spectrum of $(\mathrm{GGGT})_{4}$ but made the GQ structure more stable for NMR studies by significantly improving the spectral resolution $[328,329]$. The absence of the 2-amino group facilitates the folding transition of the unstable or equilibrium structures into another fold; that is, substitution of a dG by dI could selectively disfavor particular GQ conformations. An example for this was the study that used the $\mathrm{dI}$ for $\mathrm{dG}$ substitution to change the conformational equilibrium between competing GQ folds in $\mathrm{AG}_{4} \mathrm{AG}_{4} \mathrm{CTG}_{3} \mathrm{AG}_{3} \mathrm{C}[330]$. The G-to-I substitution at various $G$ positions in the five TTAGGG repeat htel GQs resulted in the protruding of the GIG section into a long loop. Thermal stability of the unmodified htel- 25 was $62^{\circ} \mathrm{C}$, which the extended GIG repeats decreased significantly. $T_{m}$ of the htel31 (I at pos.22) was $49.8^{\circ} \mathrm{C}$, that of the double-modified htel$37\left(\right.$ I22, I28) was $47.7^{\circ} \mathrm{C}$, and that of the triple-modified htel$43(\mathrm{I} 22, \mathrm{I} 28, \mathrm{I} 34)$ was $46.5^{\circ} \mathrm{C}$ [81]. With another long repeat sequence, the double G-to-I substituted $\mathrm{TAG}_{3}\left(\mathrm{TTAG}_{3}\right)_{7} \mathrm{TT}$, it was found that one permutation of I-substitutions at the $5^{\prime}$-end produced a minor increase in the thermodynamic stability relative to the wild type, while four other double mutations, however, decreased it, some of them significantly [331]. G-to-I substitutions were also used with GQs of A(htel21) and $\mathrm{A}\left(\right.$ htel-21)T [332], the VEGFA $\mathrm{G}_{3} \mathrm{AG}_{3} \mathrm{TTG}_{4} \mathrm{TG}_{3}$, the c-myc Pul8 $\mathrm{AG}_{3} \mathrm{TG}_{4} \mathrm{AG}_{3} \mathrm{TG}_{4}$, and the PIM1 $\mathrm{G}_{3} \mathrm{CG}_{4} \mathrm{CG}_{5} \mathrm{CG}_{4}$ sequences [333]. Guanine in the central TGT loop of the TBA GQ was replaced by adenine or hypoxanthine to study the role of G8 in the GQ stabilization. The G-to-I substitution resulted in a slight decrease in the thermal stability; the Gto-A change triggered a larger destabilization. $T_{m}$ values were $50.8^{\circ} \mathrm{C}, 49.5^{\circ} \mathrm{C}$, and $45.0^{\circ} \mathrm{C}$ for the wild-type, the I-modified, and the A-modified GQs, respectively [334]. Results of a recent study [335] have shown that incorporation of $\mathrm{dI}$ into selected positions of AS1411 GQ can elevate its biological activity. AS1411 is a 26-mer GQ-forming aptamer with antiproliferative activity originating from selective binding to nucleolin protein. The augmented activity is thought to arise from stronger binding to the nucleolin. Based simply on the increasing amplitude of the $264 \mathrm{~nm}$ CD peak by $\mathrm{dI}$ substitutions, authors concluded that dI may also stabilize the GQ [335].

Xanthine $(X)$. Xanthine $(\mathrm{X})$ or 2,6-dioxopurine or 3,7dihydropurine-2,6-dione (Figure 15,7), is an analog of guanine. It is found in all human tissues and is also a natural base derivative in DNA originating from guanine by guanine deaminase or from hypoxanthine by xanthine oxidoreductase [299]. Similar to inosine, xanthine also disrupts the Hoogsteen-type circular double H-bonding structure of a Gtetrad due to the missing $\mathrm{NH}_{2}$ group of $\mathrm{G}$, which probably leads to a destabilized GQ fold. Not counting the $0^{8} \mathrm{G}-\mathrm{X}$ double modifications $[282,283,288]$, the $\mathrm{X}$ in GQ tetrads has 

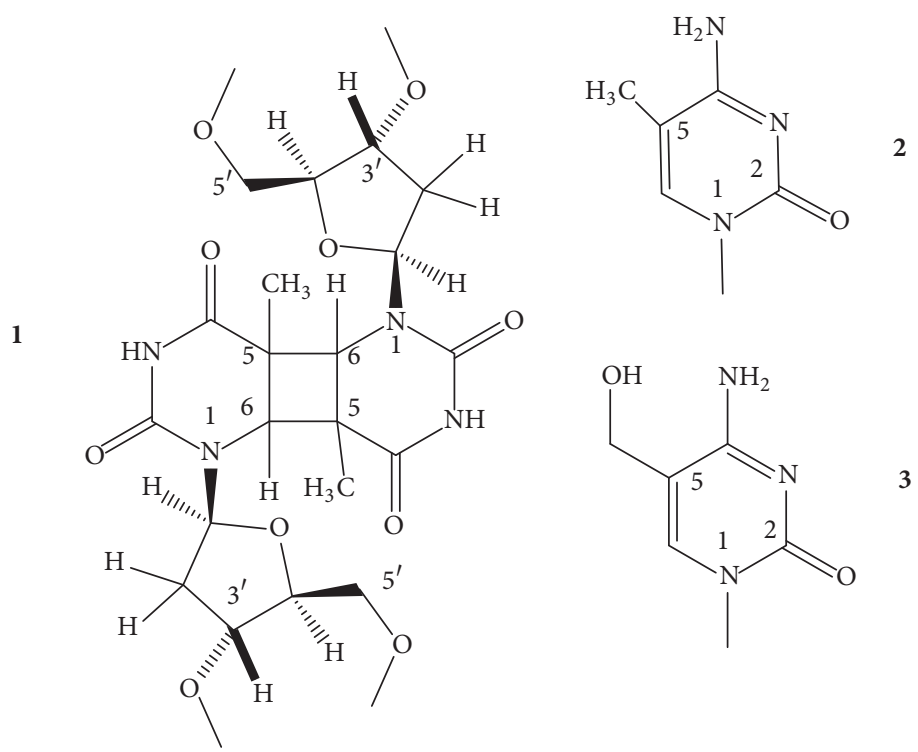

2

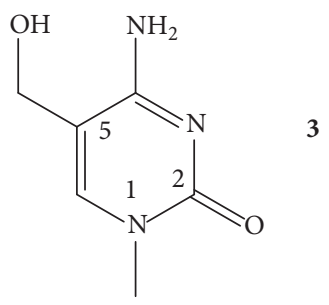

FIGURE 16: Structure of the anti cyclobutane thymine dimer (1), 5-methylcytosine (2), and the 5-hydroxymethylcytosine (3).

only been studied via in silico methods [336, 337]. A complex quantum chemical approach showed a high degree of structural and energetic compatibility between the guanine and xanthine-based GQ models. The calculations established that hydrogen bonding made the greatest $(\sim 50 \%)$ contribution to the internal stability of the DNA GQs. Base stacking and ion coordination terms proved commensurable and accounted for the rest of the energetic contributions. Using two- or three-tetrad model systems, the guanine tetrad structures benefited from the high degree of $\mathrm{H}$-bond cooperativity while the xanthine tetrad models were characterized by the more favorable stacking interactions [338]. Experimental proofs for these conclusions are not yet available.

Cyclobutane Thymine Dimers. Ultraviolet B (UVB) light irradiation induces the formation of cyclobutane pyrimidine dimers in nucleic acids in vivo. Under physiologically relevant solution conditions, the UVB light can prompt the formation of anti cyclobutane thymine dimers (Figure 16, 1) in GQs, as Smith et al. recently reported [339]. The anti refers to the position of the 5-methyl groups of the two thymines of the cyclobutane ring. Stereochemistry and yield of formation greatly depended on the topology of the GQ, the cations present, and the temperature of the solution. The two-tetrad basket-type model formed from various mutated analogs of the 26-mer $A_{3}$ (htel-21) $A_{2}$, in which two-tetrad fold was first described for the (htel-21) T in $\mathrm{K}^{+}$by Phan's group [77], was found as the first GQ structure in which the thymine dimer could form in $\mathrm{K}^{+}$, but not in $\mathrm{Na}^{+}$. The T-dimer formed between the two edgewise loops. In this fold, the loop thymines are close enough for the UVB-induced cyclization reaction to occur. Other dynamic $\mathrm{K}^{+}$-topologies were also proposed as appropriate stereo structures for T-dimer formation. Thermal stability and conformational changes caused by the dimer formation were discussed by the authors [339].
Clustered Lesions in GQs. Ionizing radiation produces clustered lesions in the genetic material that results in distorted secondary structures in the double-stranded DNA. Clustered lesions have been found to be more difficult to repair, which were thus more harmful than the single, isolated ones and were detrimental to cells [340]. By definition, clustered DNA lesions form when two or more forms of damage appear in the double-stranded DNA strands within one to two helical turns, which involves 10-20 base pairs [341]. This type of lesions can also form in GQs. Sagi and coworkers [342] described for the first time the effect of clustered lesions on GQ structures, specifically clustered loop adenine (A/AP), tetrad guanine (G/AP), and A/AP + G/AP abasic sites (AP sites are among the most frequent natural lesions [299]). We have reformulated the definition described for duplex DNAs to be suitable for GQs as well: two or more lesions within a four-stranded, two- or three-tetrad core GQ unit consisting of up to 21 nucleotides in the core. For the experiments, the potential GQ-forming A(htel-21) and TA(htel-21) TT oligodeoxynucleotides were synthesized with 36 permutations of double and triple tetrahydrofuranyl AP sites. In $100 \mathrm{mM} \mathrm{K}^{+}$solution double loop A/AP sites destabilized the wild-type A(htel-21) GQ $\left(T_{m} 66.1^{\circ} \mathrm{C}\right)$ by $6-8^{\circ} \mathrm{C}$, whereas, when each of the three TTA loops contained an A/AP or one TTA loop was replaced by three A/AP sites, the wild type was even moderately stabilized, by $1-$ $2^{\circ} \mathrm{C}$. The unmodified GQ of TA(htel-21)TT $\left(T_{m}: 62.0^{\circ} \mathrm{C}\right)$ was extensively destabilized by single tetrad G/APs, by 15 to $36^{\circ} \mathrm{C}$; the latter figure belonged to the middle tetrad substitutions of the three-tetrad GQ. Depending on its position, the single A/APs diminished or intensified the damaging effect of G/APs. The largest negative effects were observed when the G/AP was in the middle tetrad: only partially folded structures were formed at $37^{\circ} \mathrm{C}$. Half of the 21 variants of double and triple clustered G/AP sites led to more or 
less unfolded structures at $37^{\circ} \mathrm{C}$; others had $T_{m}$ s between 31 and $37^{\circ} \mathrm{C}$, meaning partial folding only. Three of those oligodeoxynucleotides containing one G/AP in the middle tetrad remained unstructured. The single to triple A/APs in the loops increased the population of parallel strands in their structures, thus shifting the antiparallel $\mathrm{A}(\mathrm{htel}-21)$ GQs into hybrid or parallel forms. Single G/APs and thus also the G/AP plus A/AP combinations, however, inhibited the formation of parallel strands and these GQs adopted antiparallel topologies. CD spectra matched those of the $\mathrm{Na}^{+}$stabilized htel GQs. The in vitro results may suggest that formation of clustered lesions in the chromosome-capping structure, in the Shelterin complex, can induce unfolding of existing GQ structures, which, in turn, can lead to telomere shortening [342].

\subsection{Epigenetic Modifications}

5-Methyl- and 5-Hydroxymethylcytosines $\left(m^{5} \mathrm{C}\right.$ and $\left.h m^{5} \mathrm{C}\right)$. Epigenetic 5-methylation of cytosine is among the processes that control the gene expression in human cells, which has also associations with the development of cancer and other diseases [343-345]. Methylation usually occurs at $\mathrm{CpG}$ sites and $70-80 \%$ of the CpG sites in mammalian cells are methylated. $\mathrm{m}^{5} \mathrm{C}$ accounts for $\sim 1 \%$ of all DNA bases in the human genome $[344,346]$. The methylation is a reversible enzymatic process [347]. Demethylation of $\mathrm{m}^{5} \mathrm{C}$ (Figure 16, 2) follows stepwise through 5-hydroxymethylcytosine $\left(\mathrm{hm}^{5} \mathrm{C}\right.$ ) (Figure 16, 3), to 5-formylcytosine and finally to 5 -carboxylcytosine, and this base is then excised by the subsequent repair processes. Interestingly, all these bases have been found to occur in mammalian embryonic stem (ES) cells [348], indicating that the modified cytosines are stable and may themselves have some role in gene regulation. Their abundance in cells is very low, $0.5 \%, \sim 0.002 \%$, and $0.0003 \%$, respectively, for the three oxidized derivatives of $\mathrm{m}^{5} \mathrm{C}$ in mouse ES cells [349]. Their level also depends on age, at least in the brain [350]. Regarding the presence of $\mathrm{m}^{5} \mathrm{C}$ in $\mathrm{CpG}$ sites of GQs, the P1 promoter sequence of bcl-2 oncogene, the $\mathrm{CG}_{3} \mathrm{CGCG}_{3} \mathrm{AG}_{2} \mathrm{~A}_{2} \mathrm{G}_{5} \mathrm{CG}_{3} \mathrm{AGC}$ was first examined, where the cytosines at $\mathrm{Cl}, \mathrm{C} 5, \mathrm{C} 7$, and $\mathrm{C} 21$ positions were methylated. The methylations induced the formation of GQ in the P1 promoter by greatly increasing the thermal stability of the structure. Methylation also protected the GQ from unfolding by the complementary strand. The proposed mechanism explained the transcription inactivation by hypermethylation that induces formation of GQ from duplex in the promoter and the gene activation by hypomethylation [351]. The 5hydroxymethylated cytosines $\left(\mathrm{hm}^{5} \mathrm{C}\right)$ are also involved in the regulation of gene expression of certain genes. The studies described by Molnar et al. [352] characterized the effects of $\mathrm{hm}^{5} \mathrm{C}$-modified $\mathrm{CpG}$ islands on the GQ (and the i-motif) structures. Single $\mathrm{hm}^{5} \mathrm{C}$ was incorporated into the G-rich (and C-rich) sequences from the vascular endothelial growth factor (VEGF) promoter. The results indicated that two of the three $\mathrm{hm}^{5} \mathrm{C}$-containing loops showed a significant decrease in thermal stability of the GQ (on the contrary, thermal stability of the i-motif increased somewhat by the $\mathrm{hm}^{5} \mathrm{C}$ ) [352]. Another study was carried out with the G-rich and
C-rich strand models of the C9orf72 repeat, the (GGGGCC) 8 and (GGCCCC $)_{8}$ DNA oligonucleotides, to investigate the effect of epigenetic signals on the macromolecular structure. Each $\mathrm{C}$ was replaced by $\mathrm{m}^{5} \mathrm{C}$ or $\mathrm{hm}^{5} \mathrm{C}$ at each $\mathrm{CpG}$ motive. The G-rich strands formed G-tetrad- and various mixed G $+\mathrm{C}$ tetrad-containing unusually long intramolecular chairtype antiparallel GQs. GQs of the C-rich strands contained $C$ tetrads and also mixed $G+C$ tetrads while no i-motif was observed. The $\mathrm{m}^{5} \mathrm{C}$ moderately increased, by $1.2^{\circ} \mathrm{C}$, while $\mathrm{hm}^{5} \mathrm{C}$ considerably decreased, by $4.7^{\circ} \mathrm{C}$, the thermal stability of the unmodified intramolecular GQ of the G-rich strand in $\mathrm{K}^{+}$[353].

8-Oxoguanine $\left(o^{8} G\right)$. ROS, the reactive forms of oxygen, are free radicals that have unpaired electrons in their outer orbits. ROS can cause significant damage in cells, where DNA is one of the major targets. Recent studies, however, also demonstrated that ROS have normal physiological functions as well. For instance, $o^{8} \mathrm{G}$ (Figure 15, 1), one of the main initial damage products of ROS, has recently been described by Fleming et al. [354] as it is also an epigenetic modification in gene promoters that regulate the transcription via the repair process of the base excision repair (BER). Gene expression was found to occur when $o^{8} \mathrm{G}$ is formed in the G-rich, potentially GQ-forming DNA sequences. The damage initiated the action of the BER by the 8-oxo- $2^{\prime}$-deoxyguanosine DNA glycosylase (hOGG1), whose reaction yields an abasic (AP) site. The AP site enabled unwinding (melting) of the duplex DNA that facilitated the conversion of the G-rich strand to a GQ fold. These reactions finally resulted in the activation of the VEGF or NTHL1 genes. The authors identified 61 human DNA repair genes that might be activated by this mechanism [354]. Later, Fleming and Burrows have confirmed these findings and also compared the epigenetic pathway of $0^{8} \mathrm{G}$ with that of another base analog, the 5-methylcytosine [355].

\section{Concluding Remarks}

This study illustrates the established, widespread use of synthetic nucleotide analogs in the structural studies of the most researched noncanonical nucleic acid structures, the GQs. Application of the discussed, known analogs and introduction of new derivatives is not going to stop; thus we can expect further growth of the field. Significant advances can be anticipated in the elucidation of the effect of numerous, unexplored single and clustered natural base lesions on the structures and repair of GQs. Recent findings of epigenetics on the role of the formation of some oxidative DNA base lesions followed by conformation switching of the G-rich duplex DNAs to GQs suggest the opening of a new area in the gene expression research.

\section{Abbreviations}

GQ: G-quadruplex

$T_{m}$ : Half-way temperature of the thermal unfolding profile of the GQs

htel: Human telomere repeat sequences 
TBA: Thrombin-binding aptamer

CD: Circular dichroism.

\section{Additional Points}

In most cases, DNA GQs are discussed here, and therefore the " $d$ " is omitted from the sequence; for instance, $d$ (TGGGAG) is denoted as TGGGAG, or the htel DNA, such as the $\mathrm{d}\left[\mathrm{G}_{3}\left(\mathrm{TTAG}_{3}\right)_{3}\right]$, is denoted as $\mathrm{G}_{3}\left(\mathrm{TTAG}_{3}\right)_{3}$ or in an even shorter way as htel-21. The $5^{\prime}$ - or $3^{\prime}$-extended htel DNA oligonucleotides of the basic 21-nucleotide-long (21-mer) htel-21 are also abbreviated as, for example, TA(htel-21)TT that refers to $\mathrm{d}\left[\mathrm{TAG}_{3}\left(\mathrm{TTAG}_{3}\right)_{3} \mathrm{TT}\right]$. The bimolecular and tetramolecular GQs are abbreviated by using square brackets, such as with [TGGGT] $]_{4}$. RNA GQ sequences are abbreviated with small letters, like ugggu.

\section{Conflicts of Interest}

The author declares that there are no conflicts of interest.

\section{References}

[1] B. Saccà, L. Lacroix, and J.-L. Mergny, "The effect of chemical modifications on the thermal stability of different Gquadruplex-forming oligonucleotides," Nucleic Acids Research, vol. 33, no. 4, pp. 1182-1192, 2005.

[2] S. Neidle and S. Balasubramanian, Quadruplex Nucleic Acids, Royal Society of Chemistry, Cambridge, UK, 2006.

[3] A. Aviñó, C. Fàbrega, M. Tintoré, and R. Eritja, “Thrombin binding aptamer, more than a simple aptamer: chemically modified derivatives and biomedical applications," Current Pharmaceutical Design, vol. 18, no. 14, pp. 2036-2047, 2012.

[4] O. Doluca, J. M. Withers, and V. V. Filichev, "Molecular engineering of guanine-rich sequences: Z-DNA, DNA triplexes, and G-quadruplexes," Chemical Reviews, vol. 113, no. 5, pp. 3044-3083, 2013.

[5] J. Sagi, "G-quadruplexes incorporating modified constituents: A review," Journal of Biomolecular Structure and Dynamics, vol. 32, no. 3, pp. 477-511, 2014.

[6] J. B. Chaires and D. Graves, Topics in Current Chemistry, vol. 330, Springer, 2013.

[7] A. Varizhuk, N. Ilyinsky, I. Smirnov, and G. Pozmogova, "G4 aptamers: Trends in structural design," Mini-Reviews in Medicinal Chemistry, vol. 16, no. 16, pp. 1321-1329, 2016.

[8] J. Sagi, "Designing G-quadruplex topologies by focusing on sequence modifications," in G-Quadruplex Structures, Formation and Role in Biology, H. Santos, Ed., pp. 1-51, Nova Science Publishers, New York, NY, USA, 2016.

[9] A. Virgilio, V. Esposito, R. Filosa, L. Mayol, and A. Galeone, "The introduction of inversion of polarity sites in DNA Gquadruplex structures: Effects and perspectives," Mini-Reviews in Medicinal Chemistry, vol. 16, no. 7, pp. 509-523, 2016.

[10] E. Zavialova and A. Kopylov, "G-quadruplexes and I-motifs as scaffolds for molecular engineering of DNA aptamers," in GQuadruplex Structures, Formation and Role in Biology, $\mathrm{H}$. Santos, Ed., pp. 53-80, Nova Science Publishers, Inc, New York, NY, USA, 2016.

[11] P. L. Tran, A. De Cian, J. Gros, R. Moriyama, and J. Mergny, "Tetramolecular Quadruplex Stability and Assembly," in
Quadruplex Nucleic Acids, vol. 330 of Topics in Current Chemistry, pp. 243-273, Springer Berlin Heidelberg, Berlin, Heidelberg, 2013.

[12] V. Esposito, A. Randazzo, A. Messere et al., "Synthesis and structural characterization of PNA-DNA quadruplex-forming chimeras," European Journal of Organic Chemistry, no. 17, pp. 3364-3371, 2003.

[13] P. E. Nielsen and D. H. Appella, Peptide Nucleic Acids: Methods and Protocols, Humana Press, 2nd edition, 2014.

[14] T. Agarwal, D. Pradhan, I. Géci et al., "Improved inhibition of telomerase by short twisted intercalating nucleic acids under molecular crowding conditions.," Nucleic acid therapeutics, vol. 22, no. 6, pp. 399-404, 2012.

[15] M. Prokofjeva, V. Tsvetkov, D. Basmanov et al., "Anti-HIV Activities of Intramolecular G4 and Non-G4 Oligonucleotides," Nucleic Acid Therapeutics, vol. 27, no. 1, pp. 56-66, 2017.

[16] D. Musumeci, C. Riccardi, and D. Montesarchio, "G-Quadruplex forming oligonucleotides as Anti-HIV agents," Molecules, vol. 20, no. 9, pp. 17511-17532, 2015.

[17] A. Aviñó, G. Portella, R. Ferreira et al., "Specific loop modifications of the thrombin-binding aptamer trigger the formation of parallel structures," FEBS Journal, vol. 281, no. 4, pp. 1085-1099, 2014.

[18] R. F. Macaya, P. Schultze, F. W. Smith, J. A. Roe, and J. Feigon, "Thrombin-binding DNA aptamer forms a unimolecular quadruplex structure in solution," Proceedings of the National Academy of Sciences of the United States of America, vol. 90, no. 8, pp. 3745-3749, 1993.

[19] K. Y. Wang, S. McCurdy, R. G. Shea, S. Swaminathan, and P. H. Bolton, "A DNA aptamer which binds to and inhibits thrombin exhibits a new structural motif for DNA," Biochemistry, vol. 32, no. 8, pp. 1899-1904, 1993.

[20] K. Y. Wang, S. H. Krawczyk, N. Bischofberger, S. Swaminathan, and P. H. Bolton, "The tertiary structure of a DNA aptamer which binds to and inhibits thrombin determines activity," Biochemistry, vol. 32, no. 42, pp. 11285-11292, 1993.

[21] S. R. Nallagatla, B. Heuberger, A. Haque, and C. Switzer, "Combinatorial synthesis of thrombin-binding aptamers containing iso-guanine," Journal of Combinatorial Chemistry, vol. 11, no. 3, pp. 364-369, 2009.

[22] P. A. Rachwal and K. R. Fox, "Quadruplex melting," Methods, vol. 43, no. 4, pp. 291-301, 2007.

[23] J.-L. Mergny and L. Lacroix, Current Protocols in Nucleic Acid Chemistry, John Wiley \& Sons, Inc., Hoboken, NJ, USA, 2009.

[24] B. Pagano, A. Randazzo, I. Fotticchia, E. Novellino, L. Petraccone, and C. Giancola, "Differential scanning calorimetry to investigate G-quadruplexes structural stability," Methods, vol. 64, no. 1, pp. 43-51, 2013.

[25] J. M. Dettler, R. Buscaglia, V. H. Le, and E. A. Lewis, "DSC deconvolution of the structural complexity of c-MYC P1 promoter G-Quadruplexes," Biophysical Journal, vol. 100, no. 6, pp. 1517-1525, 2011.

[26] M. Vorlícková, M. Tomasko, A. J. Sagi, K. Bednarova, and J. Sagi, "8-Oxoguanine in a quadruplex of the human telomere DNA sequence," FEBS Journal, vol. 279, no. 1, pp. 29-39, 2012.

[27] X. Liu, I. C. M. Kwan, S. Wang, and G. Wu, "G-quartet formation from an N2-modified guanosine derivative," Organic Letters, vol. 8, no. 17, pp. 3685-3688, 2006.

[28] M. S. Kaucher and J. T. Davis, "N2, C8-disubstituted guanosine derivatives can form G-quartets," Tetrahedron Letters, vol. 47, no. 36, pp. 6381-6384, 2006. 
[29] M. Nakano, H. Tateishi-Karimata, S. Tanaka et al., "Thermodynamic properties of water molecules in the presence of cosolute depend on DNA structure: A study using grid inhomogeneous solvation theory," Nucleic Acids Research, vol. 43, no. 21, pp. 10114-10125, 2015.

[30] S.-I. Nakano, D. Miyoshi, and N. Sugimoto, "Effects of molecular crowding on the structures, interactions, and functions of nucleic acids," Chemical Reviews, vol. 114, no. 5, pp. 2733-2758, 2014.

[31] H. Hotoda, M. Koizumi, R. Koga et al., "Biologically active oligodeoxyribonucleotides, 5. 5'-end-substituted d(TGGGAG) possesses anti-human immunodeficiency virus type 1 activity by forming a G-quadruplex structure," Journal of Medicinal Chemistry, vol. 41, no. 19, pp. 3655-3663, 1998.

[32] M. Koizumi, K. Akahori, T. Ohmine et al., "Biologically active oligodeoxyribonucleotides. Part 12:1 N2-methylation of 2'deoxyguanosines enhances stability of parallel G-quadruplex and anti-HIV-1 activity," Bioorganic and Medicinal Chemistry Letters, vol. 10, no. 19, pp. 2213-2216, 2000.

[33] G.-X. He, S. H. Krawczyk, S. Swaminathan et al., "N2- and C8-substituted oligodeoxynucleotides with enhanced thrombin inhibitory activity in vitro and in vivo," Journal of Medicinal Chemistry, vol. 41, no. 13, pp. 2234-2242, 1998.

[34] C. J. Lech and A. T. Phan, "Ball with hair: modular functionalization of highly stable G-quadruplex DNA nano-scaffolds through N2-guanine modification," Nucleic Acids Research, vol. 45, no. 11, pp. 6265-6274, 2017.

[35] F. Seela, C. Wei, and A. Melenewski, "Isoguanine quartets formed by d(T4isoG4T4): Tetraplex identification and stability," Nucleic Acids Research, vol. 24, no. 24, pp. 4940-4945, 1996.

[36] F. Seela and C. Wei, "7-Deazaisoguanine quartets: Selfassembled oligonucleotides lacking the Hoogsteen motif," Chemical Communications, no. 19, pp. 1869-1870, 1997.

[37] C. Roberts, J. C. Chaput, and C. Switzer, "Beyond guanine quartets: Cation-induced formation of homogenous and chimeric DNA tetraplexes incorporating iso-guanine and guanine," Chemistry and Biology, vol. 4, no. 12, pp. 899-908, 1997.

[38] J. C. Chaput and C. Switzer, "A DNA pentaplex incorporating nucleobase quintets," Proceedings of the National Academy of Sciences of the United States of America, vol. 96, no. 19, pp. 1061410619, 1999.

[39] F. Seela and R. Kröschel, "Quadruplex and pentaplex selfassemblies of oligonucleotides containing short runs of 8-aza-7deaza-2I-deoxyisoguanosine or 2/-deoxyisoguanosine," Bioconjugate Chemistry, vol. 12, no. 6, pp. 1043-1050, 2001.

[40] B. A. Rosenzweig, N. T. Ross, D. M. Tagore, J. Jayawickramarajah, I. Saraogi, and A. D. Hamilton, "Multivalent protein binding and precipitation by self-assembling molecules on a DNA pentaplex scaffold," Journal of the American Chemical Society, vol. 131, no. 14, pp. 5020-5021, 2009.

[41] N. Shumayrikh, Y. C. Huang, and D. Sen, "Heme activation by DNA: Isoguanine pentaplexes, but not quadruplexes, bind heme and enhance its oxidative activity," Nucleic Acids Research, vol. 43, no. 8, pp. 4191-4201, 2015.

[42] S. Bazzi, J. Novotný, Y. P. Yurenko, and R. Marek, "Designing a New Class of Bases for Nucleic Acid Quadruplexes and Quadruplex-Active Ligands," Chemistry-A European Journal, vol. 21, no. 26, pp. 9414-9425, 2015.

[43] L. Petraccone, E. Erra, V. Esposito et al., "Biophysical properties of quadruple helices of modified human telomeric DNA," Biopolymers, vol. 77, no. 2, pp. 75-85, 2005.
[44] V. Esposito, A. Randazzo, L. Petraccone et al., "Molecular modeling studies of a parallel stranded quadruplexes containing a 8-bromoadenosine," Nucleosides, Nucleotides and Nucleic Acids, vol. 24, no. 5-7, pp. 789-794, 2005.

[45] B. Catalanotti, A. Galeone, L. Gomez-Paloma, L. Mayol, and A. Pepe, '2'-Deoxy-8-(propyn-1-yl)adenosine-containing oligonucleotides: Effects on stability of duplex and quadruplex structures," Bioorganic and Medicinal Chemistry Letters, vol. 10, no. 17, pp. 2005-2009, 2000.

[46] A. Randazzo, V. Esposito, A. Galeone, M. Varra, A. Virgilio, and L. Mayol, "Synthesis and structural study of quadruplex structures containing 2/-deoxy-8-methyladenosine," Nucleosides, Nucleotides and Nucleic Acids, vol. 24, no. 5-7, pp. 539-543, 2005.

[47] A. Virgilio, V. Esposito, A. Randazzo, L. Mayol, and A. Galeone, "Effects of 8-methyl-2I-deoxyadenosine incorporation into quadruplex forming oligodeoxyribonucleotides," Bioorganic and Medicinal Chemistry, vol. 13, no. 4, pp. 1037-1044, 2005.

[48] A. Virgilio, L. Petraccone, V. Vellecco et al., "Site-specific replacement of the thymine methyl group by fluorine in thrombin binding aptamer significantly improves structural stability and anticoagulant activity," Nucleic Acids Research, vol. 43, no. 22, pp. 10602-10611, 2015.

[49] V. Esposito, A. Pepe, R. Filosa, L. Mayol, A. Virgilio, and A. Galeone, "A novel pyrimidine tetrad contributing to stabilize tetramolecular G-quadruplex structures," Organic and Biomolecular Chemistry, vol. 14, no. 10, pp. 2938-2943, 2016.

[50] G. Paragi, Z. Kupihár, G. Endre, C. Fonseca Guerra, and L. Kovács, "The evaluation of 5-amino- and 5-hydroxyuracil derivatives as potential quadruplex-forming agents," Organic and Biomolecular Chemistry, vol. 15, no. 10, pp. 2174-2184, 2017.

[51] H. Tateishi-Karimata, T. Muraoka, K. Kinbara, and N. Sugimoto, "G-Quadruplexes with Tetra(ethylene glycol)-Modified Deoxythymidines are Resistant to Nucleases and Inhibit HIV-1 Reverse Transcriptase," ChemBioChem, pp. 1399-1402, 2016.

[52] H. Tateishi-Karimata, T. Ohyama, T. Muraoka et al., "Newly characterized interaction stabilizes DNA structure: oligoethylene glycols stabilize G-quadruplexes $\mathrm{CH}-\pi$ interactions," Nucleic Acids Research, vol. 45, no. 12, pp. 7021-7030, 2017.

[53] A. A. Tanpure and S. G. Srivatsan, "Conformation-sensitive nucleoside analogues as topology-specific fluorescence turn-on probes for DNA and RNA G-quadruplexes," Nucleic Acids Research, vol. 43, no. 22, article e149, 2015.

[54] J.-Y. Liao, I. Anosova, S. Bala, W. D. Van Horn, and J. C. Chaput, "A parallel stranded G-quadruplex composed of threose nucleic acid (TNA)," Biopolymers, vol. 107, no. 3, Article ID e22999, 2017.

[55] N. Borbone, M. Bucci, G. Oliviero et al., "Investigating the role of $\mathrm{T} 7$ and $\mathrm{T} 12$ residues on the biological properties of thrombinbinding aptamer: Enhancement of anticoagulant activity by a single nucleobase modification," Journal of Medicinal Chemistry, vol. 55, no. 23, pp. 10716-10728, 2012.

[56] M. Scuotto, M. Persico, M. Bucci et al., "Outstanding effects on antithrombin activity of modified TBA diastereomers containing an optically pure acyclic nucleotide analogue," Organic and Biomolecular Chemistry, vol. 12, no. 28, pp. 5235-5242, 2014.

[57] L. J. Aaldering, V. Poongavanam, N. Langkjær et al., "Development of an Efficient G-Quadruplex-Stabilised Thrombin-Binding Aptamer Containing a Three-Carbon Spacer Molecule," ChemBioChem, vol. 18, no. 8, pp. 755-763, 2017.

[58] S. De Tito, F. Morvan, A. Meyer et al., "Fluorescence enhancement upon G-quadruplex folding: Synthesis, structure, and 
biophysical characterization of a dansyl/cyclodextrin-tagged thrombin binding aptamer," Bioconjugate Chemistry, vol. 24, no. 11, pp. 1917-1927, 2013.

[59] G. Oliviero, N. Borbone, J. Amato et al., "Synthesis of quadruplex-forming tetra-end-linked oligonucleotides: Effects of the linker size on quadruplex topology and stability," Biopolymers, vol. 91, no. 6, pp. 466-477, 2009.

[60] Z. Wang, X. Pei, N. Li, and X. Tang, "Phosphate-perylene modified G-quadruplex probes for the detection of $\mathrm{Pb} 2+$ using fluorescence anisotropy," Journal of Materials Chemistry B, vol. 4, no. 24, pp. 4330-4336, 2016.

[61] A. Risitano and K. R. Fox, "Influence of loop size on the stability of intramolecular DNA quadruplexes," Nucleic Acids Research, vol. 32, no. 8, pp. 2598-2606, 2004.

[62] S. S. Tavale and H. M. Sobell, "Crystal and molecular structure of 8-bromoguanosine and 8-bromoadenosine, two purine nucleosides in the syn conformation," Journal of Molecular Biology, vol. 48, no. 1, pp. 109-123, 1970.

[63] S. Uesugi and M. Ikehara, "Carbon-13 magnetic resonance spectra of 8-substituted purine nucleosides. Characteristic shifts for the syn conformation," Journal of the American Chemical Society, vol. 99, no. 10, pp. 3250-3253, 1977.

[64] E. Dias, J. L. Battiste, and J. R. Williamson, "Chemical probe for glycosidic conformation in telomeric DNAs," Journal of the American Chemical Society, vol. 116, no. 10, pp. 4479-4480, 1994.

[65] Y. Xu, Y. Noguchi, and H. Sugiyama, "The new models of the human telomere d[AGGG(TTAGGG)3] in K+ solution," Bioorganic and Medicinal Chemistry, vol. 14, no. 16, pp. 5584-5591, 2006.

[66] A. T. Phan and D. J. Patel, "Two-Repeat Human Telomeric d(TAGGGTTAGGGT) Sequence Forms Interconverting Parallel and Antiparallel G-Quadruplexes in Solution: Distinct Topologies, Thermodynamic Properties, and Folding/Unfolding Kinetics," Journal of the American Chemical Society, vol. 125, no. 49, pp. 15021-15027, 2003.

[67] V. Esposito, A. Randazzo, G. Piccialli, L. Petraccone, C. Giancola, and L. Mayol, "Effects of an 8-bromodeoxyguanosine incorporation on the parallel quadruplex structure [d(TGGGT)]4," Organic and Biomolecular Chemistry, vol. 2, no. 3, pp. 313-318, 2004.

[68] A. Matsugami, H. Tsuchibayashi, Y. Xu, Y. Noguchi, H. Sugiyama, and M. Katahira, "The new models of the human telomere DNA in $\mathrm{K}+$ solution revealed by NMR analysis assisted by the incorporation of 8-bromoguanines," Nucleic Acids Symposium Series (2004), no. 50, pp. 45-46, 2006.

[69] A. Matsugami, Y. Xu, Y. Noguchi, H. Sugiyama, and M. Katahira, "Structure of a human telomeric DNA sequence stabilized by 8-bromoguanosine substitutions, as determined by NMR in a K+ solution," FEBS Journal, vol. 274, no. 14, pp. 35453556, 2007.

[70] L. Petraccone, I. Duro, A. Randazzo, A. Virno, L. Mayol, and C. Giancola, "Biophysical properties of quadruplexes containing two or three 8-bromodeoxyguanosine residues," Nucleosides, Nucleotides and Nucleic Acids, vol. 26, no. 6-7, pp. 669-674, 2007.

[71] A. T. Phan, V. Kuryavyi, K. N. Luu, and D. J. Patel, "Structure of two intramolecular G-quadruplexes formed by natural human telomere sequences in K+ solution," Nucleic Acids Research, vol. 35, no. 19, pp. 6517-6525, 2007.

[72] T. Mashimo, Y. Sannohe, H. Yagi, and H. Sugiyama, "Folding pathways of hybrid-1 and hybrid-2 G-quadruplex structures," Nucleic acids symposium series (2004), no. 52, pp. 409-410, 2008.
[73] J. Gros, A. Aviñó, J. Lopez De La Osa et al., "8-Amino guanine accelerates tetramolecular G-quadruplex formation," Chemical Communications, no. 25, pp. 2926-2928, 2008.

[74] K. Okamoto, Y. Sannohe, T. Mashimo, H. Sugiyama, and M. Terazima, "G-quadruplex structures of human telomere DNA examined by single molecule FRET and BrG-substitution," Bioorganic and Medicinal Chemistry, vol. 16, no. 14, pp. 68736879, 2008.

[75] Y. Sannohe, K. Sato, A. Matsugami et al., "Orientation of ends of G-quadruplex structure investigated with end-extended oligonucleotides," Nucleic Acids Symposium Series (2004), no. 52, pp. 171-172, 2008.

[76] Y. Xu, H. Sato, Y. Sannohe, K.-I. Shinohara, and H. Sugiyama, "Stable lariat formation based on a G-quadruplex scaffold," Journal of the American Chemical Society, vol. 130, no. 49, pp. 16470-16471, 2008.

[77] K. W. Lim, S. Amrane, S. Bouaziz et al., "Structure of the human telomere in $\mathrm{K}+$ solution: A stable basket-type G-quadruplex with only two G-tetrad layers," Journal of the American Chemical Society, vol. 131, no. 12, pp. 4301-4309, 2009.

[78] Y. Xu, Y. Suzuki, T. Lönnberg, and M. Komiyama, "Human telomeric DNA sequence-specific cleaving by G-quadruplex formation," Journal of the American Chemical Society, vol. 131, no. 8, pp. 2871-2874, 2009.

[79] G. Lin, J. Zhang, Y. Zeng, H. Luo, and Y. Wang, "Conformationdependent formation of the G[8-5]U intrastrand cross-link in 5bromouracil-containing G-quadruplex DNA induced by UVA irradiation," Biochemistry, vol. 49, no. 11, pp. 2346-2350, 2010.

[80] C. J. Lech, J. K. Cheow Lim, J. M. Wen Lim, S. Amrane, B. Heddi, and A. T. Phan, "Effects of site-specific guanine c8modifications on an intramolecular DNA G-quadruplex," Biophysical Journal, vol. 101, no. 8, pp. 1987-1998, 2011.

[81] D. J. E. Yue, K. W. Lim, and A. T. Phan, "Formation of $(3+1)$ G-quadruplexes with a long loop by human telomeric DNA spanning five or more repeats," Journal of the American Chemical Society, vol. 133, no. 30, pp. 11462-11465, 2011.

[82] S. Goji and J. Matsui, "Direct detection of thrombin binding to 8-bromodeoxyguanosine-modified aptamer: Effects of modification on affinity and kinetics," Journal of Nucleic Acids, vol. 2011, Article ID 316079, 2011.

[83] A. I. Karsisiotis and M. W. Da Silva, "Structural probes in quadruplex nucleic acid structure determination by NMR," Molecules, vol. 17, no. 11, pp. 13073-13086, 2012.

[84] R. D. Gray, R. Buscaglia, and J. B. Chaires, "Populated intermediates in the thermal unfolding of the human telomeric quadruplex," Journal of the American Chemical Society, vol. 134, no. 40, pp. 16834-16844, 2012.

[85] A. T. Phan and N. Q. Do, "Engineering of interlocked DNA Gquadruplexes as a robust scaffold," Nucleic Acids Research, vol. 41, no. 4, pp. 2683-2688, 2013.

[86] K. W. Lim, V. C. M. Ng, N. Martín-Pintado, B. Heddi, and A. T. Phan, "Structure of the human telomere in $\mathrm{Na}+$ solution: An antiparallel $(2+2)$ G-quadruplex scaffold reveals additional diversity," Nucleic Acids Research, vol. 41, no. 22, pp. 1055610562, 2013.

[87] N. An, A. M. Fleming, and C. J. Burrows, "Interactions of the human telomere sequence with the nanocavity of the $\alpha$ hemolysin ion channel reveal structure-dependent electrical signatures for hybrid folds," Journal of the American Chemical Society, vol. 135, no. 23, pp. 8562-8570, 2013. 
[88] W. Gai, Q. Yang, J. Xiang et al., "A dual-site simultaneous binding mode in the interaction between parallel-stranded Gquadruplex [d(TGGGGT)]4 and cyanine dye 2,20-diethyl-9methylselenacarbocyanine bromide," Nucleic Acids Research, vol. 41, no. 4, pp. 2709-2722, 2013.

[89] J. Brčić and J. Plavec, "Solution structure of a DNA quadruplex containing ALS and FTD related GGGGCC repeat stabilized by 8-bromodeoxyguanosine substitution," Nucleic Acids Research, vol. 43, no. 17, pp. 8590-8600, 2015.

[90] M. Durec, F. Zaccaria, C. Fonseca Guerra, and R. Marek, "Modified Guanines as Constituents of Smart Ligands for Nucleic Acid Quadruplexes," Chemistry - A European Journal, vol. 22, no. 31, pp. 10912-10922, 2016.

[91] D. Gudanis, L. Popenda, K. Szpotkowski, R. Kierzek, and Z. Gdaniec, "Structural characterization of a dimer of RNA duplexes composed of 8-bromoguanosine modified CGG trinucleotide repeats: A novel architecture of RNA quadruplexes," Nucleic Acids Research, vol. 44, no. 5, pp. 2409-2416, 2016.

[92] B. Karg, L. Haase, A. Funke, J. Dickerhoff, and K. Weisz, "Observation of a Dynamic G-Tetrad Flip in Intramolecular GQuadruplexes," Biochemistry, vol. 55, no. 49, pp. 6949-6955, 2016.

[93] J. Brčić and J. Plavec, "ALS and FTD linked GGGGCC-repeat containing DNA oligonucleotide folds into two distinct Gquadruplexes," Biochimica et Biophysica Acta-General Subjects, 2016.

[94] A. Aviñó, S. Mazzini, C. Fàbrega et al., "The effect of 1thymidine, acyclic thymine and 8-bromoguanine on the stability of model G-quadruplex structures," Biochimica et Biophysica Acta-General Subjects, 2016.

[95] Y. Xu and H. Sugiyama, "Formation of the G-quadruplex and i-motif structures in retinoblastoma susceptibility genes (Rb)," Nucleic Acids Research, vol. 34, no. 3, pp. 949-954, 2006.

[96] A. Virgilio, V. Esposito, A. Randazzo, L. Mayol, and A. Galeone, "8-Methyl-2/-deoxyguanosine incorporation into parallel DNA quadruplex structures," Nucleic Acids Research, vol. 33, no. 19, pp. 6188-6195, 2005.

[97] A. Virgilio, V. Esposito, G. Citarella, A. Pepe, L. Mayol, and A. Galeone, "The insertion of two 8-methyl-2I-deoxyguanosine residues in tetramolecular quadruplex structures: Trying to orientate the strands," Nucleic Acids Research, vol. 40, no. 1, pp. 461-475, 2012.

[98] P. L. T. Tran, A. Virgilio, V. Esposito, G. Citarella, J.-L. Mergny, and A. Galeone, "Effects of 8-methylguanine on structure, stability and kinetics of formation of tetramolecular quadruplexes," Biochimie, vol. 93, no. 3, pp. 399-408, 2011.

[99] A. Virgilio, V. Esposito, G. Citarella, L. Mayol, and A. Galeone, "Structural Investigations on the Anti-HIV G-QuadruplexForming Oligonucleotide TGGGAG and Its Analogues: Evidence for the Presence of an A-Tetrad," ChemBioChem, vol. 13, no. 15, pp. 2219-2224, 2012.

[100] Z. Zhao, F. Lin, H. Ye, R. Huang, and X. Xu, "Effects of modifiedguanosine on the stability of G-triplex," Tetrahedron Letters, vol. 57, no. 48, pp. 5321-5325, 2016.

[101] K. N. Luu, A. T. Phan, V. Kuryavyi, L. Lacroix, and D. J. Patel, "Structure of the human telomere in $\mathrm{K}+$ solution: An intramolecular $(3+1)$ G-quadruplex scaffold," Journal of the American Chemical Society, vol. 128, no. 30, pp. 9963-9970, 2006.

[102] J. L. De La Osa, C. González, R. Gargallo et al., "Destabilization of quadruplex DNA by 8-aminoguanine," ChemBioChem, vol. 7, no. 1, pp. 46-48, 2006.
[103] R. Ferreira, M. Alvira, A. Aviñó et al., "Synthesis and structural characterization of stable branched DNA G-quadruplexes using the trebler phosphoramidite," ChemistryOpen, vol. 1, no. 2, pp. 106-114, 2012.

[104] J. Sági, A. Szemzö, K. Ébinger et al., "Base-modified oligodeoxynucleotides. I. effect of 5-alkyl, 5-(1-alkenyl) and 5-(1alkynyl) substitution of the pyrimidines on duplex stability and hydrophobicity," Tetrahedron Letters, vol. 34, no. 13, pp. 21912194, 1993.

[105] A. Nadler, J. Strohmeier, and U. Diederichsen, "8-vinyl-2/deoxyguanosine as a fluorescent $2 /$-deoxyguanosine mimic for investigating DNA hybridization and topology," Angewandte Chemie - International Edition, vol. 50, no. 23, pp. 5392-5396, 2011.

[106] S. Müllar, J. Strohmeier, and U. Diederichsen, "8-vinylguanine nucleo amino acid: A fluorescent PNA building block," Organic Letters, vol. 14, no. 6, pp. 1382-1385, 2012.

[107] A. Dumas and N. W. Luedtke, "Highly fluorescent guanosine mimics for folding and energy transfer studies," Nucleic Acids Research, vol. 39, no. 15, pp. 6825-6834, 2011.

[108] S. Ogasawara and M. Maeda, "Photo-controllable aptamer," Nucleic Acids Symposium Series, vol. 53, no. 1, pp. 195-196, 2009.

[109] A. Dumas and N. W. Luedtke, "Cation-mediated energy transfer in G-quadruplexes revealed by an internal fluorescent probe," Journal of the American Chemical Society, vol. 132, no. 51, pp. 18004-18007, 2010.

[110] M. Sproviero, K. L. Fadock, A. A. Witham, R. A. Manderville, P. Sharma, and S. D. Wetmore, "Electronic tuning of fluorescent 8 -aryl-guanine probes for monitoring DNA duplex-quadruplex exchange," Chemical Science, vol. 5, no. 2, pp. 788-796, 2014.

[111] M. Sproviero and R. A. Manderville, "Harnessing G-tetrad scaffolds within G-quadruplex forming aptamers for fluorescence detection strategies," Chemical Communications, vol. 50, no. 23, pp. 3097-3099, 2014.

[112] D. J. M. Blanchard, T. Z. Cservenyi, and R. A. Manderville, "Dual fluorescent deoxyguanosine mimics for FRET detection of G-quadruplex folding," Chemical Communications, vol. 51, no. 94, pp. 16829-16831, 2015.

[113] D. J. M. Blanchard, K. L. Fadock, M. Sproviero et al., "Photophysical properties of push-pull 8-aryl-deoxyguanosine probes within duplex and G-quadruplex structures," Journal of Materials Chemistry C, vol. 4, no. 14, pp. 2915-2924, 2016.

[114] K. L. Fadock, R. A. Manderville, P. Sharma, and S. D. Wetmore, "Optimization of fluorescent 8-heteroaryl-guanine probes for monitoring protein-mediated duplex $\rightarrow$ G-quadruplex exchange," Organic and Biomolecular Chemistry, vol. 14, no. 19, pp. 4409-4419, 2016.

[115] R. A. Manderville and S. D. Wetmore, "C-Linked 8-Aryl guanine nucleobase adducts: Biological outcomes and utility as fluorescent probes," Chemical Science, vol. 7, no. 6, pp. 34823493, 2016.

[116] M. Sproviero, K. L. Fadock, A. A. Witham, and R. A. Manderville, "Positional Impact of Fluorescently Modified GTetrads within Polymorphic Human Telomeric G-Quadruplex Structures," ACS Chemical Biology, vol. 10, no. 5, pp. 1311-1318, 2015.

[117] D. J. M. Blanchard and R. A. Manderville, "An internal charge transfer-DNA platform for fluorescence sensing of divalent metal ions," Chemical Communications, vol. 52, no. 61, pp. 95869588, 2016. 
[118] A. Dumas and N. W. Luedtke, "Site-specific control of N7metal coordination in DNA by a fluorescent purine derivative," Chemistry - A European Journal, vol. 18, no. 1, pp. 245-254, 2012.

[119] J. Li, J. J. Correia, L. Wang, J. O. Trent, and J. B. Chaires, "Not so crystal clear: the structure of the human telomere G-quadruplex in solution differs from that present in a crystal," Nucleic Acids Research, vol. 33, no. 14, pp. 4649-4659, 2005.

[120] R. D. Gray, L. Petraccone, J. O. Trent, and J. B. Chaires, "Characterization of a $\mathrm{K}+$-induced conformational switch in a human telomeric DNA oligonucleotide using 2-aminopurine fluorescence," Biochemistry, vol. 49, no. 1, pp. 179-194, 2010.

[121] R. Buscaglia, D. M. Jameson, and J. B. Chaires, "G-quadruplex structure and stability illuminated by 2 -aminopurine phasor plots," Nucleic Acids Research, vol. 40, no. 9, pp. 4203-4215, 2012.

[122] T. Kimura, K. Kawai, M. Fujitsuka, and T. Majima, "Monitoring G-quadruplex structures and G-quadruplex-ligand complex using 2-aminopurine modified oligonucleotides," Tetrahedron, vol. 63, no. 17, pp. 3585-3590, 2007.

[123] C. Y. Lee, K. S. Park, and H. G. Park, "A fluorescent Gquadruplex probe for the assay of base excision repair enzyme activity," Chemical Communications, vol. 51, no. 72, pp. 1374413747, 2015.

[124] J. Johnson, R. Okyere, A. Joseph, K. Musier-Forsyth, and B. Kankia, "Quadruplex formation as a molecular switch to turn on intrinsically fluorescent nucleotide analogs," Nucleic Acids Research, vol. 41, no. 1, pp. 220-228, 2013.

[125] C. M. Olsen, H.-T. Lee, and L. A. Marky, "Unfolding thermodynamics of intramolecular G-quadruplexes: Base sequence contributions of the loops," Journal of Physical Chemistry B, vol. 113, no. 9, pp. 2587-2595, 2009.

[126] V. B. Tsvetkov, A. M. Varizhuk, G. E. Pozmogova, I. P. Smirnov, N. A. Kolganova, and E. N. Timofeev, "A Universal Base in a Specific Role: Tuning up a Thrombin Aptamer with 5Nitroindole," Scientific Reports, vol. 5, Article ID 16337, 2015.

[127] I. Smirnov and R. H. Shafer, "Effect of loop sequence and size on DNA aptamer stability," Biochemistry, vol. 39, no. 6, pp. 14621468, 2000.

[128] P. Hazel, J. Huppert, S. Balasubramanian, and S. Neidle, "Looplength-dependent folding of G-quadruplexes," Journal of the American Chemical Society, vol. 126, no. 50, pp. 16405-16415, 2004.

[129] P. A. Rachwal, T. Brown, and K. R. Fox, "Sequence effects of single base loops in intramolecular quadruplex DNA," FEBS Letters, vol. 581, no. 8, pp. 1657-1660, 2007.

[130] P. A. Rachwal, I. S. Findlow, J. M. Werner, T. Brown, and K. R. Fox, "Intramolecular DNA quadruplexes with different arrangements of short and long loops," Nucleic Acids Research, vol. 35, no. 12, pp. 4214-4222, 2007.

[131] T. Z. Cservenyi, A. J. Van Riesen, F. D. Berger, A. Desoky, and R. A. Manderville, "A Simple Molecular Rotor for Defining Nucleoside Environment within a DNA Aptamer-Protein Complex," ACS Chemical Biology, vol. 11, no. 9, pp. 2576-2582, 2016.

[132] Y. Xu and H. Sugiyama, "Highly efficient photochemical 2ıdeoxyribonolactone formation at the diagonal loop of a 5iodouracil-containing antiparallel G-quartet," Journal of the American Chemical Society, vol. 126, no. 20, pp. 6274-6279, 2004.

[133] C. Cheong and P. B. Moore, "Solution structure of an unusually stable RNA tetraplex containing G- and U-quartet structures," Biochemistry, vol. 31, no. 36, pp. 8406-8414, 1992.
[134] J. Deng, Y. Xiong, and M. Sundaralingam, "X-ray analysis of an RNA tetraplex (UGGGGU)4 with divalent Sr2+ ions at subatomic resolution (0.61 $\AA$ )," Proceedings of the National Academy of Sciences of the United States of America, vol. 98, no. 24, pp. 13665-13670, 2001.

[135] B. Pan, Y. Xiong, K. Shi, J. Deng, and M. Sundaralingam, "Crystal structure of an RNA purine-rich tetraplex containing adenine tetrads: Implications for specific binding in RNA tetraplexes," Structure, vol. 11, no. 7, pp. 815-823, 2003.

[136] C.-F. Tang and R. H. Shafer, "Engineering the quadruplex fold: Nucleoside conformation determines both folding topology and molecularity in guanine quadruplexes," Journal of the American Chemical Society, vol. 128, no. 17, pp. 5966-5973, 2006.

[137] P. Schultze, N. V. Hud, F. W. Smith, and J. Feigon, “The effect of sodium, potassium and ammonium ions on the conformation of the dimeric quadruplex formed by the Oxytricha nova telomere repeat oligonucleotide d(G4T4G4)," Nucleic Acids Research, vol. 27, no. 15, pp. 3018-3028, 1999.

[138] J. Vondrušková, J. Kypr, I. Kejnovská, M. Fialová, and M. Vorlícková, "Guanine quadruplex formation by RNA/DNA hybrid analogs of Oxytricha telomere G4T4G4 fragment," Biopolymers, vol. 89, no. 10, pp. 797-806, 2008.

[139] J. Vondrušková, J. Kypr, I. Kejnovská, M. Fialová, and M. Vorlíčková, "Role of loops in the guanine quadruplex formation by DNA/RNA hybrid analogs of G4T4G4," International Journal of Biological Macromolecules, vol. 43, no. 5, pp. 463-467, 2008.

[140] J. Dickerhoff, B. Appel, S. Müller, and K. Weisz, "Sugar-Edge Interactions in a DNA-RNA G-Quadruplex: Evidence of Sequential C-H. . O Hydrogen Bonds Contributing to RNA Quadruplex Folding," Angewandte Chemie - International Edition, vol. 55, no. 48, pp. 15162-15165, 2016.

[141] J. Zhou, S. Amrane, F. Rosu et al., "Unexpected PositionDependent Effects of Ribose G-Quartets in G-Quadruplexes," Journal of the American Chemical Society, vol. 139, no. 23, pp. 7768-7779, 2017

[142] L. Zhu, C. Li, Z. Zhu et al., "In vitro selection of highly efficient G-quadruplex-based DNAzymes," Analytical Chemistry, vol. 84, no. 19, pp. 8383-8390, 2012.

[143] A. Aviñó, S. Mazzini, R. Ferreira, R. Gargallo, V. E. Marquez, and R. Eritja, "The effect on quadruplex stability of Northnucleoside derivatives in the loops of the thrombin-binding aptamer," Bioorganic and Medicinal Chemistry, vol. 20, no. 14, pp. 4186-4193, 2012.

[144] C. G. Peng and M. J. Damha, "G-quadruplex induced stabilization by $2 /$-deoxy-2/-fluoro-d-arabinonucleic acids (2/F-ANA)," Nucleic Acids Research, vol. 35, no. 15, pp. 4977-4988, 2007.

[145] C. J. Lech, Z. Li, B. Heddi, and A. T. Phan, "2l-F-ANAguanosine and $2 /$-F-guanosine as powerful tools for structural manipulation of G-quadruplexes," Chemical Communications, vol. 48, no. 93, pp. 11425-11427, 2012.

[146] A. T. Phan, K. N. Luu, and D. J. Patel, "Different loop arrangements of intramolecular human telomeric (3+1) Gquadruplexes in K+ solution," Nucleic Acids Research, vol. 34, no. 19, pp. 5715-5719, 2006.

[147] N. Martín-Pintado, M. Yahyaee-Anzahaee, G. F. Deleavey et al., "Dramatic effect of furanose C21 substitution on structure and stability: Directing the folding of the human telomeric quadruplex with a single fluorine atom," Journal of the American Chemical Society, vol. 135, no. 14, pp. 5344-5347, 2013. 
[148] Z. Liy, C. J. Lechy, and A. T. Phan, "Sugar-modified Gquadruplexes: Effects of LNA-, 20F-RNA-and 20F-ANA-guanosine chemistries on G-quadruplex structure and stability," Nucleic Acids Research, vol. 42, no. 6, pp. 4068-4079, 2014.

[149] J. Dickerhoff and K. Weisz, "Flipping a G-tetrad in a unimolecular quadruplex without affecting its global fold," Angewandte Chemie - International Edition, vol. 54, no. 19, pp. 5588-5591, 2015.

[150] J. Dickerhoff, L. Haase, W. Langel, and K. Weisz, “Tracing Effects of Fluorine Substitutions on G-Quadruplex Conformational Changes," ACS Chemical Biology, vol. 12, no. 5, pp. 1308-1315, 2017.

[151] J. Lietard, H. Abou Assi, I. Gómez-Pinto, C. González, M. M. Somoza, and M. J. Damha, "Mapping the affinity landscape of Thrombin-binding aptamers on 2/F-ANA/DNA chimeric GQuadruplex microarrays," Nucleic Acids Research, p. gkw1357.

[152] M. Petersen and J. Wengel, "LNA: a versatile tool for therapeutics and genomics," Trends in Biotechnology, vol. 21, no. 2, pp. 74-81, 2003.

[153] M. Petersen, K. Bondensgaard, J. Wengel, and J. Peter Jacobsen, "Locked nucleic acid (LNA) recognition of RNA: NMR solution structures of LNA:RNA hybrids," Journal of the American Chemical Society, vol. 124, no. 21, pp. 5974-5982, 2002.

[154] A. Randazzo, V. Esposito, O. Ohlenschläger, R. Ramachandran, and L. Mayol, "NMR solution structure of a parallel LNA quadruplex," Nucleic Acids Research, vol. 32, no. 10, pp. 30833092, 2004.

[155] J. T. Nielsen, K. Arar, and M. Petersen, "NMR solution structures of LNA (locked nucleic acid) modified quadruplexes," Nucleic Acids Research, vol. 34, no. 7, pp. 2006-2014, 2006.

[156] J. T. Nielsen, K. Arar, and M. Petersen, "Solution structure of a locked nucleic acid modified quadruplex: Introducing the V4 folding topology," Angewandte Chemie - International Edition, vol. 48, no. 17, pp. 3099-3103, 2009.

[157] P. Schultze, F. W. Smith, and J. Feigon, "Refined solution structure of the dimeric quadruplex formed from the Oxytricha telomeric oligonucleotide d(GGGGTTTTGGGG)," Structure, vol. 2, no. 3, pp. 221-233, 1994.

[158] S. Haider, G. N. Parkinson, and S. Neidle, "Crystal structure of the potassium form of an Oxytricha nova G-quadruplex," Journal of Molecular Biology, vol. 320, no. 2, pp. 189-200, 2002.

[159] N. Zhang, A. Gorin, A. Majumdar et al., "V-shaped scaffold: A new architectural motif identified in an A.(G.G.G.G) pentad-containing dimeric DNA quadruplex involving stacked G(anti).G(anti).G(anti).G(syn) tetrads," Journal of Molecular Biology, vol. 311, no. 5, pp. 1063-1079, 2001.

[160] P. K. Dominick and M. B. Jarstfer, "A Conformationally Constrained Nucleotide Analogue Controls the Folding Topology of a DNA G-Quadruplex," Journal of the American Chemical Society, vol. 126, no. 16, pp. 5050-5051, 2004.

[161] A. Virno, A. Randazzo, C. Giancola, M. Bucci, G. Cirino, and L. Mayol, "A novel thrombin binding aptamer containing a GLNA residue," Bioorganic and Medicinal Chemistry, vol. 15, no. 17, pp. 5710-5718, 2007.

[162] L. Bonifacio, F. C. Church, and M. B. Jarstfer, "Effect of lockednucleic acid on a biologically active G-quadruplex. A structureactivity relationship of the thrombin aptamer," International Journal of Molecular Sciences, vol. 9, no. 3, pp. 422-433, 2008.

[163] D. Pradhan, L. H. Hansen, B. Vester, and M. Petersen, "Selection of G-quadruplex folding topology with LNA-modified human telomeric sequences in $\mathrm{K}+$ solution," Chemistry - A European Journal, vol. 17, no. 8, pp. 2405-2413, 2011.
[164] K. I. Russo, G. N. Parkinson, A. Merlino et al., "A regular thymine tetrad and a peculiar supramolecular assembly in the first crystal structure of an all-LNA G-quadruplex," Acta Crystallographica Section D: Biological Crystallography, vol. 70, no. 2, pp. 362-370, 2014.

[165] O. Gissberg, E. M. Zaghloul, K. E. Lundin et al., "Delivery, Effect on Cell Viability, and Plasticity of Modified Aptamer Constructs," Nucleic Acid Therapeutics, vol. 26, no. 3, pp. 183189, 2016.

[166] M. Marušič, R. N. Veedu, J. Wengel, and J. Plavec, "G-rich VEGF aptamer with locked and unlocked nucleic acid modifications exhibits a unique G-quadruplex fold," Nucleic Acids Research, vol. 41, no. 20, pp. 9524-9536, 2013.

[167] Y. Kasahara, S. Kitadume, K. Morihiro et al., "Effect of 3/end capping of aptamer with various $2 /, 4 /$-bridged nucleotides: Enzymatic post-modification toward a practical use of polyclonal aptamers," Bioorganic and Medicinal Chemistry Letters, vol. 20, no. 5, pp. 1626-1629, 2010.

[168] E. B. Pedersen, J. T. Nielsen, C. Nielsen, and V. V. Filichev, "Enhanced anti-HIV-1 activity of G-quadruplexes comprising locked nucleic acids and intercalating nucleic acids," Nucleic Acids Research, vol. 39, no. 6, pp. 2470-2481, 2011.

[169] K. Takahama and T. Oyoshi, "Specific binding of modified rgg domain in tls/fus to g-quadruplex rna: Tyrosines in rgg domain recognize 2I-oh of the riboses of loops in g-quadruplex," Journal of the American Chemical Society, vol. 135, no. 48, pp. 1801618019, 2013.

[170] A. Pasternak, F. J. Hernandez, L. M. Rasmussen, B. Vester, and J. Wengel, "Improved thrombin binding aptamer by incorporation of a single unlocked nucleic acid monomer," Nucleic Acids Research, vol. 39, no. 3, pp. 1155-1164, 2011.

[171] T. B. Jensen, J. R. Henriksen, B. E. Rasmussen et al., "Thermodynamic and biological evaluation of a thrombin binding aptamer modified with several unlocked nucleic acid (UNA) monomers and a 21 -C-piperazino-UNA monomer," Bioorganic and Medicinal Chemistry, vol. 19, no. 16, pp. 4739-4745, 2011.

[172] W. Kotkowiak, R. Kierzek, and A. Pasternak, "Thermodynamic, structural and biological features of thrombin binding aptamer modified with novel UNA derivative," in Proceedings of theMeeting Abstracts, 5th International Meeting on Quadruplex Nucleic Acids: G4thering in Bordeaux, Bordeaux, France, May 26-28, 2015.

[173] T. Agarwal, S. Kumar, and S. Maiti, "Unlocking G-quadruplex: Effect of unlocked nucleic acid on G-quadruplex stability," Biochimie, vol. 93, no. 10, pp. 1694-1700, 2011.

[174] F. Morvan, F. Debart, and J.-J. Vasseur, "From anionic to cationic $\alpha$-anomeric oligodeoxynucleotides," Chemistry and Biodiversity, vol. 7, no. 3, pp. 494-535, 2010.

[175] D. J. Ecker, T. A. Vickers, R. Hanecak, V. Diver, and K. Anderson, "Rational screening of oligonucleotide combinatorial libraries for drug discovery," Nucleic Acids Research, vol. 21, no. 8, pp. 1853-1856, 1993.

[176] J. R. Wyatt, T. A. Vickers, J. L. Roberson et al., "Combinatorially selected guanosine-quartet structure is a potent inhibitor of human immunodeficiency virus envelope-mediated cell fusion," Proceedings of the National Academy of Sciences of the United States of America, vol. 91, no. 4, pp. 1356-1360, 1994.

[177] Y. S. Latha and N. Yathindra, "Stereochemical studies on nucleic acid analogues. I. Conformations of $\alpha$-nucleosides and $\alpha$ nucleotides: Interconversion of sugar puckers via O4/-exo," Biopolymers, vol. 32, no. 3, pp. 249-269, 1992. 
[178] N. A. Kolganova, A. M. Varizhuk, R. A. Novikov et al., "Anomeric DNA quadruplexes," Artificial DNA, PNA \& XNA, vol. 5, no. 2, p. e28422, 2014.

[179] H. Urata, T. Kumashiro, T. Otake, T. Kawahata, and M. Akagi, "Anti-HIV-1 activity of L-DNA quadruplex," Nucleic Acids Research, supplement (2001), no. 2, pp. 163-164, 2002.

[180] P. L. T. Tran, R. Moriyama, A. Maruyama, B. Rayner, and J.L. Mergny, "A mirror-image tetramolecular DNA quadruplex," Chemical Communications, vol. 47, no. 19, pp. 5437-5439, 2011.

[181] A. Virgilio, V. Esposito, G. Citarella, A. Mangoni, L. Mayol, and A. Galeone, "Unprecedented right- and left-handed quadruplex structures formed by heterochiral oligodeoxyribonucleotides," Biochimie, vol. 93, no. 7, pp. 1193-1196, 2011.

[182] A. Virgilio, M. Varra, M. Scuotto et al., "Expanding the potential of G-quadruplex structures: formation of a heterochiral TBA analogue," ChemBioChem, vol. 15, no. 5, pp. 652-655, 2014.

[183] A. Virgilio, V. Esposito, A. Mangoni, L. Mayol, and A. Galeone, "A novel equilibrium relating to the helix handedness in Gquadruplexes formed by heterochiral oligonucleotides with an inversion of polarity site," Chemical Communications, vol. 49, no. 72, pp. 7935-7937, 2013.

[184] A. Virgilio, L. Mayol, M. Varra, A. Galeone, and V. Esposito, "Unusual chair-like g-quadruplex structures: Heterochiral tba analogues containing inversion of polarity sites," Journal of Chemistry, vol. 2015, Article ID 473051, 2015.

[185] M. Szabat, D. Gudanis, W. Kotkowiak, Z. Gdaniec, R. Kierzek, and A. Pasternak, "Thermodynamic features of structural motifs formed by $\beta$-L-RNA," PLoS ONE, vol. 11, no. 2 , Article ID e0149478, 2016.

[186] L. Yatime, C. Maasch, K. Hoehlig, S. Klussmann, G. R. Andersen, and A. Vater, "Structural basis for the targeting of complement anaphylatoxin C5a using a mixed L-RNA/L-DNA aptamer," Nature Communications, vol. 6, article 6481, 2015.

[187] L. Yuan, T. Tian, Y. Chen, Z. Zhang, and X. Zhou, "An LDNA G-Quadruplex: Application for peroxidase DNAzyme," Nucleosides, Nucleotides and Nucleic Acids, vol. 32, no. 11, pp. 589-598, 2013.

[188] B. Cai, X. Yang, L. Sun et al., "Stability and bioactivity of thrombin binding aptamers modified with," Organic \& Biomolecular Chemistry, vol. 12, no. 44, pp. 8866-8876, 2014.

[189] H. Saneyoshi, S. Mazzini, A. Aviñó et al., “The use of conformationally rigid nucleoside probes to study the role of sugar pucker and nucleobase orientation in the thrombin binding aptamer," Nucleic Acids Symposium Series (2004), no. 53, pp. 109-110, 2009.

[190] H. Saneyoshi, S. Mazzini, A. Aviñó et al., "Conformationally rigid nucleoside probes help understand the role of sugar pucker and nucleobase orientation in the thrombin-binding aptamer," Nucleic Acids Research, vol. 37, no. 17, Article ID gkp598, pp. 5589-5601, 2009.

[191] T. Coppola, M. Varra, G. Oliviero et al., "Synthesis, structural studies and biological properties of new TBA analogues containing an acyclic nucleotide," Bioorganic and Medicinal Chemistry, vol. 16, no. 17, pp. 8244-8253, 2008.

[192] C. Li, L. Zhu, Z. Zhu et al., "Backbone modification promotes peroxidase activity of G-quadruplex-based DNAzyme," Chemical Communications, vol. 48, no. 67, pp. 8347-8349, 2012.

[193] P. J. Bates, J. B. Kahlon, S. D. Thomas, J. O. Trent, and D. M. Miller, "Antiproliferative activity of G-rich oligonucleotides correlates with protein binding," The Journal of Biological Chemistry, vol. 274, no. 37, pp. 26369-26377, 1999.
[194] V. Dapić, P. J. Bates, J. O. Trent, A. Rodger, S. D. Thomas, and D. M. Miller, "Antiproliferative activity of G-quartet-forming oligonucleotides with backbone and sugar modifications," Biochemistry, vol. 41, no. 11, pp. 3676-3685, 2002.

[195] B. Liu and D. Li, "Structural transformation induced by locked nucleic acid or 2/-O-methyl nucleic acid site-specific modifications on thrombin binding aptamer," Chemistry Central Journal, vol. 8, no. 1, article no. 19, 2014.

[196] I. Gómez-Pinto, E. Vengut-Climent, R. Lucas et al., "Carbohydrate-DNA interactions at G-quadruplexes: Folding and stability changes by attaching sugars at the 5/-End," Chemistry A European Journal, vol. 19, no. 6, pp. 1920-1927, 2013.

[197] S. Grijalvo, A. Alagia, R. Gargallo, and R. Eritja, "Cellular uptake studies of antisense oligonucleotides using G-quadruplexnanostructures. the effect of cationic residue on the biophysical and biological properties," RSC Advances, vol. 6, no. 80, pp. 76099-76109, 2016.

[198] M. Boczkowska, P. Guga, and W. J. Stec, "Stereodefined phosphorothioate analogues of DNA: Relative thermodynamic stability of the model PS-DNA/DNA and PS-DNA/RNA complexes," Biochemistry, vol. 41, no. 41, pp. 12483-12487, 2002.

[199] F. Eckstein, "Phosphorothioates, essential components of therapeutic oligonucleotides," Nucleic Acid Therapeutics, vol. 24, no. 6, pp. 374-387, 2014.

[200] M. Zaitseva, D. Kaluzhny, A. Shchyolkina, O. Borisova, I. Smirnov, and G. Pozmogova, "Conformation and thermostability of oligonucleotide d(GGTTGGTGTGGTTGG) containing thiophosphoryl internucleotide bonds at different positions," Biophysical Chemistry, vol. 146, no. 1, pp. 1-6, 2010.

[201] X. Zhang, C.-X. Xu, R. Di Felice et al., "Conformations of Human Telomeric G-Quadruplex Studied Using a NucleotideIndependent Nitroxide Label," Biochemistry, vol. 55, no. 2, pp. 360-372, 2016.

[202] V. Esposito, A. Virgilio, A. Randazzo, A. Galeone, and L. Mayol, "A new class of DNA quadruplexes formed by oligodeoxyribonucleotides containing a $3^{\prime}-3^{\prime}$ or $5^{\prime}-5^{\prime}$ inversion of polarity site," Chemical Communications, no. 31, pp. 3953-3955, 2005.

[203] V. Esposito, A. Galeone, L. Mayol, A. Randazzo, A. Virgilio, and A. Virno, "A mini-library of TBA analogues containing 3 ' -3 ' and $5^{\prime}-5^{\prime}$ inversion of polarity sites," Nucleosides, Nucleotides and Nucleic Acids, vol. 26, no. 8-9, pp. 1145-1149, 2007.

[204] L. Martino, A. Virno, A. Randazzo et al., "A new modified thrombin binding aptamer containing a $5^{\prime}-5^{\prime}$ inversion of polarity site," Nucleic Acids Research, vol. 34, no. 22, pp. 66536662, 2006.

[205] B. Pagano, L. Martino, A. Randazzo, and C. Giancola, "Stability and binding properties of a modified thrombin binding aptamer," Biophysical Journal, vol. 94, no. 2, pp. 562-569, 2008.

[206] A. Galeone, L. Mayol, A. Virgilio, A. Virno, and A. Randazzo, "A further contribution to the extreme variability of quadruplex structures from oligodeoxyribonucleotides containing inversion of polarity sites in the G-tract," Molecular BioSystems, vol. 4, no. 5, pp. 426-430, 2008.

[207] A. Virno, F. Zaccaria, A. Virgilio et al., "Molecular modelling studies of four stranded quadruplexes containing a 3/-3/ or 5/-5/ inversion of polarity site," Nucleosides, Nucleotides and Nucleic Acids, vol. 26, no. 8-9, pp. 1139-1143, 2007.

[208] V. Esposito, A. Virgilio, A. Pepe, G. Oliviero, L. Mayol, and A. Galeone, "Effects of the introduction of inversion of polarity sites in the quadruplex forming oligonucleotide TGGGT," Bioorganic and Medicinal Chemistry, vol. 17, no. 5, pp. 1997-2001, 2009. 
[209] A. Virgilio, V. Esposito, L. Mayol, and A. Galeone, "More than one non-canonical phosphodiester bond in the G-tract: formation of unusual parallel G-quadruplex structures," Organic \& Biomolecular Chemistry, vol. 12, no. 3, pp. 534-540, 2014.

[210] V. Esposito, M. Scuotto, A. Capuozzo et al., "A straightforward modification in the thrombin binding aptamer improving the stability, affinity to thrombin and nuclease resistance," Organic \& Biomolecular Chemistry, vol. 12, no. 44, pp. 8840-8843, 2014.

[211] A. Virgilio, A. Russo, T. Amato et al., "Monomolecular Gquadruplex structures with inversion of polarity sites: new topologies and potentiality," Nucleic Acids Research, vol. 45, no. 14, pp. 8156-8166, 2017.

[212] G. Oliviero, S. D'Errico, B. Pinto et al., "Self-Assembly of G-Rich Oligonucleotides Incorporating a 3/-3/ Inversion of Polarity Site: A New Route Towards G-Wire DNA Nanostructures," ChemistryOpen, vol. 6, no. 4, pp. 599-605, 2017.

[213] P. Šket, A. Virgilio, V. Esposito, A. Galeone, and J. Plavec, "Strand directionality affects cation binding and movement within tetramolecular G-quadruplexes," Nucleic Acids Research, vol. 40, no. 21, pp. 11047-11057, 2012.

[214] M. Marušič and J. Plavec, "The Effect of DNA Sequence Directionality on G-Quadruplex Folding," Angewandte Chemie - International Edition, vol. 54, no. 40, pp. 11716-11719, 2015.

[215] V. Romanucci, M. Gaglione, A. Messere et al., "Hairpin oligonucleotides forming G-quadruplexes: New aptamers with antiHIV activity," European Journal of Medicinal Chemistry, vol. 89, pp. 51-58, 2014.

[216] V. Esposito, A. Russo, T. Amato et al., "Backbone modified TBA analogues endowed with antiproliferative activity," Biochimica et Biophysica Acta - General Subjects, 2016.

[217] V. Esposito, G. Oliviero, A. Pepe, A. Virgilio, and A. Galeone, "Studies on the influence of inversion of polarity sites on the dG residues glycosidic conformation in quadruplex structures," Nucleic Acids Symposium Series (2004), no. 52, pp. 177-178, 2008.

[218] A. S. Gouda, M. S. Amine, and E. B. Pedersen, "Synthesis of New DNA G-Quadruplex Constructs with Anthraquinone Insertions and Their Anticoagulant Activity," Helvetica Chimica Acta, vol. 99, no. 2, pp. 116-124, 2016.

[219] A. S. Gouda, M. S. Amine, and E. B. Pedersen, "Synthesis and Molecular Modeling of Thermally Stable DNA G-Quadruplexes with Anthraquinone Insertions," European Journal of Organic Chemistry, vol. 2017, no. 21, pp. 3092-3100, 2017.

[220] P. Li, J. Sun, M. Su, X. Yang, and X. Tang, "Design, synthesis and properties of artificial nucleic acids from (R)-4-amino-butane1,3-diol," Organic and Biomolecular Chemistry, vol. 12, no. 14, pp. 2263-2272, 2014.

[221] S. K. Rajagopal and M. Hariharan, "Non-natural G-quadruplex in a non-natural environment," Photochemical \& Photobiological Sciences, vol. 13, no. 2, pp. 157-161, 2014.

[222] J. Gros, F. Rosu, S. Amrane et al., "Guanines are a quartet's best friend: Impact of base substitutions on the kinetics and stability of tetramolecular quadruplexes," Nucleic Acids Research, vol. 35, no. 9, pp. 3064-3075, 2007.

[223] V. M. Marathias, M. J. Sawicki, and P. H. Bolton, "6-Thioguanine alters the structure and stability of duplex DNA and inhibits quadruplex DNA formation," Nucleic Acids Research, vol. 27, no. 14, pp. 2860-2867, 1999.

[224] F. W. Smith, P. Schultze, and J. Feigon, "Solution structures of unimolecular quadruplexes formed by oligonucleotides containing Oxytricha telomere repeats," Structure, vol. 3, no. 10, pp. 997-1008, 1995.
[225] N. Špačkova, E. Cubero, J. Šponer, and M. Orozco, “Theoretical study of the guanine $\rightarrow 6$-thioguanine substitution in duplexes, triplexes, and tetraplexes," Journal of the American Chemical Society, vol. 126, no. 44, pp. 14642-14650, 2004.

[226] R. Radhika, R. Shankar, S. Vijayakumar, and P. Kolandaivel, "Role of 6-Mercaptopurine in the potential therapeutic targets DNA base pairs and G-quadruplex DNA: insights from Quantum chemical and Molecular dynamics simulations," Journal of Biomolecular Structure and Dynamics, Article ID 1323013, pp. 1-33, 2017.

[227] I. Faustino, C. Curutchet, F. J. Luque, and M. Orozco, "The DNA-forming properties of 6-selenoguanine," Physical Chemistry Chemical Physics, vol. 16, no. 3, pp. 1101-1110, 2014.

[228] A. I. H. Murchie and D. M. J. Lilley, "Retinoblastoma susceptibility genes contain 5 ' sequences with a high propensity to form guanine-tetrad structures," Nucleic Acids Research, vol. 20, no. 1, pp. 49-53, 1992.

[229] L. Benimetskaya, M. Berton, A. Kolbanovsky, S. Benimetsky, and C. A. Stein, "Formation of a G-tetrad and higher order structures correlates with biological activity of the RelA (NF- $\kappa \mathrm{B}$ p65) 'antisense' oligodeoxynucleotide," Nucleic Acids Research, vol. 25, no. 13, pp. 2648-2656, 1997.

[230] B. Samatanga, C. Dominguez, I. Jelesarov, and F. H.-T. Allain, "The high kinetic stability of a G-quadruplex limits hnRNP F qRRM3 binding to G-tract RNA," Nucleic Acids Research, vol. 41, no. 4, pp. 2505-2516, 2013.

[231] F. Römmler, M. Hammel, A. Waldhuber et al., "Guaninemodified inhibitory oligonucleotides efficiently impair TLR7and TLR9-mediated immune responses of human immune cells," PLoS ONE, vol. 10, no. 2, Article ID e0116703, 2015.

[232] C. Weldon, I. Behm-Ansmant, L. H. Hurley et al., "Identification of G-quadruplexes in long functional RNAs using 7deazaguanine RNA," Nature Chemical Biology, vol. 13, no. 1, pp. 18-20, 2017.

[233] R. Kundu, "G-Tetraplex-Induced FRET within Telomeric Repeat Sequences Using PyA-PerA as Energy Donor-Acceptor Pair," Chemistry - An Asian Journal, vol. 11, no. 2, pp. 198-201, 2016.

[234] J. H. Han, N. Chitrapriya, H. S. Lee, Y.-A. Lee, S. K. Kim, and M.-J. Jung, "Behavior of the Guanine Base in G-quadruplexes Probed by the Fluorescent Guanine Analog, 6-Methyl Isoxanthopterin," Bulletin of the Korean Chemical Society, vol. 38, no. 2, pp. 183-190, 2017.

[235] Y. Xu, K. Kaminaga, and M. Komiyama, "G-quadruplex formation by human telomeric repeats-containing RNA in $\mathrm{Na}+$ solution," Journal of the American Chemical Society, vol. 130, no. 33, pp. 11179-11184, 2008.

[236] Y. Xu, T. Ishizuka, T. Kimura, and M. Komiyama, "A U-tetrad stabilizes human telomeric RNA G-quadruplex structure," Journal of the American Chemical Society, vol. 132, no. 21, pp. 72317233, 2010.

[237] R. S. Mendelboum, A. Horváth, J. Aradi et al., "4-Thiodeoxyuridylate-modified thrombin aptamer and its inhibitory effect on fibrin clot formation, platelet aggregation and thrombus growth on subendothelial matrix," Journal of Thrombosis and Haemostasis, vol. 6, no. 10, pp. 1764-1771, 2008.

[238] J. Zhou, K. Murayama, S. Amrane et al., "A "sugar-deficient" Gquadruplex: Incorporation of aTNA in G4 structures," Chemical Science, vol. 4, no. 9, pp. 3693-3698, 2013.

[239] M. Scuotto, E. Rivieccio, A. Varone et al., "Site specific replacements of a single loop nucleoside with a dibenzyl linker may 
switch the activity of TBA from anticoagulant to antiproliferative," Nucleic Acids Research, vol. 43, no. 16, pp. 7702-7716, 2015.

[240] A. D. Gunjal, M. Fernandes, N. Erande, P. R. Rajamohanan, and V. A. Kumar, "Functional isoDNA aptamers: Modified thrombin binding aptamers with a 2/-5/-linked sugar-phosphate backbone (isoTBA)," Chemical Communications, vol. 50, no. 5 , pp. 605-607, 2014.

[241] M. N. Aher, N. D. Erande, M. Fernandes, and V. A. Kumar, "Unimolecular antiparallel G-quadruplex folding topology of 2/-5/-isoTBA sequences remains unaltered by loop composition," Organic and Biomolecular Chemistry, vol. 13, no. 48, pp. 11696-11703, 2015.

[242] B. N. Ames, "Endogenous oxidative DNA damage, aging, and cancer," Free Radical Research, vol. 7, no. 3-6, pp. 121-128, 1989.

[243] A. K. McCullough, M. L. Dodson, and R. S. Lloyd, "Initiation of base excision repair: glycosylase mechanisms and structures," Annual Review of Biochemistry, vol. 68, pp. 255-285, 1999.

[244] D. E. Barnes and T. Lindahl, "Repair and genetic consequences of endogenous DNA base damage in mammalian cells," Annual Review of Genetics, vol. 38, pp. 445-476, 2004.

[245] W. K. Lutz, "Endogenous genotoxic agents and processes as a basis of spontaneous carcinogenesis," Mutation Research/ Reviews in Genetic Toxicology, vol. 238, no. 3, pp. 287-295, 1990.

[246] D. M. Wilson III and V. A. Bohr, "The mechanics of base excision repair, and its relationship to aging and disease," DNA Repair, vol. 6, no. 4, pp. 544-559, 2007.

[247] M. S. Cooke, M. D. Evans, M. Dizdaroglu, and J. Lunec, "Oxidative DNA damage: mechanisms, mutation, and disease," The FASEB Journal, vol. 17, no. 10, pp. 1195-1214, 2003.

[248] H. C. Box, E. E. Budzinski, J. B. Dawidzik, J. S. Gobey, and H. G. Freund, "Free radical-induced tandem base damage in DNA oligomers," Free Radical Biology and Medicine, vol. 23, no. 7, pp. 1021-1030, 1997.

[249] W. Wang, M. Yan, D. Becker, and M. D. Sevilla, "The influence of hydration on the absolute yields of primary free radicals in gamma-irradiated DNA at 77 K. II. Individual radical yields," Radiation Research, vol. 137, no. 1, pp. 2-10, 1994.

[250] J. C. Genereux and J. K. Barton, "Mechanisms for DNA charge transport," Chemical Reviews, vol. 110, no. 3, pp. 1642-1662, 2010.

[251] S. Steenken and S. V. Jovanovic, "How easily oxidizable is DNA? One-electron reduction potentials of adenosine and guanosine radicals in aqueous solution," Journal of the American Chemical Society, vol. 119, no. 3, pp. 617-618, 1997.

[252] J. Sagi, B. Hang, and B. Singer, "Sequence-dependent repair of synthetic AP sites in 15-mer and 35-mer oligonucleotides: Role of thermodynamic stability imposed by neighbor bases," Chemical Research in Toxicology, vol. 12, no. 10, pp. 917-923, 1999.

[253] J. Sagi, A. B. Guliaev, and B. Singer, "15-Mer DNA duplexes containing an abasic site are thermodynamically more stable with adjacent purines than with pyrimidines," Biochemistry, vol. 40, no. 13, pp. 3859-3868, 2001.

[254] J. Sagi, A. Chenna, B. Hang, and B. Singer, "A single cyclic pbenzoquinone adduct can destabilize a DNA oligonucleotide duplex," Chemical Research in Toxicology, vol. 11, no. 4, pp. 329334, 1998.

[255] B. Hang, J. Sagi, and B. Singer, "Correlation between sequencedependent glycosylase repair and the thermal stability of oligonucleotide duplexes containing 1,N6-ethenoadenine," Journal of Biological Chemistry, vol. 273, no. 50, pp. 3340633413, 1998.
[256] J. Sagi and B. Singer, “Thermal destabilization of DNA oligonucleotide duplexes by exocyclic adducts on adenine or cytosine depends on both the base and the size of adduct," IARC Scientific Publications, no. 150, pp. 191-196, 1999.

[257] J. Sagi, A. Perry, B. Hang, and B. Singer, "Differential destabilization of the DNA oligonucleotide double helix by a T.G mismatch, 3,N4-ethenocytosine, 3,N4-ethanocytosine, or an 8-(hydroxymethyl)-3,N4-ethenocytosine adduct incorporated into the same sequence contexts," Chemical Research in Toxicology, vol. 13, no. 9, pp. 839-845, 2000.

[258] A. B. Guliaev, J. Sagi, and B. Singer, "Sequence-dependent conformational perturbation in DNA duplexes containing an $\varepsilon A \cdot T$ mismatch using molecular dynamics simulation," Carcinogenesis, vol. 21, no. 9, pp. 1727-1736, 2000.

[259] L. M. Hellman, T. J. Spear, C. J. Koontz, M. Melikishvili, and M. G. Fried, "Repair of O6-methylguanine adducts in human telomeric G-quadruplex DNA by O6-alkylguanineDNA alkyltransferase," Nucleic Acids Research, vol. 42, no. 15, pp. 9781-9791, 2014.

[260] J. Zhou, M. Liu, A. M. Fleming, C. J. Burrows, and S. S. Wallace, "Neil3 and NEIL1 DNA glycosylases remove oxidative damages from quadruplex DNA and exhibit preferences for lesions in the telomeric sequence context," Journal of Biological Chemistry, vol. 288, no. 38, pp. 27263-27272, 2013.

[261] J. Cadet, T. Douki, D. Gasparutto, and J.-L. Ravanat, “Oxidative damage to DNA: formation, measurement and biochemical features," Mutation Research: Fundamental and Molecular Mechanisms of Mutagenesis, vol. 531, no. 1-2, pp. 5-23, 2003.

[262] P. M. Gannett and T. P. Sura, "Base pairing of 8-oxoguanosine and 8-oxo- $2^{\prime}$-deoxyguanosine with $2^{\prime}$-deoxyadenosine, $2^{\prime}$ deoxycytosine, $2^{\prime}$-deoxyguanosine, and thymidine," Chemical Research in Toxicology, vol. 6, no. 5, pp. 690-700, 1993.

[263] C. J. Burrows and J. G. Muller, "Oxidative nucleobase modifications leading to strand scission," Chemical Reviews, vol. 98, no. 3, pp. 1109-1151, 1998.

[264] W. L. Neeley and J. M. Essigmann, "Mechanisms of formation, genotoxicity, and mutation of guanine oxidation products," Chemical Research in Toxicology, vol. 19, no. 4, pp. 491-505, 2006.

[265] C. M. Gedik and A. Collins, "Establishing the background level of base oxidation in human lymphocyte DNA: results of an interlaboratory validation study," FASEB Journal, vol. 19, no. 1, pp. 82-84, 2005.

[266] D. Mangal, D. Vudathala, J.-H. Park, H. L. Seon, T. M. Penning, and I. A. Blair, "Analysis of 7,8-dihydro-8-oxo-2' -deoxyguanosine in cellular DNA during oxidative stress," Chemical Research in Toxicology, vol. 22, no. 5, pp. 788-797, 2009.

[267] S. Steenken, S. V. Jovanovic, M. Bietti, and K. Bernhard, "The trap depth (in DNA) of 8-oxo-7,8-dihydro-2'deoxyguanosine as derived from electron-transfer equilibria in aqueous solution [2]," Journal of the American Chemical Society, vol. 122, no. 10, pp. 2373-2374, 2000.

[268] W. Luo, J. G. Muller, E. M. Rachlin, and C. J. Burrows, "Characterization of spiroiminodihydantoin as a product of oneelectron oxidation of 8-oxo-7,8-dihydroguanosine," Organic Letters, vol. 2, no. 5, pp. 613-616, 2000.

[269] A. M. Fleming, J. G. Muller, A. C. Dlouhy, and C. J. Burrows, "Structural context effects in the oxidation of 8-Oxo-7,8dihydro-2/- deoxyguanosine to hydantoin products: Electrostatics, base stacking, and base pairing," Journal of the American Chemical Society, vol. 134, no. 36, pp. 15091-15102, 2012. 
[270] S. Oikawa, S. Tada-Oikawa, and S. Kawanishi, "Site-specific DNA damage at the GGG sequence by UVA involves acceleration of telomere shortening," Biochemistry, vol. 40, no. 15, pp. 4763-4768, 2001.

[271] P. L. Opresko, J. Fan, S. Danzy, D. M. Wilson III, and V. A. Bohr, "Oxidative damage in telomeric DNA disrupts recognition by TRF1 and TRF2," Nucleic Acids Research, vol. 33, no. 4, pp. 12301239, 2005.

[272] E. Fouquerel, D. Parikh, and P. Opresko, "DNA damage processing at telomeres: The ends justify the means," DNA Repair, vol. 44, pp. 159-168, 2016.

[273] E. Fouquerel, J. Lormand, A. Bose et al., "Oxidative guanine base damage regulates human telomerase activity," Nature Structural and Molecular Biology, vol. 23, no. 12, pp. 1092-1100, 2016.

[274] Y. Oda, S. Uesugi, M. Ikehara et al., "NMR studies of a DNA containing 8-hydroxydeoxyguanosine," Nucleic Acids Research, vol. 19, no. 7, pp. 1407-1412, 1991.

[275] V. Singh, A. Benz, and J. S. Hartig, "G quadruplexes stabilised by 8-oxo-2l-deoxyguanosine," Chemistry - A European Journal, vol. 17, no. 39, pp. 10838-10843, 2011.

[276] V. A. Szalai, M. J. Singer, and H. H. Thorp, "Site-specific probing of oxidative reactivity and telomerase function using 7,8-dihydro-8-oxoguanine in telomeric DNA," Journal of the American Chemical Society, vol. 124, no. 8, pp. 1625-1631, 2002.

[277] A. Ambrus, D. Chen, J. Dai, T. Bialis, R. A. Jones, and D. Yang, "Human telomeric sequence forms a hybrid-type intramolecular G-quadruplex structure with mixed parallel/antiparallel strands in potassium solution," Nucleic Acids Research, vol. 34, no. 9, pp. 2723-2735, 2006.

[278] D. Renčiuk, I. Kejnovská, P. Školáková, K. Bednářová, J. Motlová, and M. Vorlíčková, "Arrangements of human telomere DNA quadruplex in physiologically relevant $\mathrm{K}+$ solutions," Nucleic Acids Research, vol. 37, no. 19, pp. 6625-6634, 2009.

[279] M. Tomaško, M. Vorlíčková, and J. Sagi, "Substitution of adenine for guanine in the quadruplex-forming human telomere DNA sequence G3(T2AG3)3," Biochimie, vol. 91, no. 2, pp. 171$179,2009$.

[280] J. Sagi, D. Renčiuk, M. Tomaško, and M. Vorlíčková, "Quadruplexes of human telomere DNA analogs designed to contain G:A:G:A, G:G:A:A, and A:A:A:A tetrads," Biopolymers, vol. 93, no. 10, pp. 880-886, 2010.

[281] N. An, A. M. Fleming, and C. J. Burrows, "Human Telomere G-Quadruplexes with Five Repeats Accommodate 8-Oxo-7,8dihydroguanine by Looping out the DNA Damage," ACS Chemical Biology, vol. 11, no. 2, pp. 500-507, 2016.

[282] A. Benz and J. S. Hartig, "Redesigned tetrads with altered hydrogen bonding patterns enable programming of quadruplex topologies," Chemical Communications, no. 34, pp. 4010-4012, 2008.

[283] V. V. Cheong, B. Heddi, C. J. Lech, and A. T. Phan, "Xanthine and 8-oxoguanine in G-quadruplexes: Formation of a G.G.X.O tetrad," Nucleic Acids Research, vol. 43, no. 21, pp. 10506-10514, 2015.

[284] V. V. Cheong, C. J. Lech, B. Heddi, and A. T. Phan, "Inverting the G-Tetrad Polarity of a G-Quadruplex by Using Xanthine and 8Oxoguanine," Angewandte Chemie - International Edition, vol. 55, no. 1, pp. 160-163, 2016.

[285] A. M. Fleming and C. J. Burrows, "G-quadruplex folds of the human telomere sequence alter the site reactivity and reaction pathway of guanine oxidation compared to duplex DNA," Chemical Research in Toxicology, vol. 26, no. 4, pp. 593-607, 2013.
[286] M. Morikawa, K. Kino, T. Oyoshi, M. Suzuki, T. Kobayashi, and H. Miyazawa, "Analysis of guanine oxidation products in double-stranded DNA and proposed guanine oxidation pathways in single-stranded, double-stranded or quadruplex DNA," Biomolecules, vol. 4, no. 1, pp. 140-159, 2014.

[287] X. Li, K. Zheng, Y. Hao, and Z. Tan, "Exceptionally Selective and Tunable Sensing of Guanine Derivatives and Analogues by Structural Complementation in a G-Quadruplex," Angewandte Chemie, vol. 128, no. 44, pp. 13963-13968, 2016.

[288] T. Fujii, P. Podbevšek, J. Plavec, and N. Sugimoto, "Effects of metal ions and cosolutes on G-quadruplex topology," Journal of Inorganic Biochemistry, vol. 166, pp. 190-198, 2017.

[289] A. D. Beniaminov, R. A. Novikov, O. K. Mamaeva et al., "Lightinduced oxidation of the telomeric G4 DNA in complex with $\mathrm{Zn}$ (II) tetracarboxymethyl porphyrin," Nucleic Acids Research, vol. 44, no. 21, pp. 10031-10041, 2016.

[290] V. Esposito, A. Randazzo, A. Virgilio, L. Cozzuto, and L. Mayol, "Effects of a 8-oxoadenosine incorporation on quadruplex structures: Thermal stabilities and structural studies," Nucleosides, Nucleotides and Nucleic Acids, vol. 24, no. 5-7, pp. 783-788, 2005.

[291] L. Petraccone, I. Duro, E. Erra, A. Randazzo, A. Virno, and C. Giancola, "Effect of the incorporation of 2I-deoxy-8(hydroxyl)adenosine on the stability of quadruplexes formed by modified human telomeric DNA," Nucleosides, Nucleotides and Nucleic Acids, vol. 26, no. 6-7, pp. 675-679, 2007.

[292] M. Aggrawal, H. Joo, W. Liu, J. Tsai, and L. Xue, "8-Oxo-7,8dihydrodeoxyadenosine: The first example of a native DNA lesion that stabilizes human telomeric G-quadruplex DNA," Biochemical and Biophysical Research Communications, vol. 421, no. 4, pp. 671-677, 2012.

[293] H. Konvalinová, Z. Dvořáková, D. Renčiuk et al., "Diverse effects of naturally occurring base lesions on the structure and stability of the human telomere DNA quadruplex," Biochimie, vol. 118, article no. 4773, pp. 15-25, 2015.

[294] T. Pfaffeneder, F. Spada, M. Wagner et al., "Tet oxidizes thymine to 5-hydroxymethyluracil in mouse embryonic stem cell DNA," Nature Chemical Biology, vol. 10, no. 7, pp. 574-581, 2014.

[295] N. E. Geacintov and S. Broyde, Introduction and Perspectives on the Chemistry and Biology of DNA Damage, the Chemical Biology of DNA Damage, Wiley-VCH Verlag GmbH \& Co. $\mathrm{KGaA}, 2010$.

[296] L. Petraccone, E. Erra, L. Nasti et al., "H-NMR study of the quadruplex [d(TGGGT)]4 containing a modified thymine," Nucleosides, Nucleotides and Nucleic Acids, vol. 22, no. 5-8, pp. 1677-1680, 2003.

[297] A. Virgilio, L. Petraccone, M. Scuotto et al., "5-hydroxymethyl2 -deoxyuridine residues in the thrombin binding aptamer: investigating anticoagulant activity by making a tiny chemical modification," ChemBioChem, vol. 15, no. 16, pp. 2427-2434, 2014.

[298] A. Virgilio, V. Esposito, L. Mayol, C. Giancola, L. Petraccone, and A. Galeone, "The oxidative damage to the human telomere: Effects of 5-hydroxymethyl-2/-deoxyuridine on telomeric Gquadruplex structures," Organic and Biomolecular Chemistry, vol. 13, no. 27, pp. 7421-7429, 2015.

[299] Z. Wang, "DNA damage and mutagenesis," in Molecular and Biochemical Toxicology, R. C. Smart and E. Hodgson, Eds., pp. 441-491, Wiley, Hoboken, NJ, USA, 2008.

[300] J. Völker, G. E. Plum, H. H. Klump, and K. J. Breslauer, "DNA repair and DNA triplet repeat expansion: The impact 
of abasic lesions on triplet repeat DNA energetics," Journal of the American Chemical Society, vol. 131, no. 26, pp. 9354-9360, 2009.

[301] J. Völker, G. Eric Plum, H. H. Klump, and K. J. Breslauer, "Energy crosstalk between DNA lesions: Implications for allosteric coupling of DNA repair and triplet repeat expansion pathways," Journal of the American Chemical Society, vol. 132, no. 12, pp. 4095-4097, 2010.

[302] V. Esposito, L. Martino, G. Citarella et al., "Effects of abasic sites on structural, thermodynamic and kinetic properties of quadruplex structures," Nucleic Acids Research, vol. 38, no. 6, Article ID gkp1087, pp. 2069-2080, 2009.

[303] P. Školáková, K. Bednářová, M. Vorlíčková, and J. Sagi, "Quadruplexes of human telomere dG3(TTAG3)3 sequences containing guanine abasic sites," Biochemical and Biophysical Research Communications, vol. 399, no. 2, pp. 203-208, 2010.

[304] T. Fujimoto, S.-I. Nakano, D. Miyoshi, and N. Sugimoto, "The effects of molecular crowding on the structure and stability of G-quadruplexes with an abasic site," Journal of Nucleic Acids, vol. 2011, Article ID 857149, 2011.

[305] A. Virgilio, L. Petraccone, V. Esposito, G. Citarella, C. Giancola, and A. Galeone, "The abasic site lesions in the human telomeric sequence d[TA(G 3T2A)3G3]: A thermodynamic point of view," Biochimica et Biophysica Acta - General Subjects, vol. 1820, no. 12, pp. 2037-2043, 2012.

[306] M. Cevec and J. Plavec, "Role of loop residues and cations on the formation and stability of dimeric DNA G-quadruplexes," Biochemistry, vol. 44, no. 46, pp. 15238-15246, 2005.

[307] S. Nagatoishi and N. Sugimoto, "Interaction of water with the G-quadruplex loop contributes to the binding energy of Gquadruplex to protein," Molecular BioSystems, vol. 8, no. 10, pp. 2766-2770, 2012.

[308] M. Babinský, R. Fiala, I. Kejnovská et al., "Loss of loop adenines alters human telomere d(AG(3)(TTAG(3))(3)) quadruplex folding," Nucleic Acids Research, vol. 42, no. 22, pp. 14031-14041, 2014.

[309] M. Vorlíčková, I. Kejnovská, J. Sagi et al., "Circular dichroism and guanine quadruplexes," Methods, vol. 57, no. 1, pp. 64-75, 2012.

[310] R. M. Abu-Ghazalah, S. Rutledge, L. W. Y. Lau, D. N. Dubins, R. B. MacGregor, and A. S. Helmy, "Concentration-dependent structural transitions of human telomeric DNA sequences," Biochemistry, vol. 51, no. 37, pp. 7357-7366, 2012.

[311] J. Palacký, M. Vorlíčková, I. Kejnovská, and P. Mojzeš, "Polymorphism of human telomeric quadruplex structure controlled by DNA concentration: A Raman study," Nucleic Acids Research, vol. 41, no. 2, pp. 1005-1016, 2013.

[312] I. Kejnovska, M. Vorlickova, M. Brazdova, and J. Sagi, "Stability of human telomere quadruplexes at high DNA concentrations," Biopolymers, vol. 101, no. 4, pp. 428-438, 2014.

[313] Y. Y. Li, R. Abu-Ghazalah, B. Zamiri, and R. B. Macgregor, "Concentration-dependent conformational changes in GQforming ODNs," Biophysical Chemistry, vol. 211, pp. 70-75, 2016.

[314] T. Wu, M. Ye, T. Mao et al., "Human telomeric hybrid-2-overhybrid-1 G-quadruplex targeting and a selective hypersalinetolerant sensor using abasic site-engineered monomorphism," Analytica Chimica Acta, vol. 964, pp. 161-169, 2017.

[315] V. Esposito, L. Pirone, L. Mayol, E. Pedone, A. Virgilio, and A. Galeone, "Exploring the binding of $\mathrm{d}(\mathrm{GGGT}) 4$ to the HIV-1 integrase: An approach to investigate G-quadruplex aptamer/target protein interactions," Biochimie, vol. 127, pp. 1922, 2016.
[316] A. V. Sekridova, A. M. Varizhuk, and G. E. Pozmogova, "The impact of non-nucleotide insertions on thermal stability of DNA G-quadruplexes," International Research Journal, vol. 5, pp. 116-118, 2016.

[317] B. Heddi, N. Martín-Pintado, Z. Serimbetov, T. M. A. Kari, and A. T. Phan, "G-quadruplexes with $(4 n-1)$ guanines in the G-tetrad core: Formation of a G-triad-water complex and implication for small-molecule binding," Nucleic Acids Research, vol. 44, no. 2, pp. 910-916, 2016.

[318] V. T. Mukundan and A. T. Phan, "Bulges in G-quadruplexes: Broadening the definition of G-quadruplex-forming sequences," Journal of the American Chemical Society, vol. 135, no. 13, pp. 5017-5028, 2013.

[319] B. Demple, A. Jacobsson, M. Olsson, P. Robins, and T. Lindahl, "Repair of alkylated DNA in Escherichia coli. Physical properties of O 6-methylguanine-DNA methyltransferase," Journal of Biological Chemistry, vol. 257, no. 22, pp. 13776-13780, 1982.

[320] G. Koike, H. Maki, H. Takeya, H. Hayakawa, and M. Sekiguchi, "Purification, structure, and biochemical properties of human O6-methylguanine-DNA methyltransferase," Journal of Biological Chemistry, vol. 265, no. 25, pp. 14754-14762, 1990.

[321] C. S. Mekmaysy, L. Petraccone, N. C. Garbett et al., "Effect of O6-methylguanine on the stability of G-quadruplex DNA," Journal of the American Chemical Society, vol. 130, no. 21, pp. 6710-6711, 2008.

[322] M. Ikeda, M. Kamimura, Y. Hayakawa, A. Shibata, and Y. Kitade, "Cover Picture: Reduction-Responsive Guanine Incorporated into G-Quadruplex-Forming DNA (ChemBioChem 14/2016)," ChemBioChem, vol. 17, no. 14, p. 1291, 2016.

[323] Y. Zheng, C. Lorenzo, and P. A. Beal, "DNA editing in DNA/RNA hybrids by adenosine deaminases that act on RNA," Nucleic Acids Research, p. gkx050.

[324] F. W. Smith and J. Feigon, "Strand orientation in the DNA quadruplex formed from the oxytricha telomere repeat oligonucleotide d(G4T4G4) in solution," Biochemistry, vol. 32, no. 33, pp. 8682-8692, 1993.

[325] M. Kinoshita, S. Takaya, T. Shibata, H. Hemmi, and Y. Yamamoto, "NMR detection and characterization of I-quartets in parallel DNA quadruplexes," Chemistry Letters, vol. 44, no. 8, pp. 1107-1109, 2015.

[326] A. Risitano and K. R. Fox, "Inosine substitutions demonstrate that intramolecular DNA quadruplexes adopt different conformations in the presence of sodium and potassium," Bioorganic and Medicinal Chemistry Letters, vol. 15, no. 8, pp. 2047-2050, 2005.

[327] L. Hu, K. W. Lim, S. Bouaziz, and A. T. Phan, "Giardia telomeric sequence D(TAGGG) 4 forms two intramolecular Gquadruplexes in $\mathrm{K}+$ solution: Effect of loop length and sequence on the folding topology," Journal of the American Chemical Society, vol. 131, no. 46, pp. 16824-16831, 2009.

[328] A. T. Phan, V. Kuryavyi, H. Y. Gaw, and D. J. Patel, "Smallmolecule interaction with a five-guanine-tract g-quadruplex structure from the human myc promoter," Nature Chemical Biology, vol. 1, no. 3, pp. 167-173, 2005.

[329] N. Q. Do, K. W. Lim, M. H. Teo, B. Heddi, and A. T. Phan, "Stacking of G-quadruplexes: NMR structure of a Grich oligonucleotide with potential anti-HIV and anticancer activity," Nucleic Acids Research, vol. 39, no. 21, pp. 9448-9457, 2011.

[330] K. W. Lim, L. Lacroix, D. J. E. Yue, J. K. C. Lim, J. M. W. Lim, and A. T. Phan, "Coexistence of two distinct G-quadruplex 
conformations in the hTERT promoter," Journal of the American Chemical Society, vol. 132, no. 35, pp. 12331-12342, 2010.

[331] A. Tanaka, J. Choi, and T. Majima, "Folding and structural polymorphism of G-quadruplex formed from a long telomeric sequence containing six GGG tracts," RSC Advances, vol. 4, no. 103, pp. 59071-59077, 2014.

[332] Z. Zhang, J. Dai, E. Veliath, R. A. Jones, and D. Yang, "Structure of a two-G-tetrad intramolecular G-quadruplex formed by variant human telomeric sequence in $\mathrm{K}+$ solution: Insights into the interconversion of human telomeric G-quadruplex structures," Nucleic Acids Research, vol. 38, no. 3, Article ID gkp1029, pp. 1009-1021, 2009.

[333] R. W. Harkness and A. K. Mittermaier, "G-register exchange dynamics in guanine quadruplexes," Nucleic Acids Research, vol. 44, no. 8, pp. 3481-3494, 2016.

[334] S. H. Jeon, J. Moon, M. W. Lee, and S. K. Kim, "Effects of guanine bases at the central loop on stabilization of the quadruplex DNAs and their interactions with Meso-tetrakis(Nmethylpyridium-4-yl)porphyrin," Biophysical Chemistry, vol. 205, pp. 9-15, 2015.

[335] X. Fan, L. Sun, Y. Wu, L. Zhang, and Z. Yang, "Bioactivity of 2/deoxyinosine-incorporated aptamer AS1411," Scientific Reports, vol. 6, Article ID 25799, 2016.

[336] J. Novotný, P. Kulhánek, and R. Marek, "Biocompatible xanthine-quadruplex scaffold for ion-transporting DNA channels," Journal of Physical Chemistry Letters, vol. 3, no. 13, pp. 1788-1792, 2012.

[337] J. Novotný, Y. P. Yurenko, P. Kulhánek, and R. Marek, “Tailoring the properties of quadruplex nucleobases for biological and nanomaterial applications," Physical Chemistry Chemical Physics, vol. 16, no. 29, pp. 15241-15248, 2014.

[338] Y. P. Yurenko, J. Novotný, V. Sklenář, and R. Marek, "Exploring non-covalent interactions in guanine- and xanthine-based model DNA quadruplex structures: a comprehensive quantum chemical approach," Physical Chemistry Chemical Physics, vol. 16, no. 5, pp. 2072-2084, 2014.

[339] J. E. Smith, C. Lu, and J.-S. Taylor, "Effect of sequence and metal ions on UVB-induced anti cyclobutane pyrimidine dimer formation in human telomeric DNA sequences," Nucleic Acids Research, vol. 42, no. 8, pp. 5007-5019, 2014.

[340] M. Gulston, C. de Lara, T. Jenner, E. Davis, and P. O’Neill, "Processing of clustered DNA damage generates additional double-strand breaks in mammalian cells post-irradiation," Nucleic Acids Research, vol. 32, no. 4, pp. 1602-1609, 2004.

[341] T. J. Jenner, J. Fulford, and P. O’Neill, “Contribution of base lesions to radiation-induced clustered DNA damage: Implication for models of radiation response," Radiation Research, vol. 156, no. 5, pp. 590-593, 2001.

[342] I. Kejnovská, K. Bednářová, D. Renčiuk et al., "Clustered abasic lesions profoundly change the structure and stability of human telomeric G-quadruplexes," Nucleic Acids Research, vol. 45, no. 8, pp. 4294-4305, 2017.

[343] K. D. Robertson, "DNA methylation and human disease," Nature Reviews Genetics, vol. 6, no. 8, pp. 597-610, 2005.

[344] A. Bird, "DNA methylation patterns and epigenetic memory," Genes \& Development, vol. 16, no. 1, pp. 6-21, 2002.

[345] M. François, W. Leifert, R. Tellam, and M. Fenech, "Gquadruplexes: A possible epigenetic target for nutrition," Mutation Research - Reviews in Mutation Research, vol. 764, pp. 101107, 2015.
[346] F. Eckhardt, J. Lewin, R. Cortese et al., "DNA methylation profiling of human chromosomes 6, 20 and 22," Nature Genetics, vol. 38, no. 12, pp. 1378-1385, 2006.

[347] S. C. Wu and Y. Zhang, "Active DNA demethylation: many roads lead to Rome," Nature Reviews Molecular Cell Biology, vol. 11, no. 9, pp. 607-620, 2010.

[348] M. Bachman, S. Uribe-Lewis, X. Yang et al., "5-Formylcytosine can be a stable DNA modification in mammals," Nature Chemical Biology, vol. 11, no. 8, pp. 555-557, 2015.

[349] C.-X. Song, C. Yi, and C. He, "Mapping recently identified nucleotide variants in the genome and transcriptome," Nature Biotechnology, vol. 30, no. 11, pp. 1107-1116, 2012.

[350] M. Wagner, J. Steinbacher, T. F. J. Kraus et al., "Age-Dependent Levels of 5-Methyl-, 5-Hydroxymethyl-, and 5-Formylcytosine in Human and Mouse Brain Tissues," Angewandte Chemie International Edition, vol. 54, no. 42, pp. 12511-12514, 2015.

[351] J. Lin, J.-Q. Hou, H.-D. Xiang et al., "Stabilization of Gquadruplex DNA by C-5-methyl-cytosine in bcl-2 promoter: implications for epigenetic regulation," Biochemical and Biophysical Research Communications, vol. 433, no. 4, pp. 368-373, 2013.

[352] M. M. Molnar, R. K. Morgan, B. Summerford, T. A. Brooks, and R. M. Wadkins, "Epigenetic modification effects on the i-motif and G-quadruplex forms of single strand DNA," in Proceeding of the 68th Southeastern Regional Meeting of the American Chemical Society, SERMACS-78, Columbia, SC, USA, October 23-26, 2016.

[353] B. Zamiri, M. Mirceta, K. Bomsztyk, R. B. Macgregor, and C. E. Pearson, "Quadruplex formation by both G-rich and C-rich DNA strands of the C9orf72 (GGGGCC)8•(GGCCCC)8 repeat: Effect of CpG methylation," Nucleic Acids Research, vol. 43, no. 20, pp. 10055-10064, 2015.

[354] A. M. Fleming, Y. Ding, and C. J. Burrows, "Oxidative DNA damage is epigenetic by regulating gene transcription via base excision repair," Proceedings of the National Academy of Sciences of the United States of America, vol. 114, no. 10, pp. 2604-2609, 2017.

[355] A. M. Fleming and C. J. Burrows, "8-Oxo-7,8-dihydroguanine, friend and foe: Epigenetic-like regulator versus initiator of mutagenesis," DNA Repair, vol. 56, pp. 75-83, 2017. 

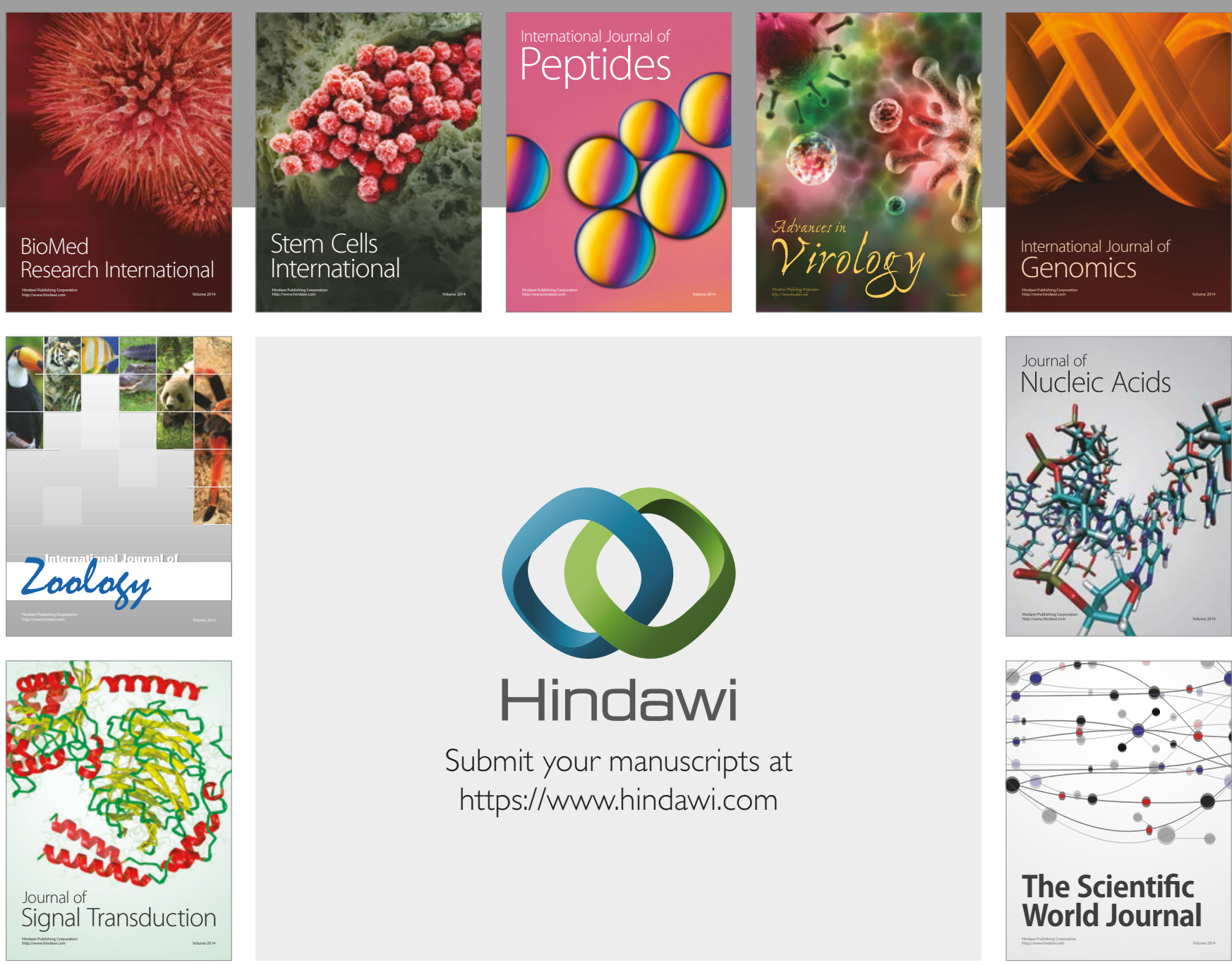

Submit your manuscripts at

https://www.hindawi.com
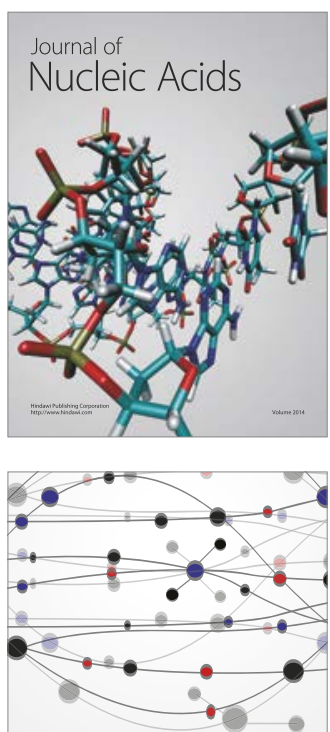

The Scientific World Journal

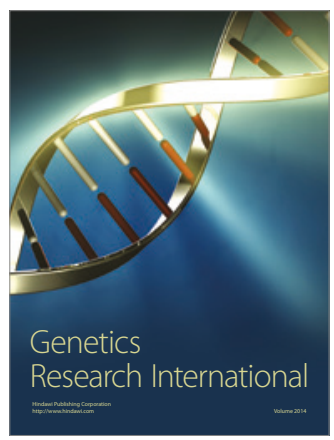

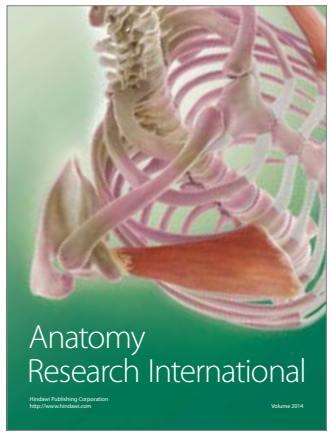

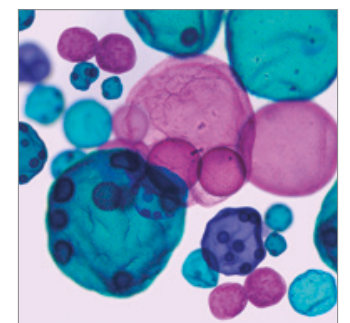

International Journal of Microbiology
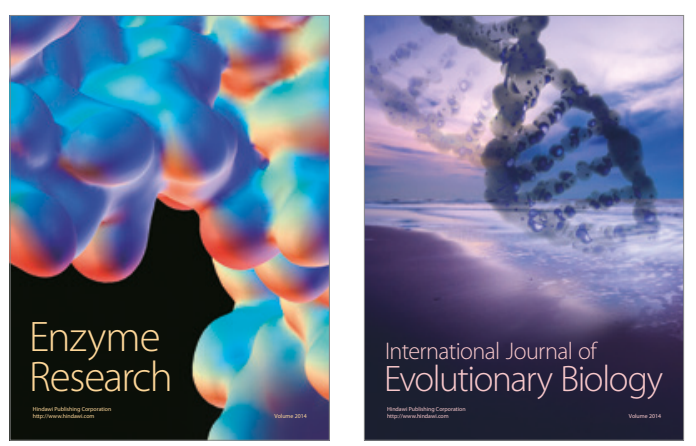
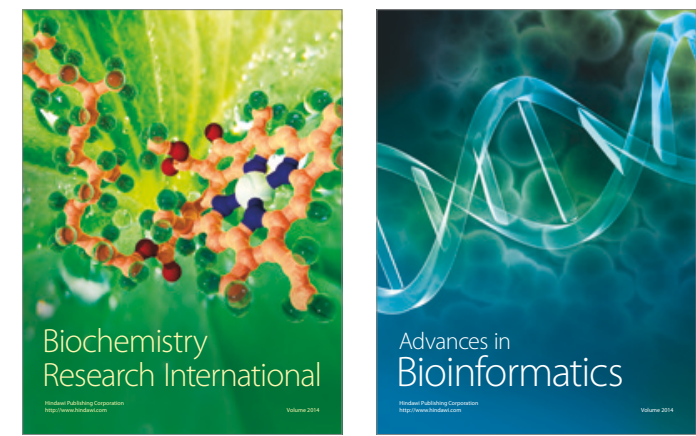

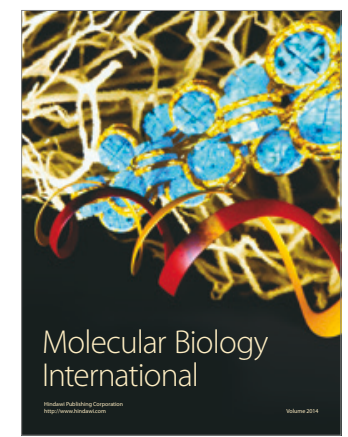

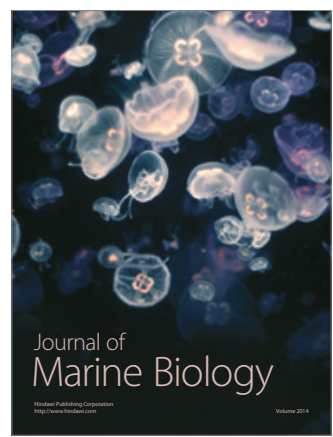

